\title{
质子耦合电子转移在有机合成中的应用
}

\author{
周子杰 ${ }^{a}$ 孔祥梅 $a$ 刘天飞*,a,b \\ ( ${ }^{a}$ 南开大学元素有机化学国家重点实验室 天津 300071) \\ ${ }^{b}$ 中国科学院上海有机化学研究所有机氟化学重点实验室 上海 200032)
}

\begin{abstract}
摘要 质子耦合电子转移(PCET)是一类非传统的氧化还原反应, 因其电子和质子转移过程具有独特的相互依赖性, 这 类反应表现出特殊的反应性和选择性. PCET 反应在包括天然和人工系统的氧化还原过程中, 如小分子活化等, 起到了 相当关键的作用. 最近, PCET 反应在有机合成中的应用引起了广泛的关注和兴趣. 人们实现了通过 PCET 机理的 X-H 键(如 $\mathrm{C}-\mathrm{H}$ 键、 $\mathrm{N}-\mathrm{H}$ 键、 $\mathrm{S}-\mathrm{H}$ 键和 $\mathrm{O}-\mathrm{H}$ 键等)的活化，基于该过程合成了多种重要结构和基础骨架、获得了不同的 合成砌块和天然产物. 此外, 通过 PCET 反应机理, 人们不仅获得了 $\mathrm{C}=\mathrm{Y}$ 键等多重键的还原产物, 而且获得了底物之 间的偶联产物. 综述了近年来 PCET 在有机合成中的应用和发展, 并且对该领域未来的发展进行了展望.
\end{abstract}

关键词 质子耦合电子转移; 有机合成; 自由基反应; 官能化

\section{Applications of Proton-Coupled Electron Transfer in Organic Synthesis}

\author{
Zhou, Zijie $^{a} \quad$ Kong, Xiangmei ${ }^{a} \quad$ Liu, Tianfei ${ }^{*, a, b}$ \\ ( ${ }^{a}$ State Key Laboratory of Elemento-organic Chemistry, Nankai University, Tianjin 300071) \\ $\left({ }^{b}\right.$ Key Laboratory of Organofluorine Chemistry, Shanghai Institute of Organic Chemistry, \\ Chinese Academy of Sciences, Shanghai 200032)
}

\begin{abstract}
Proton-coupled electron transfer (PCET) reactions are a kind of unconventional redox reactions, which exhibit special reactivities and selectivities due to their unique interdependent electron-proton transfer mechanisms. There are three possible pathways of PCET processes, including stepwise electron transfer followed by proton transfer (ETPT), proton transfer followed by electron transfer (PTET), and concerted pathway in which electron and proton transfer synchronously (CEPT), avoiding intermediates with high energy. These reactions have been playing a key role in numerous areas in organic chemistry, inorganic chemistry, bioorganic chemistry, organometallic and material chemistry, including the redox processes in natural and artificial systems, such as the activation for small molecules. Recently, the application of PCET reactions in organic synthesis has received a great deal of attentions and interests. Being accompanied by the development of electrochemical methods and photocatalysts, more and more novel reactions in electrochemistry and photochemistry involve PCET processes have been reported. Applying these electrochemical and photochemical methods, the activation of $\mathrm{X}-\mathrm{H}$ bond has been achieved via PCET processes, including $\mathrm{C}-\mathrm{H}$ bond, $\mathrm{N}-\mathrm{H}$ bond, $\mathrm{P}-\mathrm{H}$ bond, $\mathrm{S}-\mathrm{H}$ bond or $\mathrm{O}-\mathrm{H}$ bond. Thus, based on these crucial processes, a number of vital structures and fundamental frameworks can be synthesized, and various synthetic building blocks and natural products have been attained. For example, pharmaceutical building blocks like $2^{\circ}$-piperidines can be cyanated at their $\alpha$-position; substituted dimeric pyrroloindolines such as $(-)$-calycanthidine, $(-)$-chimonanthine, and ( - )-psychotriasine have also been successfully synthesized via PCET mechanism. Moreover, not only the products of reduction of multiple bonds $(\mathrm{C}=$ $\mathrm{Y}$ bond such as $\mathrm{C}=\mathrm{C}$ bond, $\mathrm{C}=\mathrm{N}$ bond and $\mathrm{C}=\mathrm{O}$ bond), but also the products of self/cross-coupling have been achieved via PCET mechanism. In this review, the recent applications and developments of PCET mechanism in organic synthesis are summarized, including new catalyst systems and new reagents, especially with electrochemical and photochemical methodologies. The future of this area has also been demonstrated from both experimental and theoretical aspects.
\end{abstract}

Keywords proton-coupled electron transfer; organic synthesis; radical reactions; functionalization

\footnotetext{
* Corresponding author. E-mail: tianfeiliu@nankai.edu.cn

Received June 1, 2021; revised July 22, 2021; published online August 24, 2021.

Dedicated to the 100th anniversary of Chemistry at Nankai University.

Project supported by the Startup Fund from Nankai University.

南开大学人事人才支持资助项目.
} 


\section{Introduction}

Proton-coupled electron transfer (PCET) reactions, in which an electron coupled with a proton transfer from donors to acceptors in a stepwise or concerted way, ${ }^{[1-6]}$ are a sort of fundamental reactions in a various of areas, including organic chemistry, ${ }^{[7-9]}$ inorganic chemistry, ${ }^{[10-11]}$ bioorganic chemistry, ${ }^{[12]}$ organometallic and material chemistry $^{[6,13-19]}$, and so on. Most recently, the applications of PCET in organic synthesis have attracted attentions from organic chemists. Since this process was first discovered experimentally in $1981,{ }^{[20]}$ this area has been developing flourishingly accompanied by the inventions of new electrochemical and photochemical methods. ${ }^{[21-24]}$ Nowadays, more and more new methodologies involving PCET mechanism have been reported (Figure 1), including two main types of the bond transformation. First of all, via PCET processes, the homolysis of $\mathrm{X}-\mathrm{H}$ bond for constructing a diverse range of structures has been well developed, including $\mathrm{C}-\mathrm{H}\left(\Delta H^{\circ}=414 \mathrm{~kJ} / \mathrm{mol}\right), \mathrm{N}-\mathrm{H}$ $\left(\Delta H^{\circ}=389 \mathrm{~kJ} / \mathrm{mol}\right), \mathrm{O}-\mathrm{H}\left(\Delta H^{\circ}=464 \mathrm{~kJ} / \mathrm{mol}\right), \mathrm{P}-\mathrm{H}$ $\left(\Delta H^{\circ}=322 \mathrm{~kJ} / \mathrm{mol}\right)$ and $\mathrm{S}-\mathrm{H}\left(\Delta H^{\circ}=338 \mathrm{~kJ} / \mathrm{mol}\right)$ bonds, ${ }^{[25-26]}$ some of which are commonly considered as strong bonds, while PCET provides some favorable pathways for their activations. Secondly, the reduction of double bonds via $\mathrm{PCET}$ has been reported, including $\mathrm{C}=\mathrm{C}, \mathrm{C}=\mathrm{N}$ and $\mathrm{C}=\mathrm{O}$ bonds, to form not only the hydrogenated products, but also advanced structures via the $\mathrm{C}-\mathrm{C}$ bond formation after the generation of radical intermediates. In these reactions, neither sensitive reductants like metal hydrides nor dihydrogen need to be used, and the conditions are always very mild. With these two types of reactions, a number of vital structures and fundamental frameworks can be synthesized, and various synthetic building blocks and natural products have been attained.

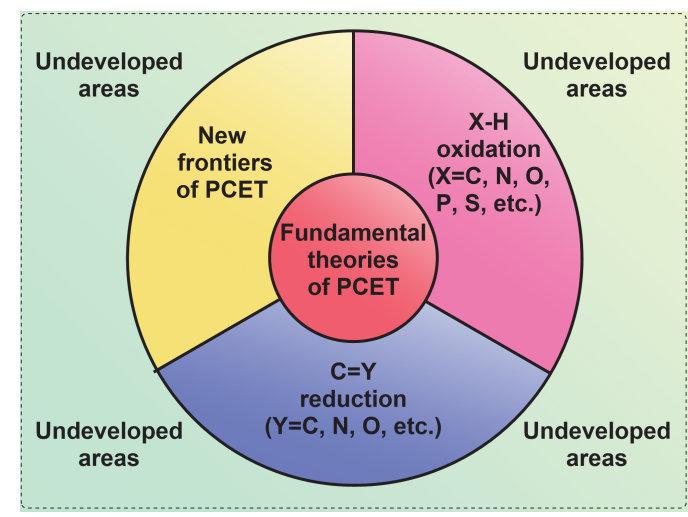

Figure 1 Synthetic application of PCET

The structure of this review is as follows: at the beginning of this review, we introduce the elementary principles of PCET in a concise and most understandable way to organic chemists, avoiding deriving complicated formulas of physical chemistry. Then, we summarize two main types of PCET reactions in organic synthesis which we mentioned above. At the end of this review, we prospect the frontiers of
PCET in organic synthesis, and discuss how the new existing PCET mechanisms like inner-sphere PCET and excited-state PCET (ES-PCET) would influence the development of PCET theories and their applications in organic synthesis in future.

\section{Elementary principles of PCET for or- ganic chemists}

Both electron and proton transfer theory have existed for a long time, ${ }^{[27-31]}$ and the coupled electron and proton transfer has been largely developed, ${ }^{[32-34]}$ and mathematical functions on PCET have been well summarized. ${ }^{[35-37]}$ In this chapter, we hope to introduce the elementary principles of PCET to organic chemists in a concise but practical way, for helping them explore and develop new organic synthetic methodologies using this process in the future. Herein, we focus on some concepts which might confuse them for a while, such as the differences and relations between HAT and PCET process (Figure 2).

HAT was once considered as a sub-class of PCET. ${ }^{[24]}$ Although some attempts were made to unify the PCET, HAT and other analogous reactions, ${ }^{[39]}$ because of the differences between them, distinguishing HAT processes from PCET processes is of great significance to PCET's applications, ${ }^{[6]}$ especially for organic chemists. In an HAT process both proton and electron transfer from a single donor to a single acceptor adiabatically, sharing the starting and the final orbitals, the most classic example is the benzyl/toluene self-exchange reaction. ${ }^{[13,34,42]}$ The driving force of HAT reaction is commonly discussed as the difference in the bond dissociation free energies (BDFEs) of the reactants and products. Comparatively, in PCET reactions the proton and the electron move non-adiabatically. ${ }^{[34]}$ We can classify the PCET reactions into two types: in the first type, both proton and electron transfer from a single donor to a single acceptor, but they go to different sets of orbitals, just as the PCET reaction between phenoxyl radical and phenol; and the second type can be called multisite-PCET (MS-PCET), including both oxidative process and reductive process, where the proton and the electron can transfer from the same donor to two different acceptors in the former oxidative process, or they can transmit from different donors to a same acceptor in the later reductive process. The differences between HAT process and the above mentioned PCET processes are concisely illustrated in Figure 2, and the effective bond dissociation free energies $\left(\mathrm{BDFE}_{\mathrm{eff}}\right)$ can be introduced to thermodynamically describe the driving force of PCET reaction. The free energy relationship between the rate constants and $\mathrm{BDFE}_{\text {eff }}$ have been applied to reveal the detailed mechanism in several PCET processes. ${ }^{[6,13-14,38-39,43-44]}$ Examples of HAT and PCET reactions are shown in Scheme 1.

A PCET mechanism may undergo three pathways (Figure 3), separately named ETPT, in which electron transfers first and later proton transfers stepwisely; PTET, in which 

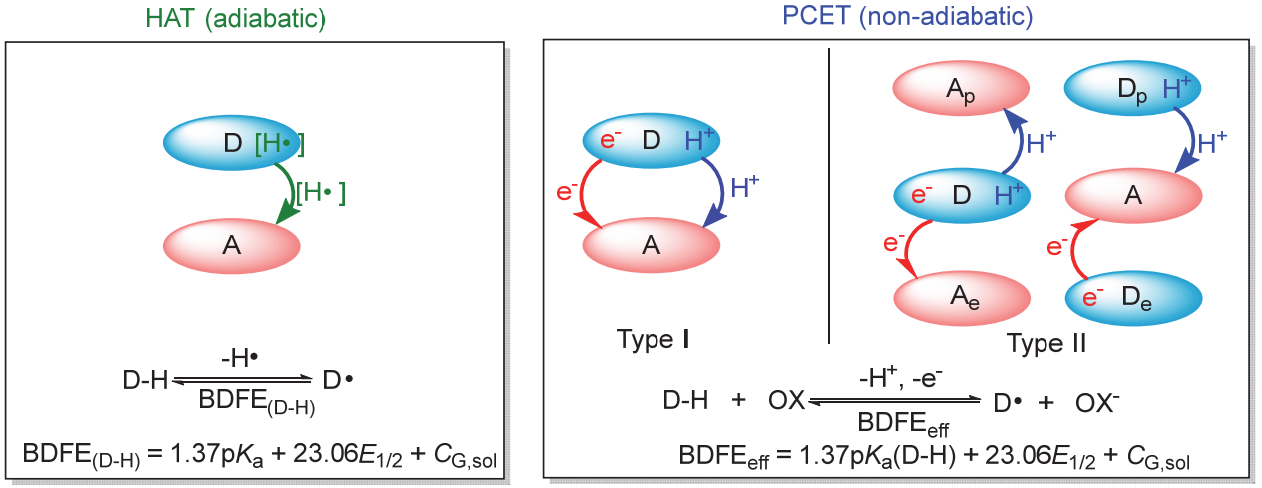

Figure 2 Concise illustration of HAT process with its BDFE equation (left), and illustrations of PCET processes with the corresponding effective BDFE equation (right) ${ }^{[38-39]}$

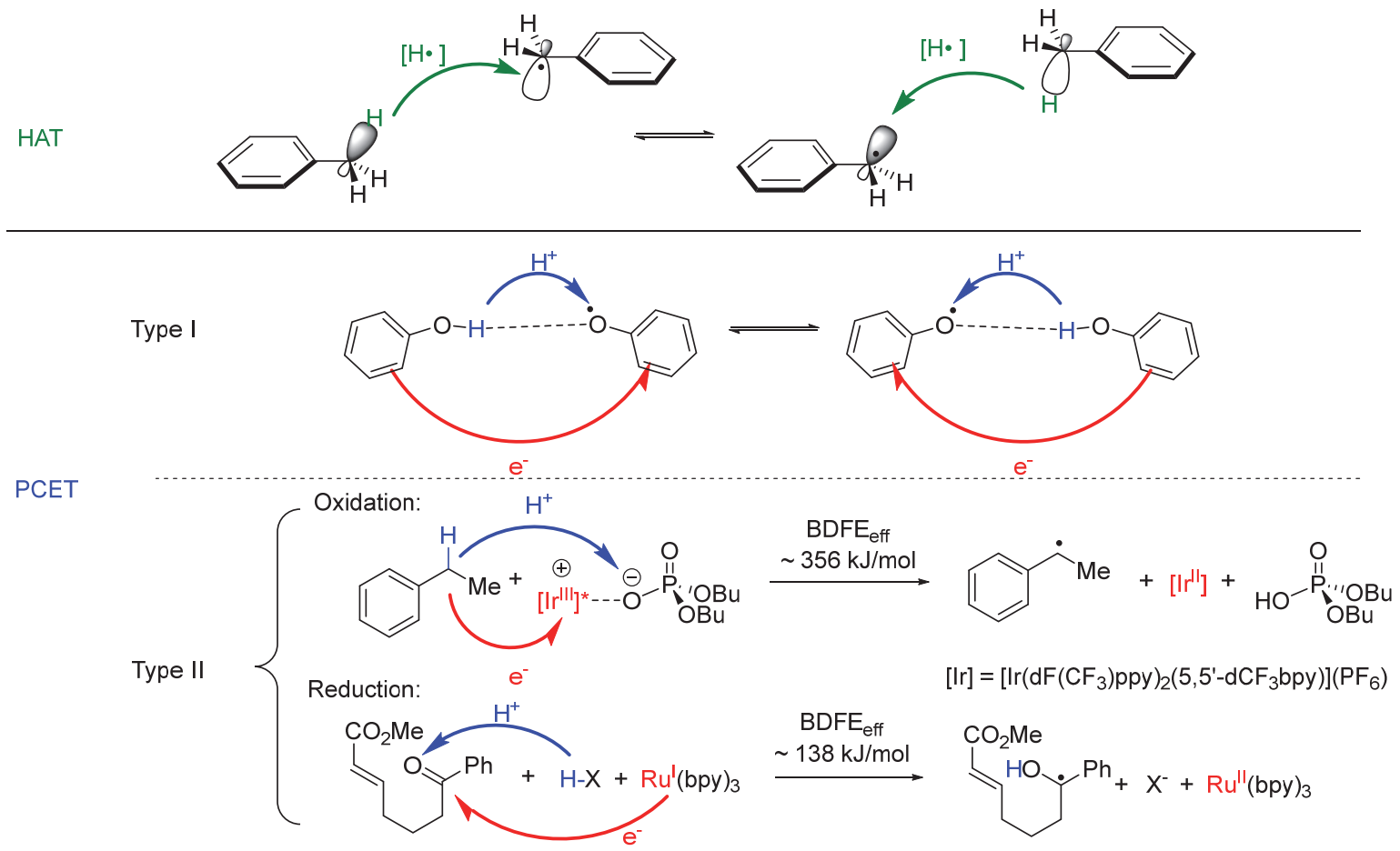

Scheme 1 Reactions via HAT and two types of PCET mechanism ${ }^{[34,40-41]}$

proton transfers first and then electron transfers stepwisely; CEPT (concerted electron proton transfer), in which electron and proton transfer synchronously. Recently, a series of publications have experimentally revealed the fundamental rules on how to switch the mechanism among these three by controlling the driving force and other conditions of the reaction. ${ }^{[6,13-14]}$ These guidelines and rules may guide the organic chemists to design new redox reactions more rationally and efficiently, to make the reaction conditions much milder and greener in future. As shown in Figure 3, the first two pathways are sequential and generate distinct but short-lived intermediates. While for reactions proceed by CEPT mechanism, the electron and proton transfer in one step via a single transition state. Compared to stepwise PCET processes, it is obvious that the CEPT processes avoid the formation of the high-energy intermediates, which

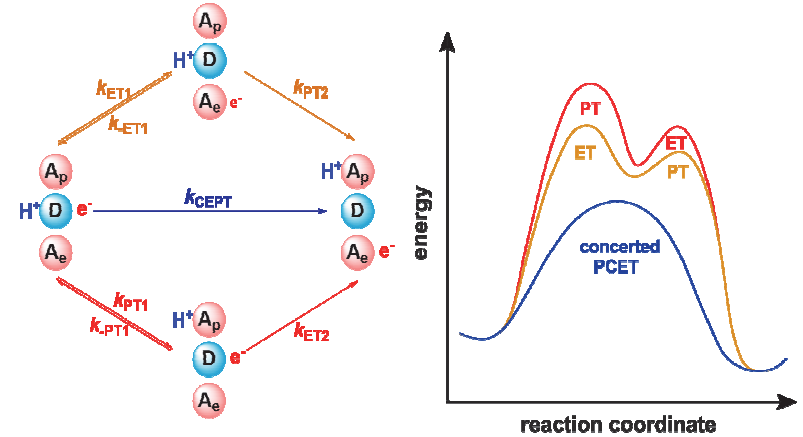

Figure 3 Three pathways of PCET mechanism (left) ${ }^{[6,13-14,45-46]}$ and their corresponding reaction coordinates (right, redrawn from Acc Chem Res. 2016, 49(8), 1546 1556. $)^{[7]}$

should benefit the whole reaction kinetically. In the fol- 
lowing sections, the successful examples of PCET processes applied in both oxidative and reductive organic reactions are discussed in details.

\section{Oxidation of $\mathrm{X}-\mathrm{H}$ bond}

\section{$3.1 \mathrm{C}-\mathrm{H}$ bond}

Numerous works on the functionalization of unactivated $\mathrm{C}\left(\mathrm{sp}^{3}\right)-\mathrm{H}$ bond have been reported. Some of the most famous ones are from Yu's group, they reported a series of transition metal-catalyzed $\mathrm{C}-\mathrm{H}$ activation reactions including palladium(II)-catalyzed $\mathrm{C}-\mathrm{H}$ activation; ${ }^{[47-48]}$ they also developed methods for $\mathrm{C}-\mathrm{H}$ cleavage via weak coordination. ${ }^{[49]}$ Shi's group ${ }^{[50]}$ and other groups also reported different metal-catalyzed $\mathrm{C}-\mathrm{H}$ activation methods including iron-catalyzed reactions. Recently, the activation of $\mathrm{C}-\mathrm{H}$ bond enabled by PCET mechanism was also developed, including $\mathrm{C}-\mathrm{H}$ oxidation, nitration, alkylation, cyanation, and so on. ${ }^{[40,45,51-65]}$

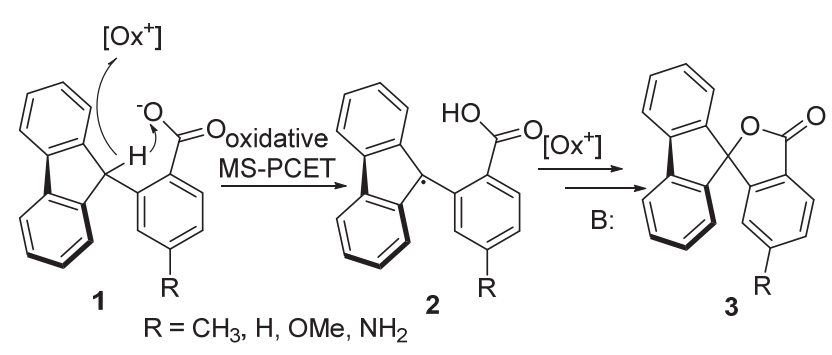

Scheme 2 C - H oxidation via MS-PCET mechanism

The mechanism of PCET-promoted $\mathrm{C}-\mathrm{H}$ activation was first studied in detail by Mayer and his collaborators. Since 2018, his group has published papers on a novel strategy for the cleavage of $\mathrm{C}-\mathrm{H}$ bond through MS-PCET in the presence of base and redox cofactors (Scheme 2). ${ }^{[51]}$ Their study suggests that this reaction can proceed under mild conditions with low driving forces, which means PCET mechanism would be applicable for the oxidation of strong $\mathrm{C}-\mathrm{H}$ bond. The theoretical study of this CEPT $\mathrm{C}-\mathrm{H}$ bond activation was published by Hammes-Schiffer's group in the same year. ${ }^{[52]}$ Their computed results are qualitatively consistent with Mayer's experimental results. Specifically, by modeling the same $\mathrm{C}-\mathrm{H}$ oxidation reaction with a vibronically nonadiabatic PCET theory, Hammes-Schiffer and co-workers got the slope of $\lg k_{\mathrm{PCET}}$, the logarithm of the reaction rate constant, versus $\lg K$, the logarithm of the equilibrium constant, which is 0.37 , while the experimental result in Mayer's work is 0.21. Both are less than 0.5, which is predicted by semiclassical Marcus-theory type treatments. Accordingly, they draw the conclusion that the experimental results can be reproduced qualitatively with this PCET model, and the relatively shallow slope can be explained by the negative reaction free energies associated with the dominant pairs of vibronic states. Further, Mayer et $a l{ }^{[45]}$ extend this system to different substituted fluorenyl-benzoates and various outer-sphere oxidants. Through kinetic analysis, it is concluded that the second- order rate constant is more dependent on the $\mathrm{p} K_{\mathrm{a}}$ of the internal base than on the character of the outer-sphere oxidant, which suggests that the transition states of these reactions are imbalanced. Based on these experiments, Hammes-Schiffer et al. ${ }^{[53]}$ explained the reason by using vibronically nonadiabatic PCET theory: the enhancement of internal carboxylate's basicity increases the driving force of proton transfer on the one hand and decreases the equilibrium proton donor-acceptor distance on the other hand.

In terms of organic synthesis methodology, in 2017, Ooi and co-workers ${ }^{[54]}$ reported the homo-dimerization of 3-aryl oxindoles via PCET pathways, with their newly developed intramolecular ion-pairing acridinium phenoxide framework (6) as a chemical redox catalyst that was used for intramolecular ion-pairing electron-transfer (Scheme 3). This redox catalyst could coordinate with the substrate through oxygen anion in hydrogen bond, followed by the generation of substrate radicals via PCET mechanism.
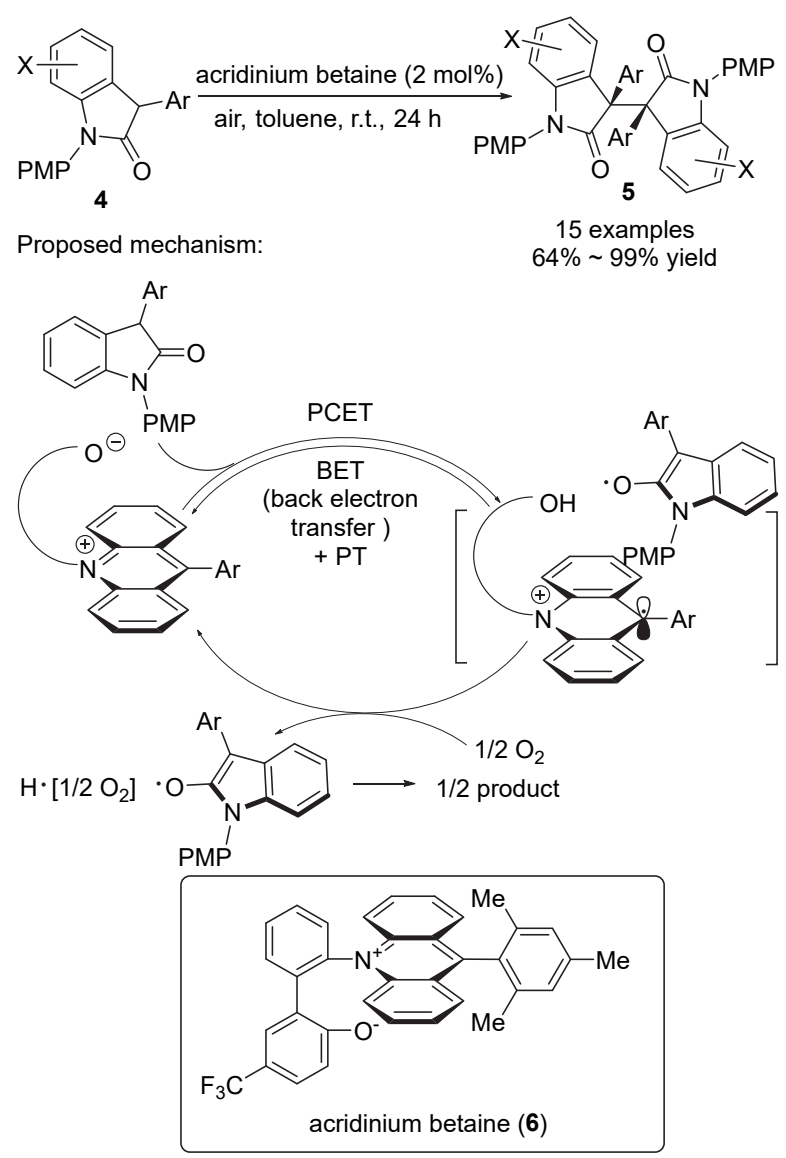

Scheme 3 Homo-dimerization of 3-aryl oxindoles via PCET pathways

In 2018, List's group ${ }^{[55]}$ reported a direct and asymmetric $\alpha$-aryloxylation of cyclic ketones in the presence of quinones (Scheme 4). For simple aldehydes and ketones, their organocatalytic enatioselective $\alpha$-functionalizations can be achieved by a well-established single-electron oxidation of enamines, while the oxidation of $\alpha$-substituted ketones hasn't been reported. The authors introduced enol catalysis 
to the $\alpha$-branched ketones oxidation. With 1,1'-binaphthol (BINOL) derived chiral phosphate (C1) as catalyst, $\alpha$-substituted ketones could generate the higher $\alpha, \alpha$-substituted products with 1,4-benzoquinone in both good yields and enantioselectivities. This is the first example for the direct, catalytic, and asymmetric $\alpha$-aryloxylation of cyclic $\alpha$-branched ketones, and it could be a reference for other enantioselective transformations involving enol derived radical intermediates.

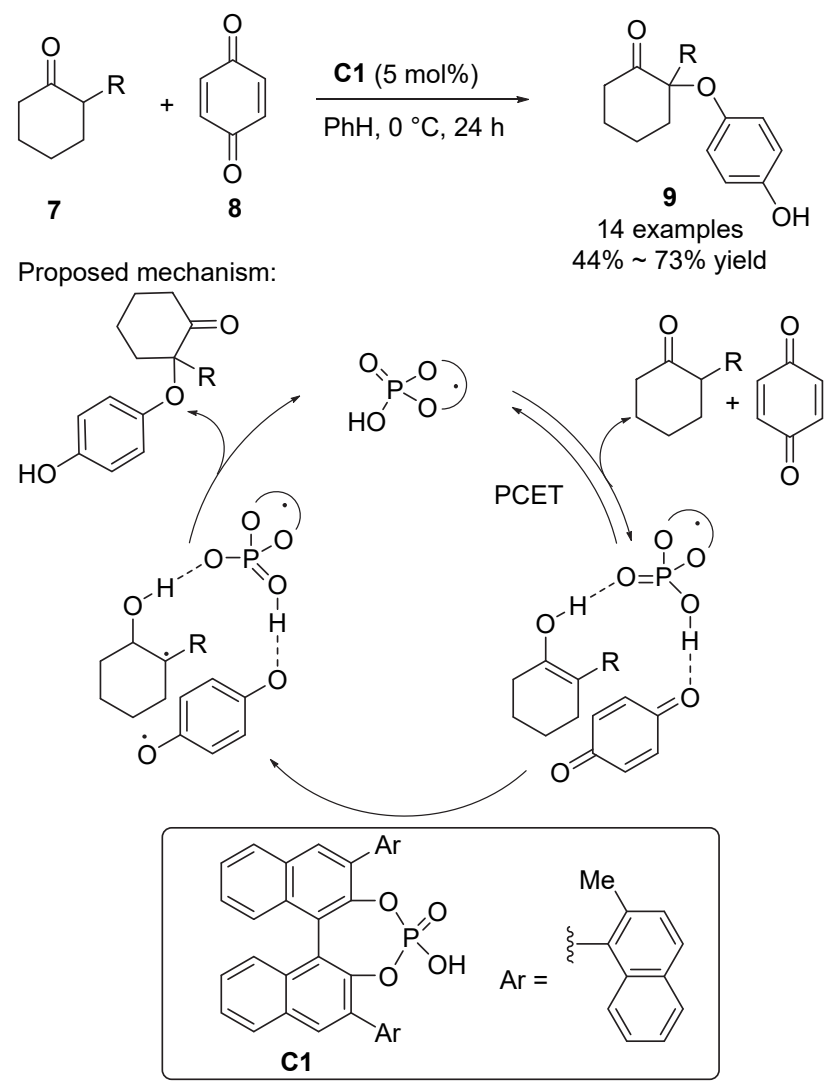

Scheme 4 Asymmetric $\alpha$-aryloxylation of cyclic ketones in the presence of quinones

Besides, $\mathrm{C}-\mathrm{H}$ oxidation can be achieved via photochemical ways. In 2019, Alexanian and co-workers ${ }^{[40]}$ reported a visible-light-induced catalytic method for intermolecular $\mathrm{C}-\mathrm{H}$ alkylation of unactivated aliphatic $\mathrm{C}\left(\mathrm{sp}^{3}\right)-\mathrm{H}$ bond through PCET under mild conditions, with 1,1-bis(phenylsulfonyl)ethylene (11) as the alkene partner (Scheme 5). Their further study demonstrated a large scope of both hydrocarbon substrate and alkene partner of this method. By applying PCET mechanism, their strategy enabled the cleavage of $\mathrm{C}-\mathrm{H}$ bond without highly reactive heteroatom-centered radicals, which are typically required in related photoredox catalyst functionalizations.

In 2020, Ready's group ${ }^{[56]}$ published a paper of a photocatalytic method for the C-alkylation of amines with alkyl bromides (Scheme 6). Although $\alpha$-branched amines are important and fundamental building blocks for the organic synthesis, their traditional synthesis strategies, such as nucleophilic addition, require highly reactive organometallic reagents and rigorous conditions. While with PC1 as the

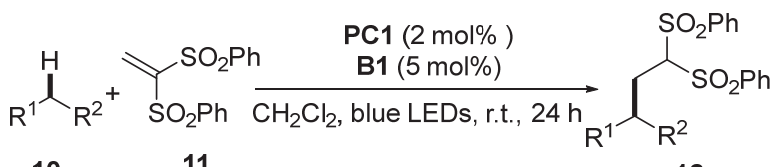

$$
\begin{aligned}
& 10 \quad 11 \\
& 12 \\
& 21 \text { examples }
\end{aligned}
$$
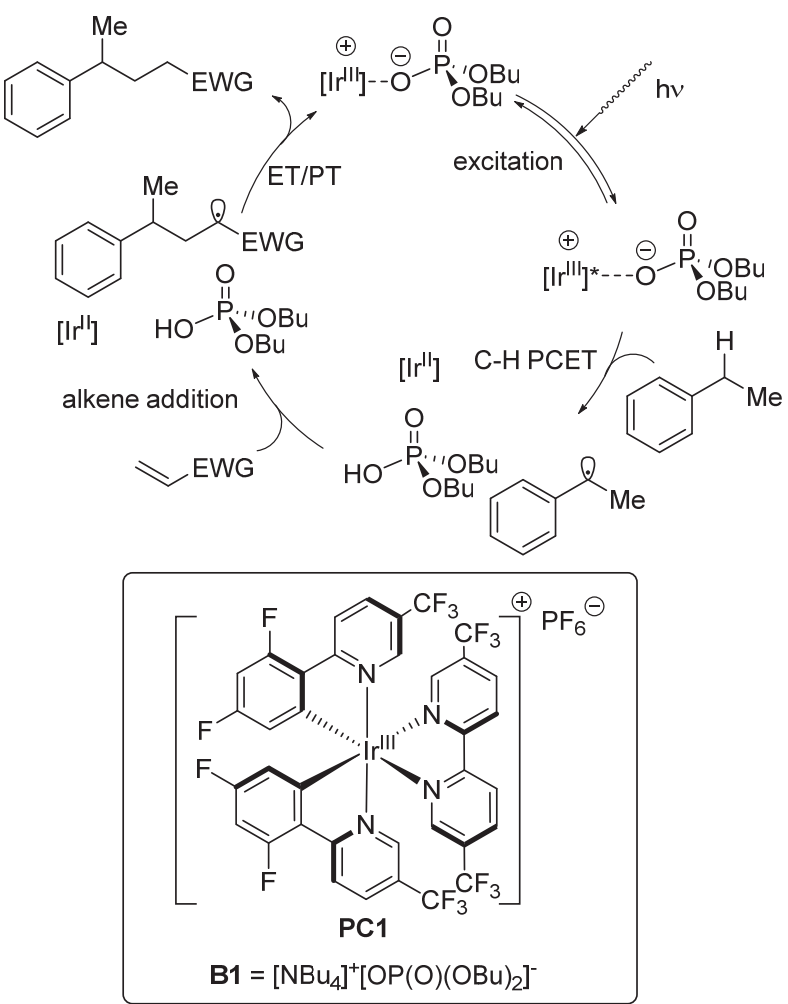

Scheme 5 Intermolecular $\mathrm{C}-\mathrm{H}$ alkylation of unactivated aliphatic $\mathrm{C}\left(\mathrm{sp}^{3}\right)-\mathrm{H}$ bond

photocatalyst and $\mathrm{Na}_{2} \mathrm{CO}_{3}$ as mild base, this newly developed method could proceed in a mild and atom-economical way at room temperature. Further exploration also showed a broad substrates scope. The reaction pathway involves the initial photocatalytic generation of an $\alpha$-amino radical via a PCET process, followed by a radical recombination and then achieve the final product.

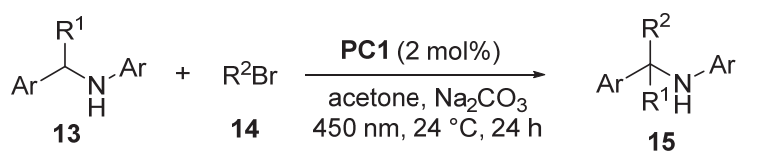

79 examples $27 \% \sim 85 \%$ yield
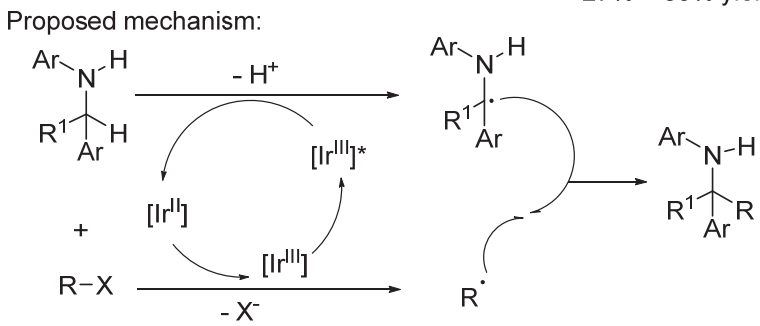

Scheme 6 C-alkylation of amines with alkyl bromides 
As for electrochemical methods, in 2017, Xu's group ${ }^{[57]}$ reported the cross-dehydrogenative coupling of $\mathrm{C}\left(\mathrm{sp}^{3}\right)-\mathrm{H}$ and $\mathrm{C}\left(\mathrm{sp}^{2}\right)-\mathrm{H}$ bond from malonate amides, accessing the synthesis of C3-fluorinated oxindoles via an electrochemical oxidation reaction pathway (Scheme 7). These compounds are important organic molecules for their applications in pharmaceutical and agrochemical industries. This method was found to have a good functional group tolerance. In the reaction pathway, the starting material was oxidized by dicyclopentadienyl iron(III) cation after a rate-limiting deprotonation step, followed by annulation and subsequent electron and proton transfer steps. His group presumed that this could possibly be a PCET proceed. This method leads to an effective, highly chemoselective and straightforward way for the synthesis of organofluorine compounds.

In 2018, Stahl and co-workers ${ }^{[58]}$ developed an effective method for electrochemical $\alpha$-cyanation of second piperidines, which are a kind of ideal pharmaceutical building blocks, without requiring protection of the $\mathrm{N}-\mathrm{H}$ bond (Scheme 8). In this method, they employed 9-azabicyclo- nonane $N$-oxyl (ABNO) as the catalytic mediator that promotes the dehydrogenation of second piperidines to generate the cyclic imine. This highly effective, as well as user-friendly method, is appropriate for substrates with a large range of heterocyclic substituents, and can achieve the synthesis of a various of unnatural amino acids.

An example of electrochemical method using manganese salts as redox catalyst was also reported. In the same year, Mo and co-workers ${ }^{[59]}$ developed a Mn-mediated electrochemical synthesis of azaheterocycles via sequential trifluoromethylation/C( $\left.\mathrm{sp}^{2}\right)-\mathrm{H}$ functionalization (Scheme 9). This method achieved the introduction of trifluoromethyl group into organic molecules, and a large range of functionalized heterocycles with a $\mathrm{CF}_{3}$ group including trifluoromethylated oxindoles could be accessed via this method.

Similar to the work of Ooi's group, in 2020, Paul and co-workers ${ }^{[60]}$ also developed a homo-coupling reaction route that accesses the dimerization of 3-substituted 2oxindoles under a mild electrochemical condition (Scheme 10). They explored the reaction under conditions with and

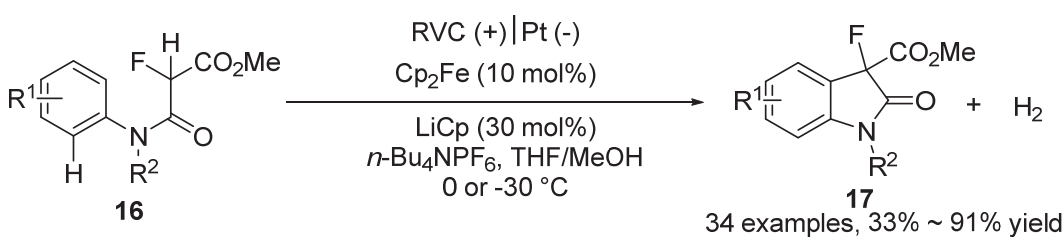

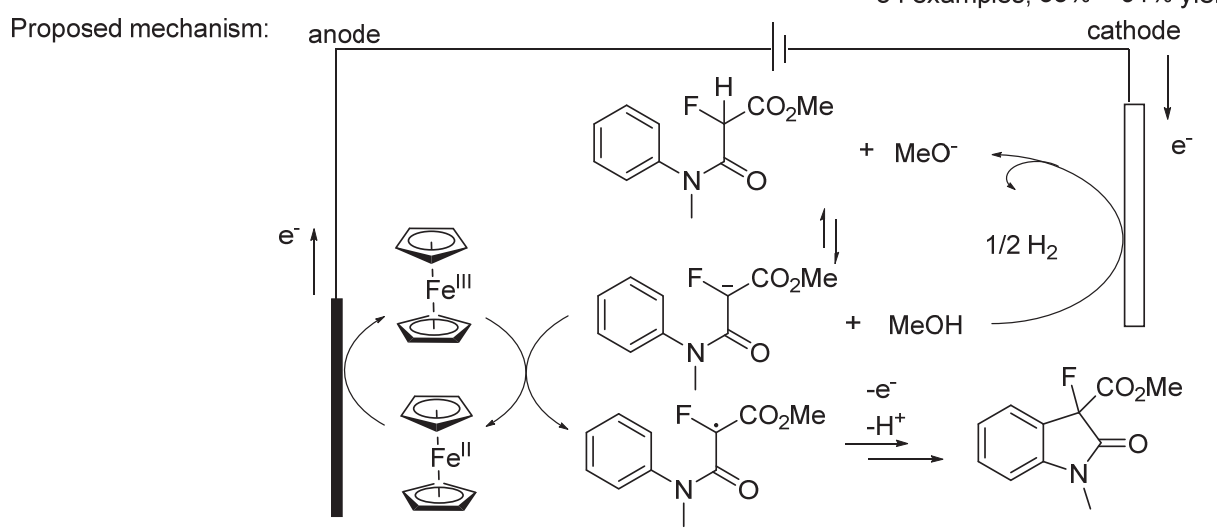

Scheme 7 Cross-dehydrogenative coupling of $\mathrm{C}\left(\mathrm{sp}^{3}\right)-\mathrm{H}$ and $\mathrm{C}\left(\mathrm{sp}^{2}\right)-\mathrm{H}$ bonds

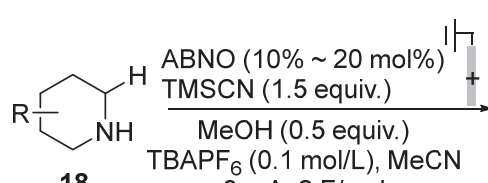

18

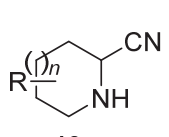

19
(1) Remove electrolyte (2) $\mathrm{TsOH}$

(3) wash

(3) wash 30 examples $26 \% \sim 87 \%$ yield

Proposed mechanism:

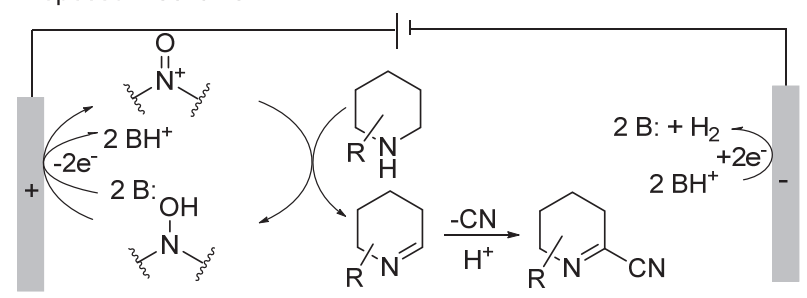

Scheme 8 Electrochemical $\alpha$-cyanation of second piperidines and synthesis of amino acids 


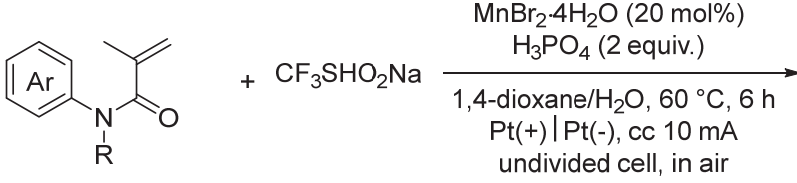

22a<smiles>[R]C(=O)N(C(=O)C(=C)C(=O)OCC)c1ccccc1</smiles>

22b<smiles>C=C(C)C(=O)N(C)C(=O)c1ccccc1</smiles><smiles>[R20]N1C(=O)[C@](C)(CC(F)(F)F)c2ccccc21</smiles>

15 examples, $30 \% \sim 79 \%$ yield<smiles>[R]C(=O)N1C(=O)[C@@](CC(F)(F)F)(C(=O)OCC)c2ccccc21</smiles>

23b

8 examples, $59 \% \sim 76 \%$ yield<smiles>CN1C(=O)c2ccccc2[C@@](C)(CC(F)(F)F)C1=O</smiles>

8 examples, $41 \% \sim 83 \%$ yield

Proposed mechanism:

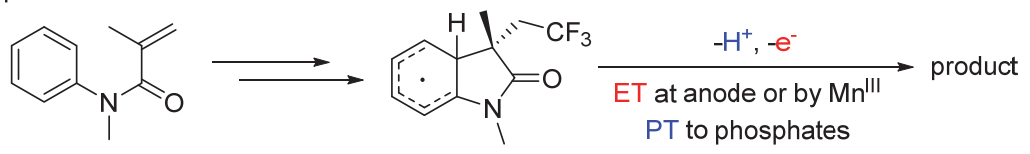

Scheme 9 Mn-mediated electrochemical synthesis of azaheterocycles<smiles>[R]C1C(=O)Nc2cc[R1]([H])cc21</smiles>

24

Proposed mechanism:

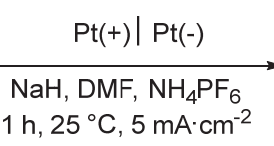

h, $25^{\circ} \mathrm{C}, 5 \mathrm{~mA} \cdot \mathrm{cm}^{-2}$

$\mathrm{R}^{3}$

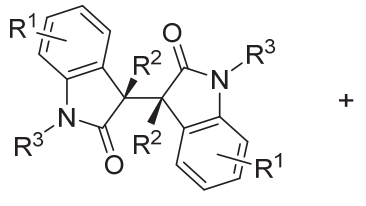

major 25a

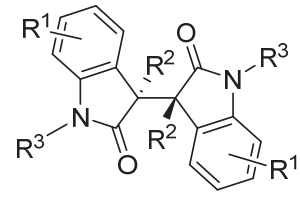

minor $25 b$

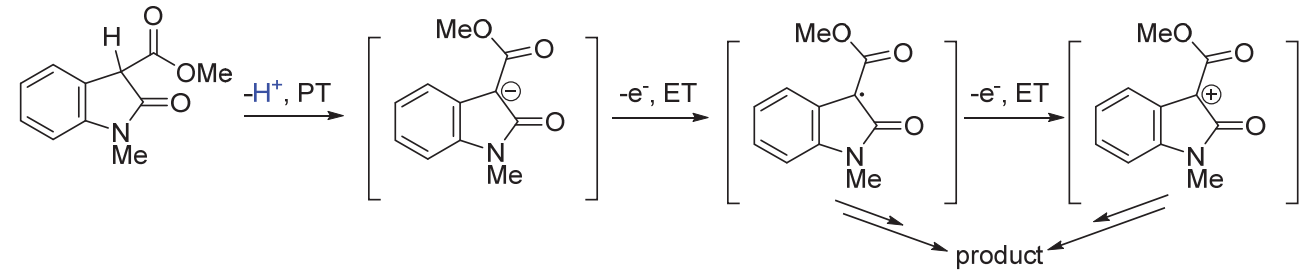

Scheme 10 Dimerization of 3-substituted 2-oxindoles

without addition of base. According to their investigation towards the mechanism of both methods, it was suggested that the base-assisted reaction follows a stepwise PT-ET-ET pathway, while an ET-PT-ET pathway for the other one. Further exploration showed that an appropriate choice of base with proper concentration can accelerate the reaction rate through PCET mechanism because of the remarkable decrease in the kinetic barrier. Sulekha Sharma's work brought an effective electro-oxidative synthesis for a large scope of structurally diverse dimeric 2-oxindoles with vicinal quaternary centers.

In the same year, Pan and co-workers ${ }^{[61]}$ reported an electrochemical synthesis of 1-naphthols from alkynes and 1,3-dicarbonyl compounds via an intermolecular annulation reaction, in which dicyclopentadienyl iron was used as redox catalyst (Scheme 11). This novel strategy leads to the synthesis of important polysubstituted naphthalene compounds under an environmentally friendly and efficient condition.

Notably, in 2019, Ritter's group ${ }^{[62]}$ reported a method for the synthesis of benzylic alcohols by selective monooxidation of alkylated benzenes, in which bis(methanesulfonyl) peroxide severs as an oxidant (Scheme 12). Their method achieved the conversion of benzylic $\mathrm{C}-\mathrm{H}$ bond to benzylic alcohols, and they proposed that the reaction proceeds via a PCET pathway. With copper(I) acetate as catalyst, and the presence of peroxide MsOOMs and DMSOAc, they obtained benzylic mesylate intermediates, which were then converted to the alcohol products in the mixture of hexafluoroisopropanol (HFIP) and water. This catalytic intermolecular alkylation can functionalize a diverse range of small molecules efficiently under mild reaction conditions, 


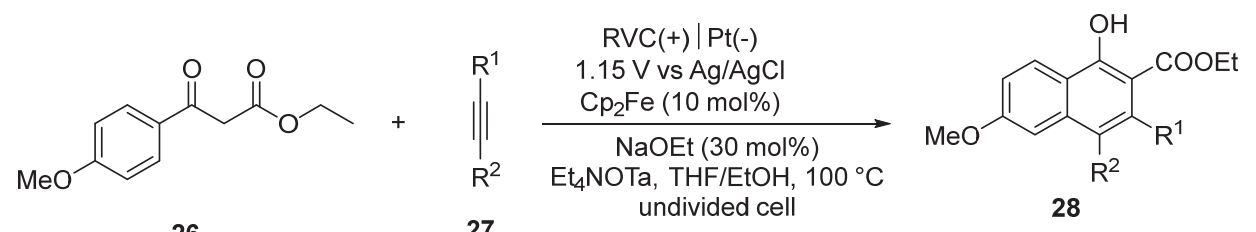

26

27 undivided cell

30 examples, $27 \% \sim 78 \%$ yield

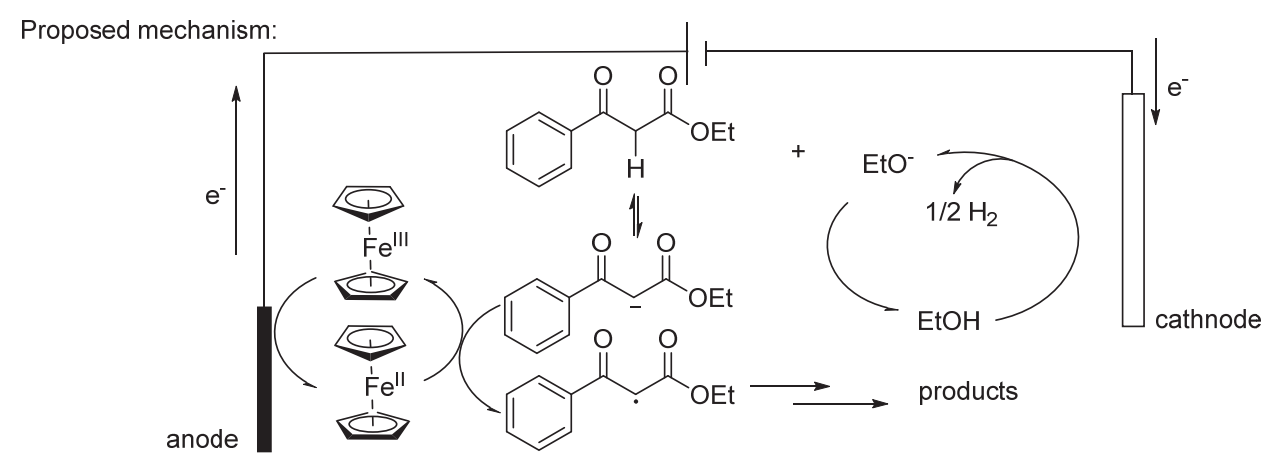

Scheme 11 Synthesis of 1-naphthols from alkynes and 1,3-dicarbonyl compounds<smiles>[R][R]1ccccc1C([R])[18F]</smiles>

29
$\stackrel{\mathrm{HFIP} / \mathrm{H}_{2} \mathrm{O}(\mathrm{V}: \mathrm{V}=1: 1,0.2 \mathrm{~mol} / \mathrm{L})}{1 \sim 12 \mathrm{~h}, 23^{\circ} \mathrm{C}}$

30<smiles>[R1]C(O)c1[R]ccc1</smiles>

31

Proposed mechanism:

21 examples, $30 \% \sim 91 \%$ yield

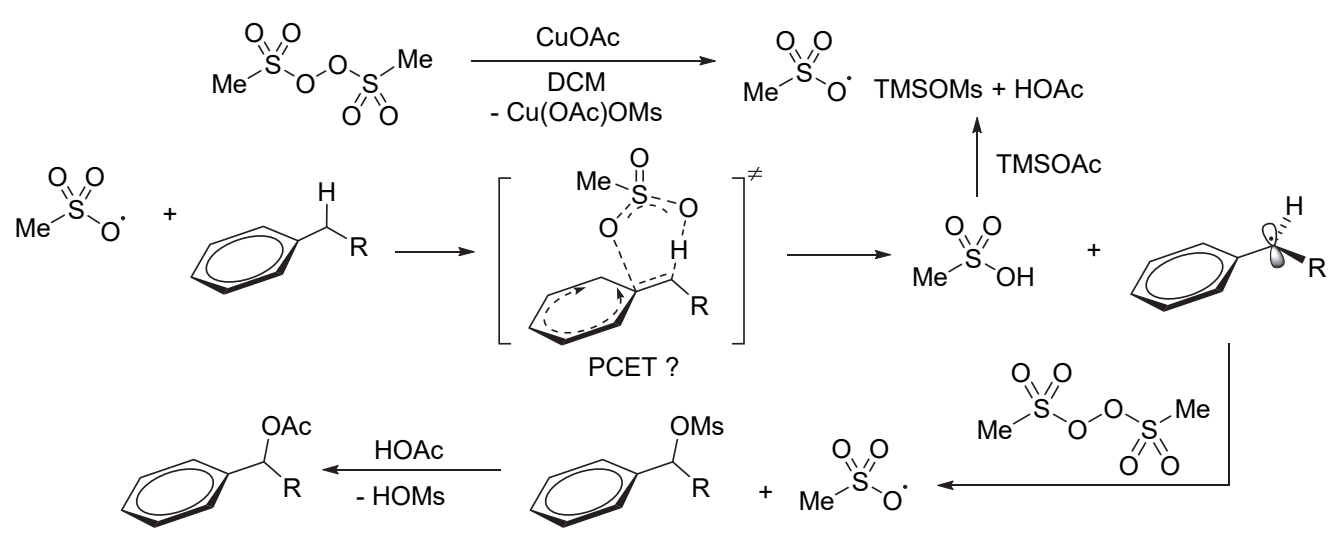

Scheme 12 Synthesis of benzylic alcohols by selective monooxidation of alkylated benzenes

and may set up a unique and general approach to $\mathrm{C}-\mathrm{H}$ alkylations. In addition, compared to traditional functionalizations involving photoredox catalysis, $\mathrm{C}-\mathrm{H}$ cleavage promoted by PCET mechanism avoids the employment of highly reactive heteroatom-centered radicals. This method could be of great use for the development of drugs and agrochemicals. However, it should be noted that the possibility of a HAT mechanism-enabled $\mathrm{C}-\mathrm{H}$ cleavage can not be ruled out completely in this case. ${ }^{[6,34,42]}$

In 2019, Huang and co-workers ${ }^{[63]}$ reported a visiblelight-induced reductive Minisci reaction for $N$-heterocyclic aromatics with aldehydes under mild conditions (Scheme 13). This method leads to the formation of a diverse range of functionalized $N$-heterocyclic aromatics including alkylated and benzylated nitrogen heteroarenes. Notably, aldehydes are served as alkylation reagents as well as reductants.

In 2021, He and co-workers ${ }^{[64]}$ reported the electrochemical multicomponent synthesis of a wide range of 4-selanylpyrazoles in generally good yields (Scheme 14). The employments of electrochemical method and radical reactions avoid the requirement of heating conditions, transition-metal catalysts and chemical oxidants. More importantly, this method features a high functional group tolerance and scalability due to the mild reaction conditions.

\section{$3.2 \mathrm{~N}-\mathrm{H}$ bond}

The cleavage of $\mathrm{N}-\mathrm{H}$ bond might be one of the most common applications of PCET in organic synthesis. For example, $N$-centered radicals generated from the breaking of $\mathrm{N}-\mathrm{H}$ bond could be important intermediates in the 
formation of heterocycles structures, which are commonly found in a variety of natural products. Moreover, PCET mechanism also contributes to some unusual chemoselectivities, for example, there are several methods for the activation of relatively strong amide $\mathrm{N}-\mathrm{H}$ bond in compounds which consist of weaker allylic $\mathrm{C}-\mathrm{H}$ bond. ${ }^{[66]}$ Thus, reactions involving the cleavage of $\mathrm{N}-\mathrm{H}$ bond are of great importance for organic synthesis, and PCET mechanism is a powerful tool to access this kind of transformations.

In 2015 , Knowles' group ${ }^{[67]}$ reported a dual catalyst system that is capable of selectively splitting the $\mathrm{N}-\mathrm{H}$ bond of $\mathrm{N}$-arylamides via PCET followed by the resulting amidyl radicals carboaminations (Scheme 15). Generally, the amide $\mathrm{N}-\mathrm{H}$ bond is stronger than allylic $\mathrm{C}-\mathrm{H}$ bond, but herein they reported that this dual catalyst system enables the formal homolysis of a stronger amide $\mathrm{N}-\mathrm{H}$ bond. In this method, Brønsted base B1 would form a hydrogen- bonded complex with amide substrate, then underwent a PCET process with the excited state of the photoredox catalyst PC1, generating an amidyl radical. This is a novel protocol for alkene carboamination via PCET mechanism. Meanwhile, this method demonstrated that concerted multisite PCET is a practical and valuable mechanism for the direct homolytic activation of relatively strong $\mathrm{N}-\mathrm{H}$ bond, and it is able to provide amidyl radical intermediates from simple anilide starting materials in a catalytic way. Most importantly, these results laid the foundation of the small-molecule catalysis platforms from the concerted PCET mechanisms, and could enable the development of some new synthetic methods.

Later, they also reported a ternary catalyst system for the unactivated olefins intramolecular hydroamidation from $N$-aryl amide derivatives (Scheme 16). ${ }^{68]}$ Similarly, this ternary catalyst system also consists of an excited state iridium complex PC1 and the same weak phosphate base
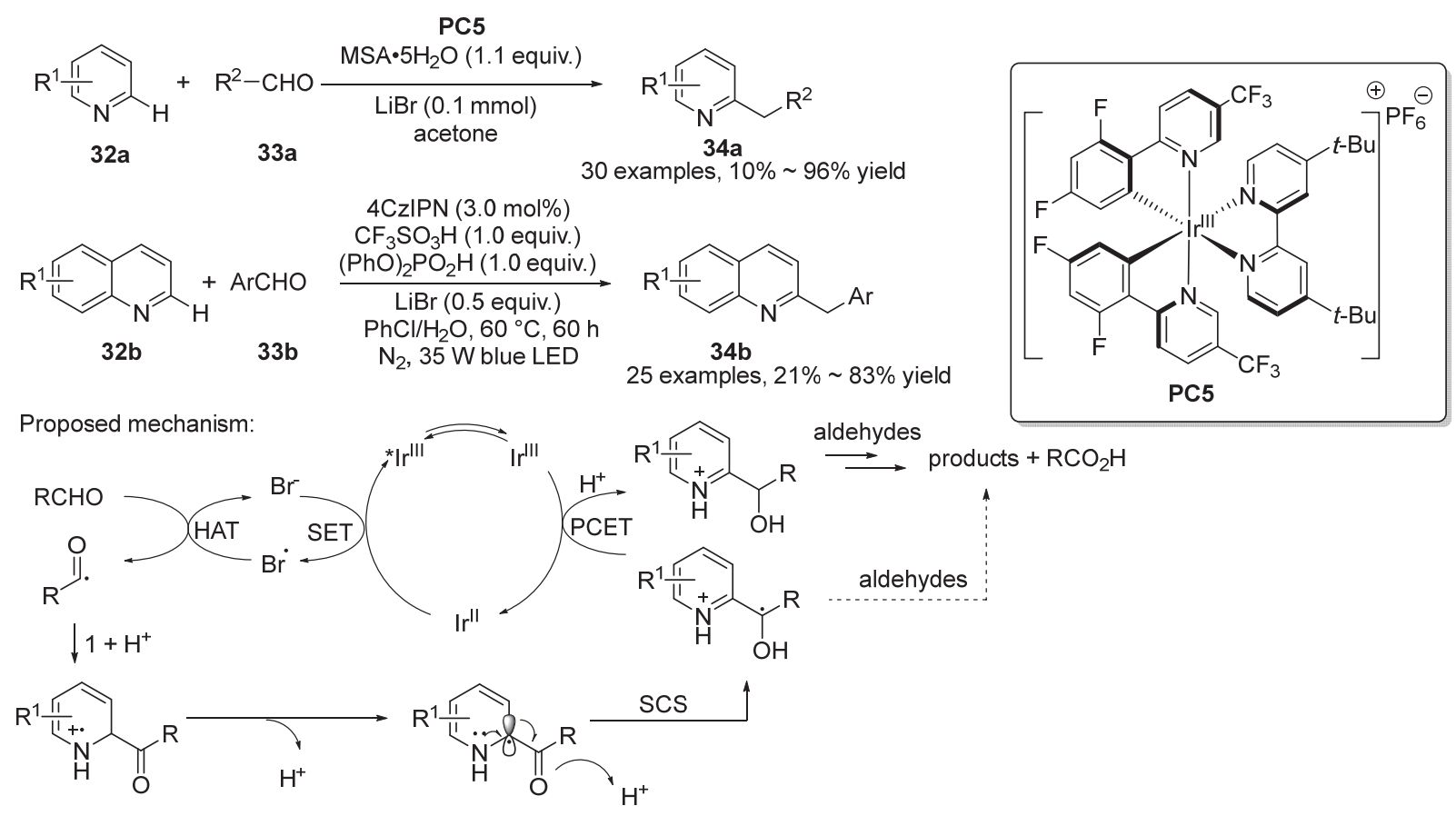

Scheme 13 Visible-light-induced reductive Minisci reaction

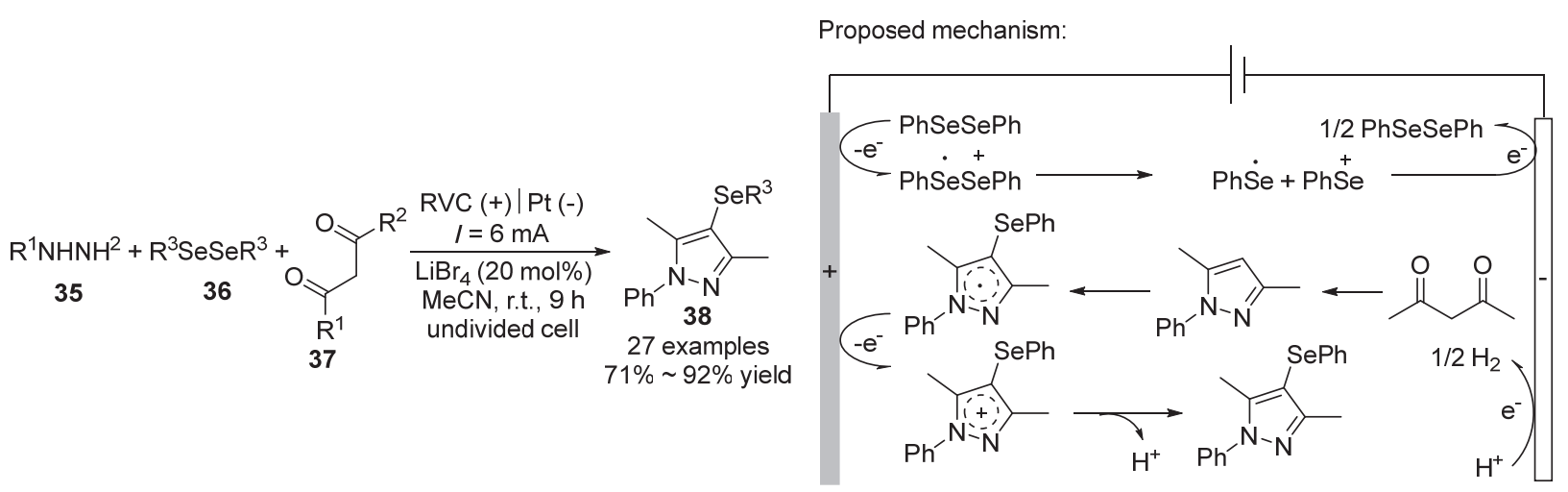

Scheme 14 Electrochemical multicomponent synthesis of 4-selanylpyrazoles 


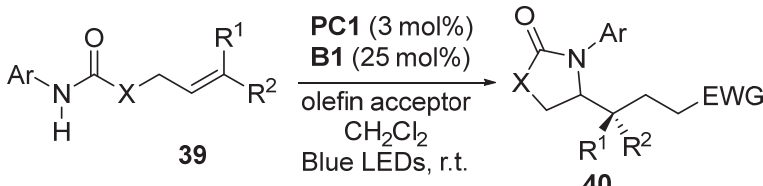

$$
\begin{aligned}
& 25 \text { examples } \\
& 50 \% \sim 95 \% \text { yield }
\end{aligned}
$$$$
\text { Proposed mechanism: }
$$

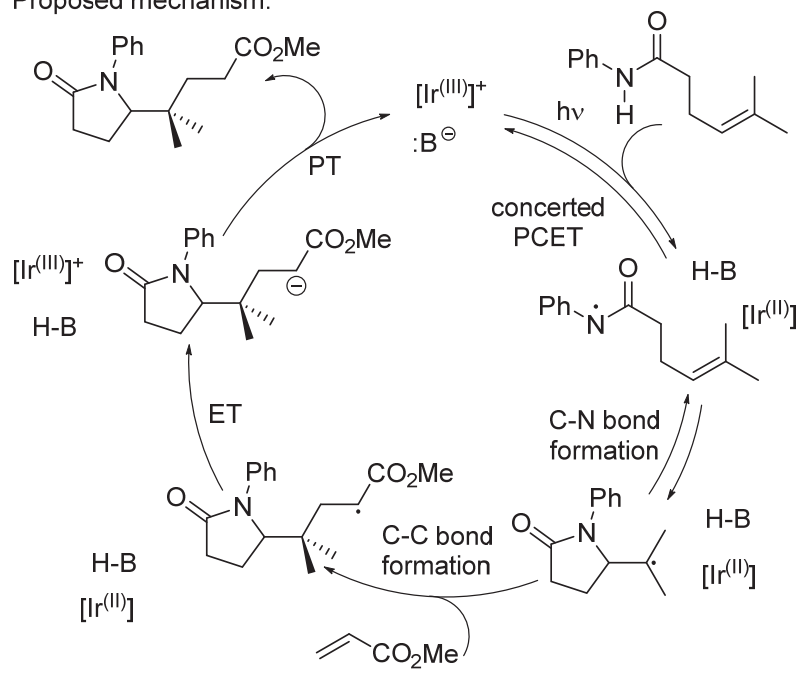

Scheme $15 \mathrm{~N}-\mathrm{H}$ activation in $\mathrm{N}$-arylamides via a dual catalyst system

B1, serving as reaction mediate for generating a reactive amidyl radical. In addition, there is an $\mathrm{H}$-atom donor catalyst for the radical reduction, and they found that thiophenol is the best among all candidates. This is a novel method for common olefin hydroamidation jointly catalyzed by three separated catalysts, and this ternary catalyst protocol enables the direct generation of amidyl from simple amide starting materials and have a large scope of olefinic reaction partners.

They soon described a catalytic $\mathrm{N}-\mathrm{H}$ bond-weakening protocol for conjugate amination through a PCET process

$$
\text { 41 }
$$

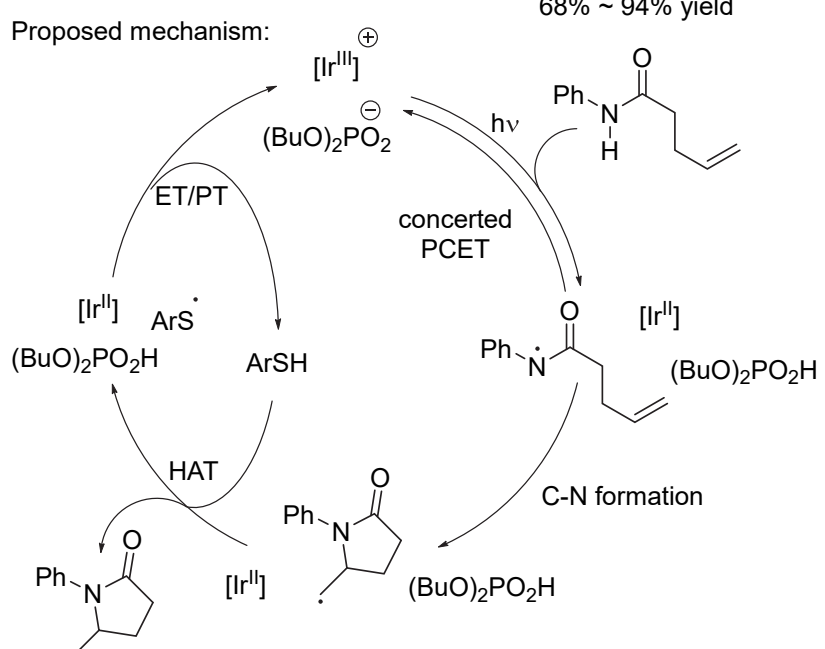

Scheme $16 \mathrm{~N}-\mathrm{H}$ activation in $\mathrm{N}$-arylamides a ternary catalyst system

(Scheme 17). ${ }^{[69]}$ In this work, the strong $\mathrm{N}-\mathrm{H}$ bond of amide was weaken by a $\mathrm{Cp}_{2} \mathrm{Ti}^{\mathrm{III}} \mathrm{Cl}$ complex which was coordinated by its carbonyl, and TEMPO• was used as an $\mathrm{H}$-atom acceptor. Through this method, the strong $\mathrm{N}-\mathrm{H}$ bond in $N$-aryl amides are destabilized by about $138 \mathrm{~kJ} / \mathrm{mol}$, which enables the homolysis of $\mathrm{N}-\mathrm{H}$ bond via PCET mechanism.

In 2016, they applied this PCET promoted $\mathrm{N}-\mathrm{H}$ bondweakening protocol to achieve the catalytic alkylation of remote $\mathrm{C}-\mathrm{H}$ bond (Scheme 18). ${ }^{[70]}$ This method exhibits a large substrates scope, even for those containing bulky groups adjacent to the amidyl nitrogen. In further research,

$$
\text { Proposed mechanism: }
$$

Scheme 17 Catalytic N-H bond-weakening protocol for conjugate amination 


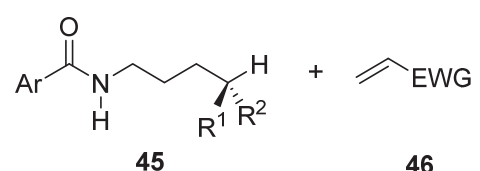

46

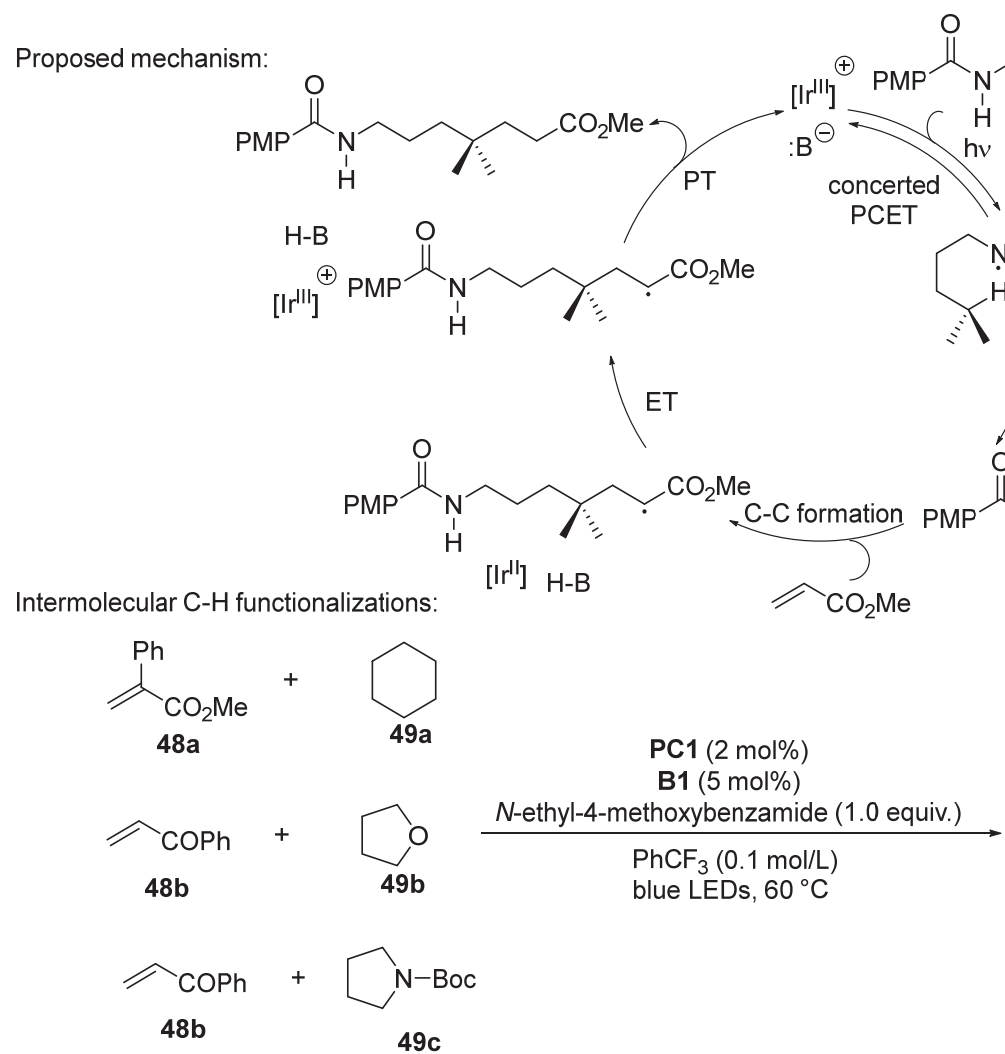

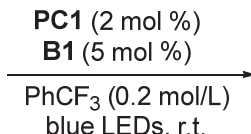

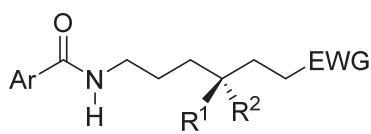

47 28 examples $29 \% \sim 82 \%$ yield 
<smiles>CCc1c[nH]c2ccccc12</smiles>

51 B2 (3 mol\%)

TEMPO• (2.0 equiv.) TIPS-EBX ( 1.5 equiv.) THF (0.05 mol/L), blue LEDs, r.t.

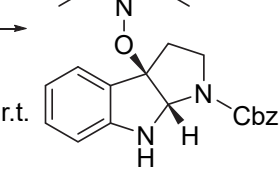

52

9 examples $59 \% \sim 81 \%$ yield

Proposed mechanism: $87 \% \sim 93 \%$ ee

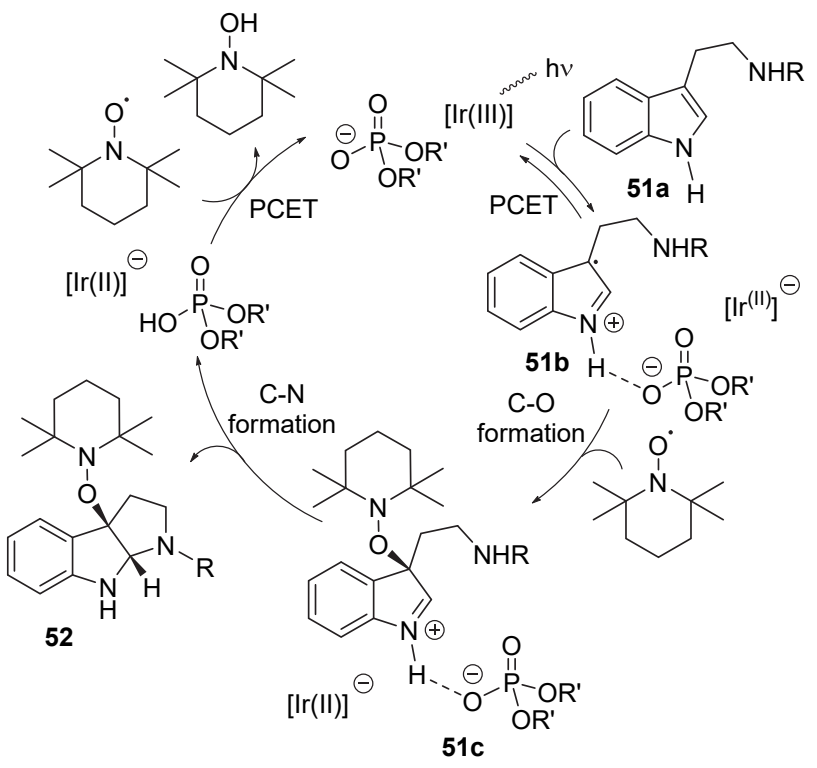

$-\mathrm{Cbz} \quad \operatorname{Ir}(\mathrm{ppy})_{3}(0.5 \mathrm{~mol} \%)$

Scheme 19 Photocatalytic method to achieve alkoxyaminesubstituted pyrroloindolines

homolysis of $\mathrm{N}-\mathrm{H}$ bond in unactivated $N$-alkyl amides via excited-state PCET mechanism, as well as shows applicability for a large range of olefin hydroamidation reactions, enabling the generation of diverse lactams and cyclic $N$-acyl amines from simple and easily available starting materials. Furthermore, this method can be modified to promote the anti-Markovnikov intermolecular hydroamidations with primary amides and the cascade polycyclizations of polyunsaturated amides

After their olefin hydroamidation of $\mathrm{N}$-alkyl amides, in 2020, Robert R. Knowles' group reported an enantioselective, radical-based method for the intramolecular hydroamination of alkenes with sulfonamides in a modified ternary catalyst system (Scheme 22). ${ }^{[74]}$ The chiral phosphate B3 was used for the control of enatioselectivity. This method accesses to the generation of enantioenriched pyrrolidine products, and it shows a high enantioselectivity for a diverse of alkene and sulfonamide substrates, even for drug-relative compounds.
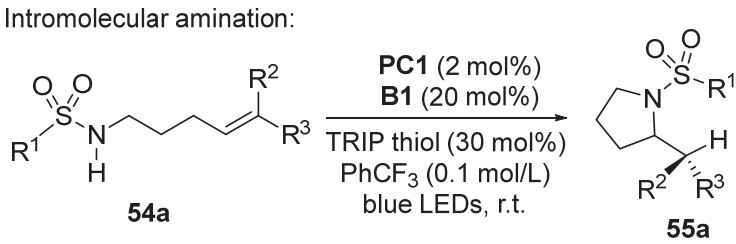

33 examples $47 \% \sim 96 \%$ yield

Intermolecular hydroamination:

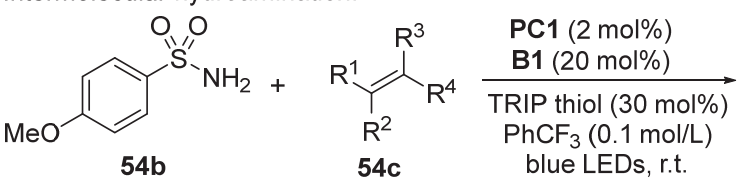

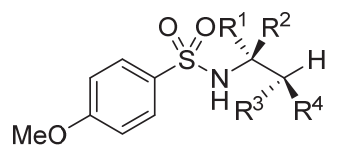

$55 b$

28 examples, $15 \% \sim 89 \%$ yield

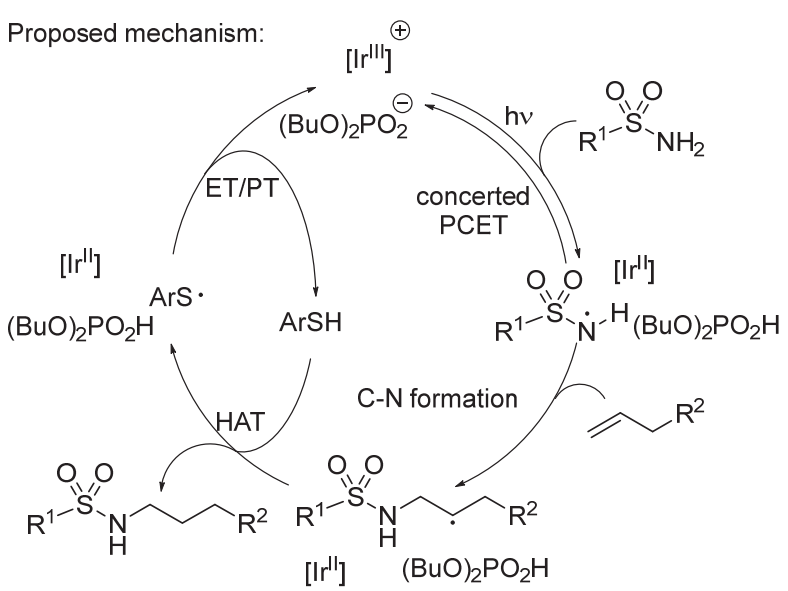

Scheme 20 Intermolecular anti-Markovnikov hydroamination of unactivated alkenes

$$
\begin{aligned}
& \text { S6 } \\
& 35 \text { examples } \\
& 63 \% \sim 97 \% \text { yield }
\end{aligned}
$$

Scheme 21 Catalytic strategy for olefin hydroamidation with $N$-alkyl amides

In 2017, Eric Meggers' group reported a novel catalytic and enantioselective conjugate addition of nitrogen-based nucleophiles to $\alpha, \beta$-unsaturated carbonyl compounds after the activation of $\mathrm{N}-\mathrm{H}$ bond via PCET mechanism (Scheme 23). ${ }^{[75]}$ A modified reaction conditions was determined, including iridium complex PC1 as photocatalyst, $\Delta-\mathbf{R h O}$ as catalyst and $\left[\mathrm{NBu}_{4}\right]^{+}\left[\mathrm{OP}(\mathrm{O})(\mathrm{OPh})_{2}\right]^{-}$(B4) as phosphate base. Notably, this method enables the catalytic and enantioselective synthesis of chiral amines without the requirement for strong base or highly nucleophilic nitrogen reagents, which are necessary in traditional methods. De- 

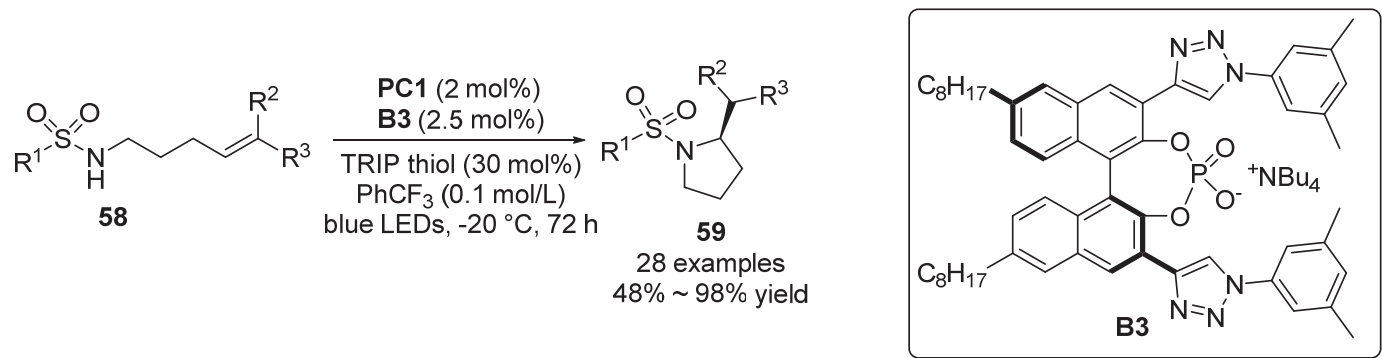

Scheme 22 Intramolecular hydroamination of alkenes with sulfonamides

spite the apparent limitations in the term of substrate scope, this method can obtain the target molecules in good yields and enantioselectivities.

In the same year, they reported a stereoselective alkylation of remote, unactivated $\mathrm{C}\left(\mathrm{sp}^{3}\right)-\mathrm{H}$ bond, which employed a combination of visible-light-induced PCET, hydrogen atom transfer, and chiral Lewis acid catalysis (Scheme 24). ${ }^{[76]}$ In this work, they chose $\boldsymbol{\Lambda}$-RhO as Lewis acid catalyst according to their previous study, with PC1 as photoredox mediator and B1 as the weak phosphate base. The reaction processes under the irradiation with two $24 \mathrm{~W}$ blue LEDs. With the optimized reaction conditions in hand, they then evaluated the substrate scope of this method and found that a variety of target molecules could be synthesized in up to $82 \%$ yield and $97 \%$ ee.

In 2018, Magnus Rueping and co-workers achieved the synthesis of alkyne- and alkene-decorated $\gamma$-lactams through a photoredox-initiated radical cascade reaction, in both good functional group tolerance and high yields (Scheme 25$).{ }^{[77]}$ The catalyst system consists of $\left[\operatorname{Ir}\left(\mathrm{dFCF}_{3}-\right.\right.$ ppy $)_{2}($ bpy $\left.)\right]^{+}\left[\mathrm{PF}_{6}\right]^{-}$(PC2) as photocatalyst and $\left[\mathrm{NBu}_{4}\right]^{+}$$\left[(\mathrm{OMe})_{2} \mathrm{PO}_{2}\right]^{-}$(B5) as Brønsted base. By introducing a sulfonyl group, the functional groups on the radical acceptor are retained successfully.

Sungwoo Hong and co-workers developed a direct strategy to the synthesis of phenanthridinone and quinolinone derivatives, which are important structures in natural products and showed a variety of optical and biological properties (Scheme 26). ${ }^{[78]}$ This method is accomplished through visible-light-promoted oxidative $\mathrm{C}-\mathrm{H}$ amidation, and employed PC2 as photocatalyst and $\left[\mathrm{NMeBu}_{3}\right]^{+}$ $\left[\mathrm{OP}(\mathrm{O})(\mathrm{OBu})_{2}\right]^{-}$(B6) as Brønsted base, and the dioxygen in air as the oxidant. This user-friendly photocatalyst sequence has a large scope of both phenanthridinone and quinolinone substrates, which can be covered to the corresponding products in good to high yield.

In 2019, Gary A. Molander's group reported the amidoarylation of unactivated olefins (Scheme 27). ${ }^{[79]}$ While amidyl radicals give new possibilities for reaction design, their formation is still lack of efficient methods, preventing

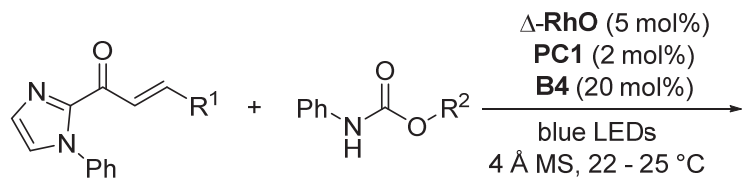

60

61

Proposed mechanism:

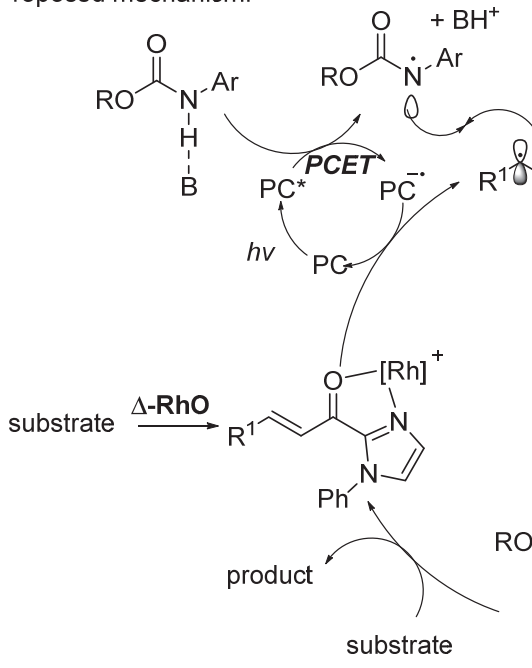<smiles></smiles>

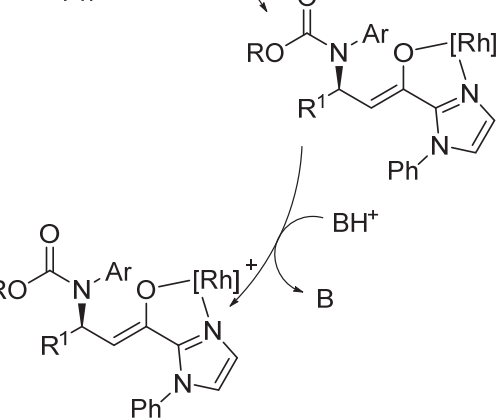<smiles>[R]OC(=O)N(CCC(=O)c1nccn1-c1ccccc1)C([R])C</smiles>

62

26 examples, $0 \sim 99 \%$ yield $94 \% \sim 99 \%$ ee

Scheme 23 Conjugate addition of nitrogen-based nucleophiles 
<smiles>[R]C=CC(=O)c1nccn1[R]</smiles>

63

28 examples, $0 \sim 82 \%$ yield

$77 \% \sim 97 \%$ ee

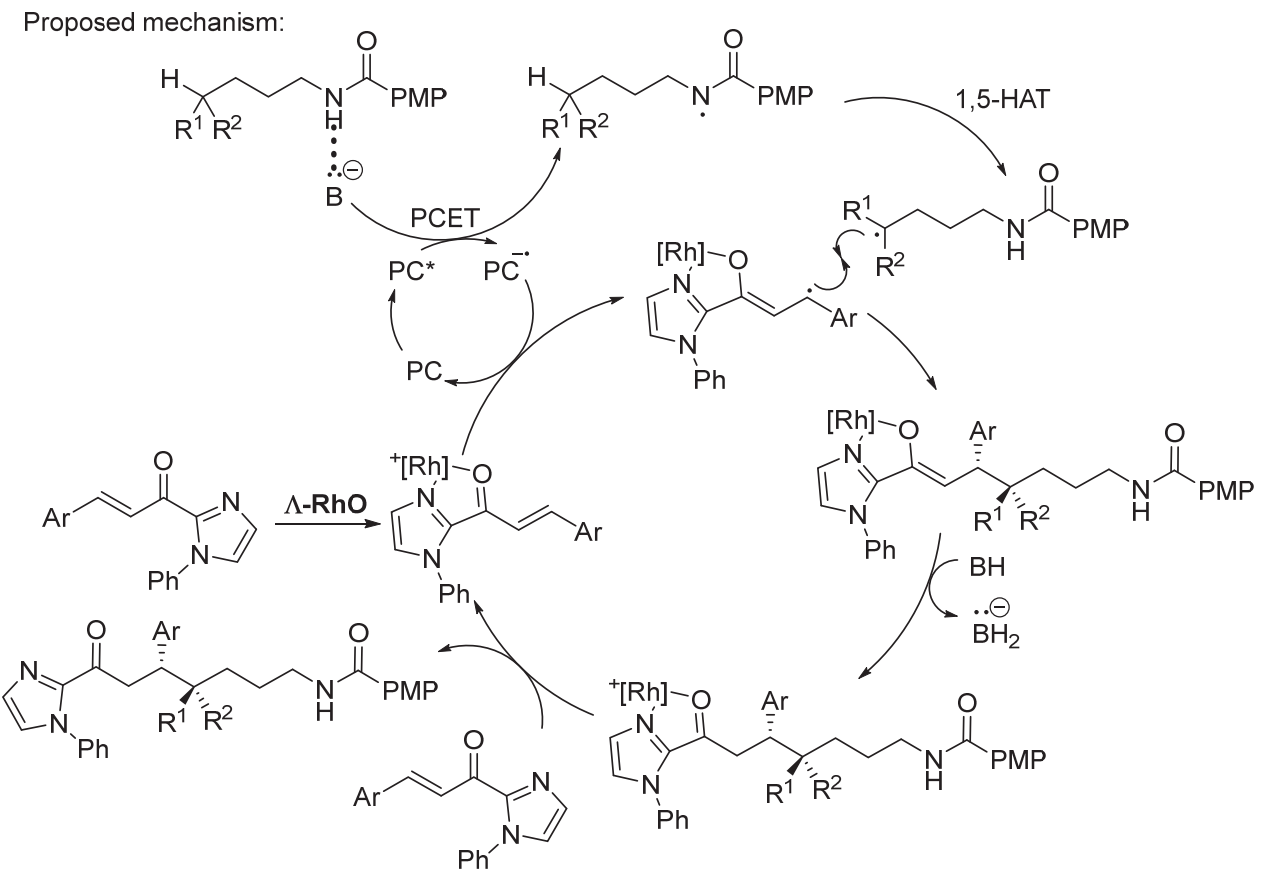

Scheme 24 Stereoselective alkylation of $\mathrm{C}\left(\mathrm{sp}^{3}\right)-\mathrm{H}$ bond<smiles>[R1]NC(=O)CC=C([R])[R]</smiles>

$\mathrm{R}^{3}=\mathrm{SO}_{2} \mathrm{Ph}$

66

Proposed mechanism:

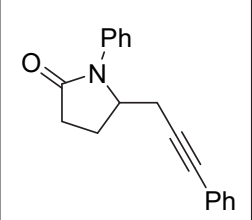

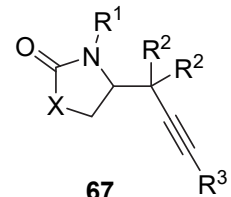

19 examples, $45 \%$ 93\% yield $\sim_{N^{-P h}}^{\mathrm{O}}$

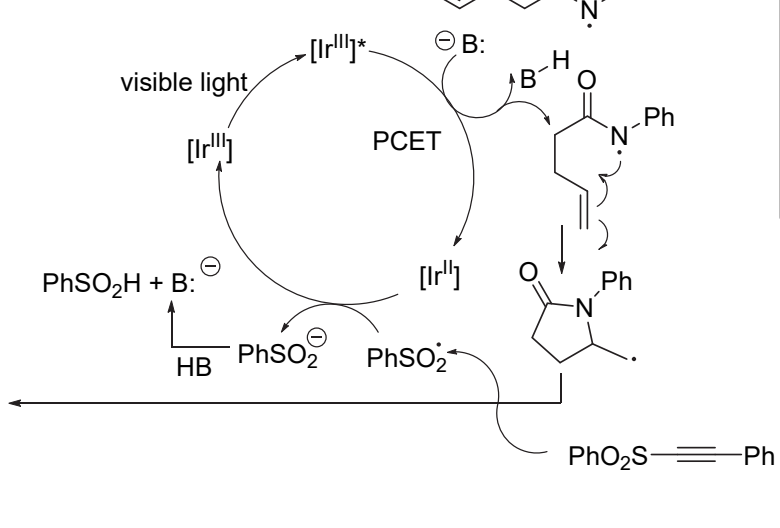

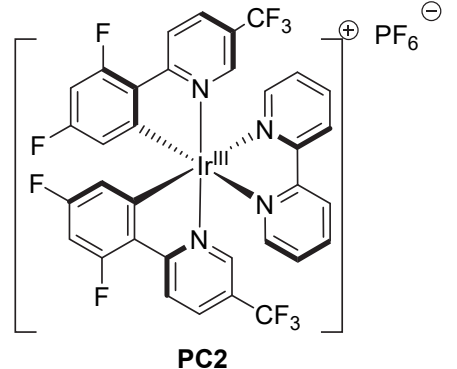

B5 $=\left[\mathrm{NBu}_{4}\right]^{+}\left[(\mathrm{OMe})_{2} \mathrm{PO}_{2}\right]^{-}$

Scheme 25 Synthesis of alkyne- and alkene-decorated $\gamma$-lactams

them from further applications in organic synthesis. To solve this problem, they used simple non-functionalized amides as substrates, with $\mathbf{P C} 2$ as photocatalyst and $\mathbf{B} 1$ as Brønsted base. In addition of stable $\mathrm{Ni}\left((\mathrm{MeO})_{2} \mathrm{bpy}\right)\left(\mathrm{H}_{2} \mathrm{O}\right)_{2-}$ 


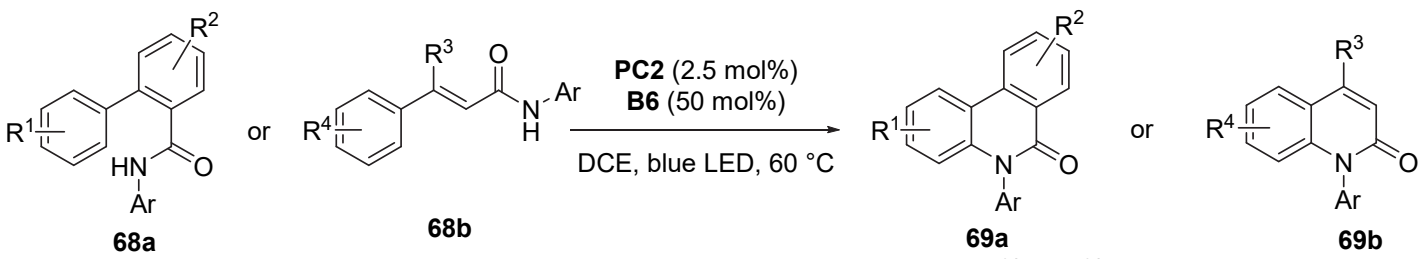

Proposed mechanism:

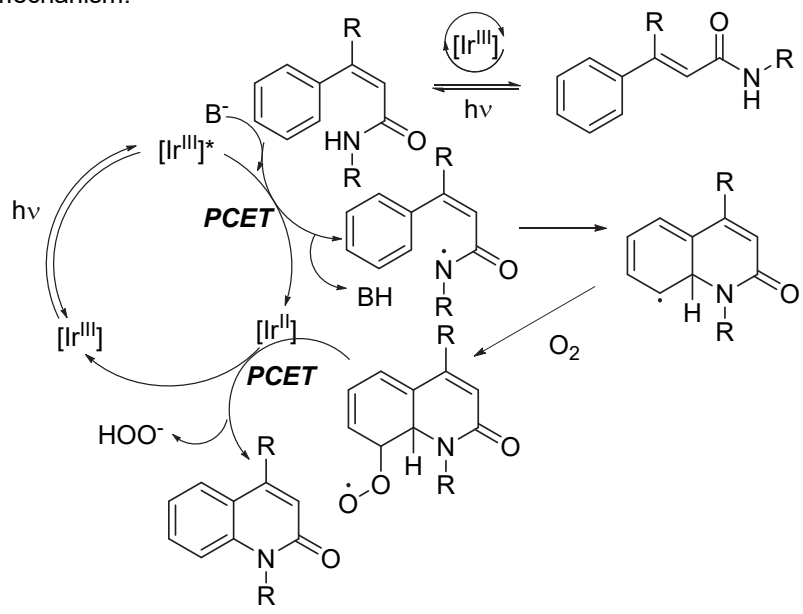

B6 $=\left[\mathrm{NMeBu}_{3}\right]^{+}\left[\mathrm{OP}(\mathrm{O})(\mathrm{OBu})_{2}\right]^{-}$

Scheme 26 Synthesis of phenanthridinone and quinolinone derivatives

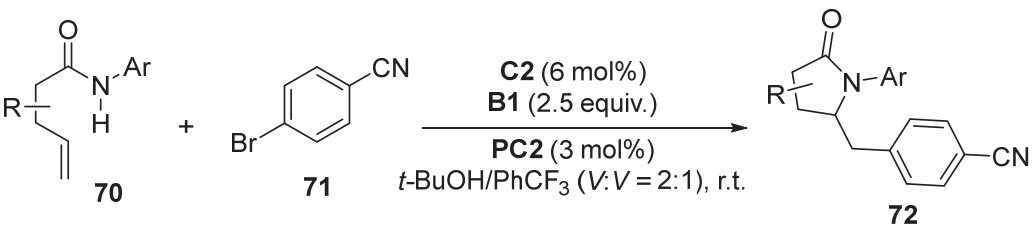

Proposed mechanism:

33 examples, $22 \% \sim 78 \%$ yield<smiles>[B]CN([CH])C(=O)CCC=C</smiles><smiles></smiles>

Scheme 27 Amidoarylation of unactivated olefin

$\mathrm{Br}_{2}(\mathbf{C 2})$ as catalyst precursor, they realized the amidoarylation of unactivated olefin, and accessed a series of molecules containing pyrrolidinone core successfully. This method shows good yields and enatioselectivities for a large scope of substrates. This is the first example for combining the concerted PCET with a nickel-catalyzed cross-coupling process together, and the application of the photoredox/ $\mathrm{Ni}$ dual-catalyst system enables the amidoarylation of unactivated olefins, leading to the construction of highly functionalized 5-membered heterocyclic structures under mild conditions.

Arylamines, especially heteroarylamines, are fundamental structures that widely exist in natural products, and are conventionally prepared by the formation of $\mathrm{C}-\mathrm{N}$ bond. However, despite their versatility, traditional methods commonly require metal catalysts, which indicates the unavoidable toxicity, and the high cost of metal reagents may prevent them from further development. In order to find out a methodology without the requirement of metal catalysts, also in $2020, \mathrm{Wu}$ and co-workers ${ }^{[80]}$ developed an access to afford heteroarylamine via radical-radical cross- coupling pathway as an alternative method for traditional transition-metal-catalyzed $\mathrm{C}-\mathrm{N}$ bond-forming reactions (Scheme 28). With a mild reaction conditions including 3DPAFIPN as photocatalyst, DABCO as base, this method leads to the photochemical, metal-free, and site-selective synthesis of a diverse range of heteroarylamine and pharmaceutical arylamines in both good to excellent yields, with 
no requirements of external oxidant or reductant.

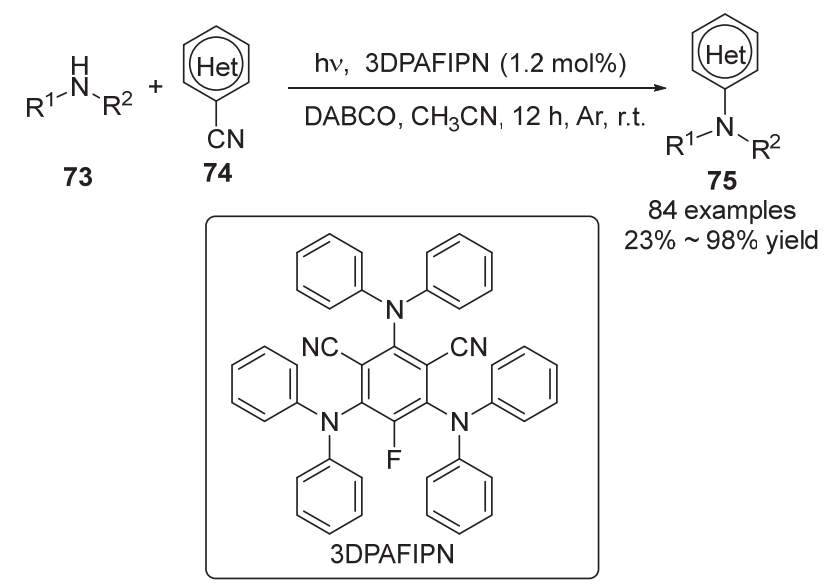

Scheme 28 Construction of heteroarylamine via radical-radical cross-coupling pathway

$\mathrm{N}-\mathrm{H}$ oxidation can also be achieved via electrochemical methods. In 2016, Hai-Chao Xu's group reported an anilide oxidation protocol promoted by an electrocatalytic way, in which dicyclopentadienyl iron was performed as the redox catalyst, and they proposed that the radical intermediate was generated via a stepwise PTET (Scheme 29). ${ }^{[81]}$ A functionalized, olefin-bearing amidyl radicals can be efficiently generated through this method, and subsequent reactions can lead to the intramolecular hydroamidation to generate lactams, cyclic carbamates and ureas.

In 2017, they also reported a metal- and reagent-free, electrochemical intramolecular oxidative amination reaction of tri- and tetra-substituted alkenes, leading to the synthesis of allylic amines, which are important synthetic building blocks for organic chemistry (Scheme 30). ${ }^{[82]}$ This newly developed method provide an efficient way for the oxidative amination of tri- and tetra-substituted hindered olefins, and is able to afford a large scope of synthetically valuable $\mathrm{N}$-heterocycles products in both high yields and diastereoselectivities. Additionally, this strategy can be easily scaled up.

And in 2018, Aiwen Lei and co-workers developed an electrochemical oxidative intramolecular $\mathrm{C}\left(\mathrm{sp}^{3}\right)-\mathrm{H}$ amination of amides (Scheme 31). ${ }^{[3]}$ Compared to conventional methods for the coupling of $\mathrm{C}\left(\mathrm{sp}^{3}\right)-\mathrm{H}$ and $\mathrm{N}-\mathrm{H}$ to access saturated nitrogen-containing compounds, in which

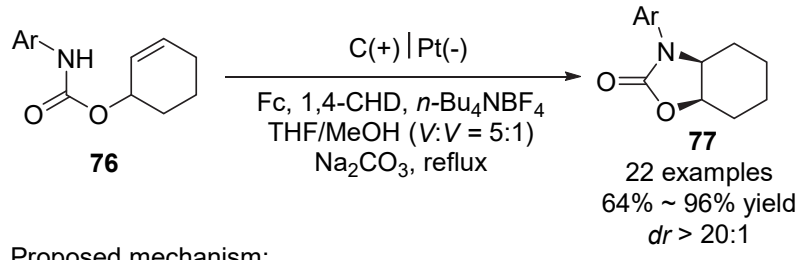

Proposed mechanism:

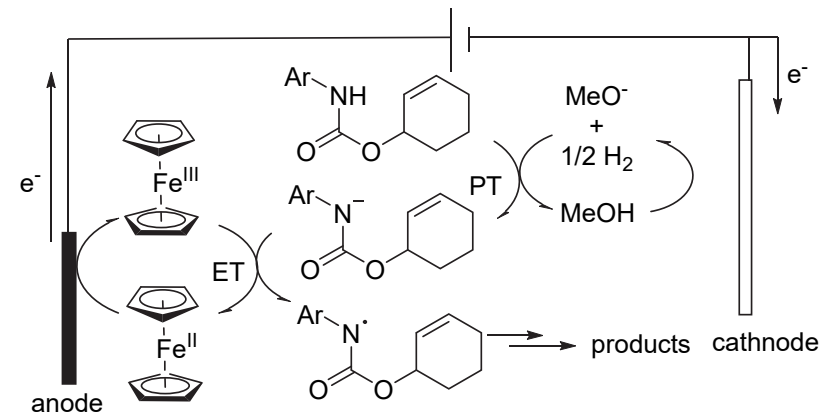

Scheme 29 Anilide oxidation promoted by electrocatalytic way

additional oxidants or halogenated reagents are often required, this method employed tetrabutylammonium acetate as not only an electrolyte, but also for promoting cleavage of the $\mathrm{N}-\mathrm{H}$ bond through the formation of intermolecular hydrogen bonds with amide substrates. A large range of $\mathrm{C}\left(\mathrm{sp}^{3}\right)-\mathrm{H}$ amination products can be afforded with good yields through this method in an oxidant- and halogenation-free way.

In 2020, Stahl's group ${ }^{[84]}$ reported an electrochemical strategy for the oxidative homo-coupling of benzophenone imine via PCET process (Scheme 32). This method leads to the synthesis of hydrazine, which is important for industry. With $\left[\mathrm{MeBu}_{3} \mathrm{~N}\right]^{+}\left[\mathrm{OP}(\mathrm{O})(\mathrm{OBu})_{2}\right]^{-}$as the phosphate base, $n-\mathrm{Bu}_{4} \mathrm{NPF}_{6}$ as supporting electrolyte, the desired product can be accessed from 4,4'-difluorobenzophenone imine in $84 \%$ yield. Besides, Stahl's group also demonstrated two other complementary methods to achieve $\mathrm{N}-\mathrm{N}$ coupling of benzophenone imine through an electrochemical way, which involves PCET on the surface of electrodes. Notably, imines are used as the alternative of ammonia substrates to enable the reaction stop at the formation of a single $\mathrm{N}-\mathrm{N}$ bond, otherwise a kinetically facile and thermodynamically favorable oxidation of hydrazine to dinitrogen would occur.

Notably, a metal- and catalyst-free electrochemical method for oxidation reactions was reported in 2020. Zhixiong Ruan and co-workers developed a direct electro-chemical

$$
\begin{aligned}
& \text { undivided cell, } 10 \mathrm{~mA} \\
& \downarrow \text { PCET } \\
& 37 \text { examples, } 48 \% \sim 97 \% \text { yield } \\
& \longrightarrow
\end{aligned}
$$

Scheme 30 Electrochemical intramolecular oxidative amination reaction of tri- and tetra-substituted alkenes 


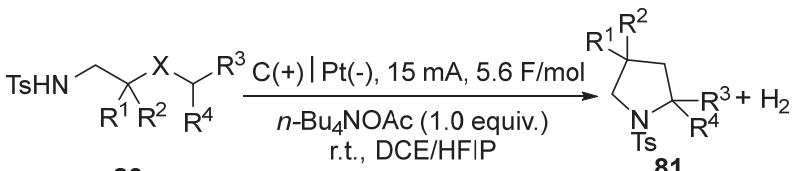

$$
\begin{aligned}
& 80 \\
& 35 \text { examples } \\
& 35 \% \sim 98 \% \text { yield }
\end{aligned}
$$

Scheme 31 Electrochemical oxidative intramolecular $\mathrm{C}\left(\mathrm{sp}^{3}\right)$ $\mathrm{H}$ amination of amides<smiles>N=C(c1ccc(F)cc1)c1ccc(F)cc1</smiles>

82

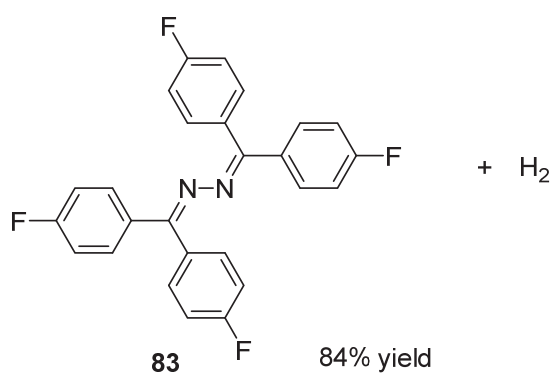

Proposed mechanism:<smiles>N=C(Br)Br</smiles>

dimerization product

Scheme 32 Oxidative homo-coupling of benzophenone imine

synthesis of annulated medium-sized lactams via cleavage of $\mathrm{C}-\mathrm{C}$ bond in catalyst-free conditions (Scheme 33). ${ }^{[85]}$ This approach enables ring expansion through an unique remote amidyl migration under mild conditions, and shows a diverse scope, high yield, and broad functional group tolerance. The electron and proton transfer on the surface of the electrodes should be under different PCET processes.

\section{$3.3 \mathrm{O}-\mathrm{H}$ bond}

$\mathrm{O}-\mathrm{H}$ bond have a relative strong bond energy among $\mathrm{X}-\mathrm{H}$ bond, and their homolysis followed by functionalization under mild conditions have been a long-standing goal in organic chemistry. Recently, with the PCET processes, several methods have been developed. For example, O-centered radicals generated by the activations of strong $\mathrm{O}-\mathrm{H}$ bond in alcohol via PCET mechanism could be an important mediator for the subsequent $\beta$-scission to the adjacent $\mathrm{C}-\mathrm{C}$ bond, leading to the formation of a new carbonyl group. Thus, PCET-based $\mathrm{O}-\mathrm{H}$ activations are of great significance for organic synthesis and have drawn more and more attention from organic chemists.

Oxidation reactions have been an attractive topic for a long period of time, among which the oxidation of alcohols
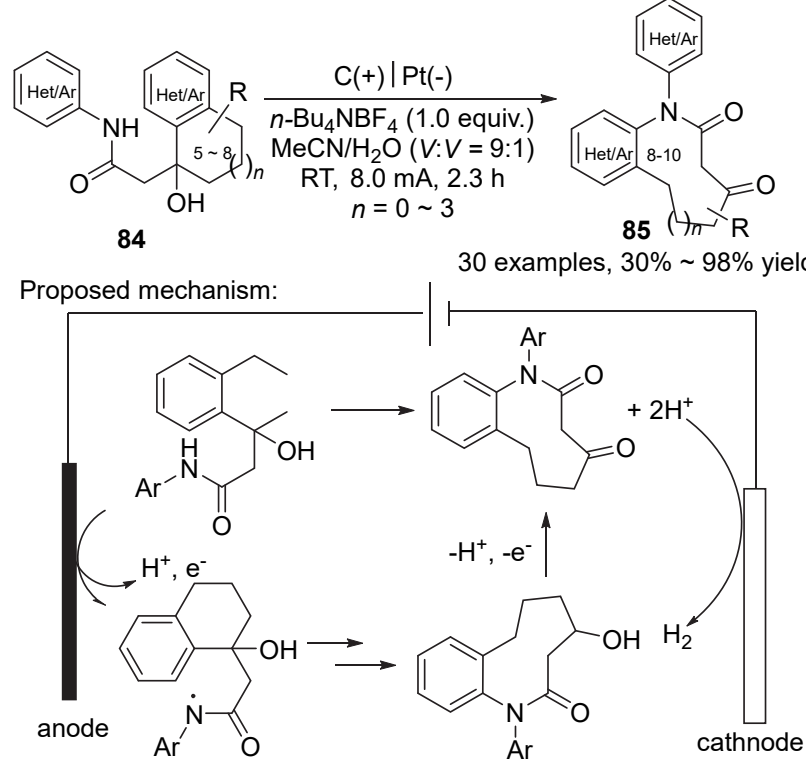

Scheme 33 Metal- and catalyst-free electrochemical synthesis of annulated medium-sized lactams

to aldehydes, ketones, and carboxylic acids are one of the most important issues. ${ }^{[86-88]}$ However, limited by their synthetic scope and practical factors, aerobic oxidation reactions application in organic chemistry was constrained. In order to solve this problem, Stahl's group have developed a new catalyst system mainly consist of copper and TEMPO•. This kind of catalyst system is mainly applied into the aerobic oxidation of alcohols.

Their earliest example on the copper/TEMPO• catalyzed aerobic oxidation reactions of alcohols was reported in 2011 (Scheme 34). ${ }^{[89]}$ In this work, Stahl and co-workers successfully transferred a large range of primary alcohols, such as allylic, benzylic, and aliphatic derivatives, to their corresponding aldehydes at room temperature with ambient air as the oxidant. This method leads to a highly primary alcohol-selective oxidation under mild conditions, without the requirement of pure oxygen, and is compatible with a wide range of functional groups.

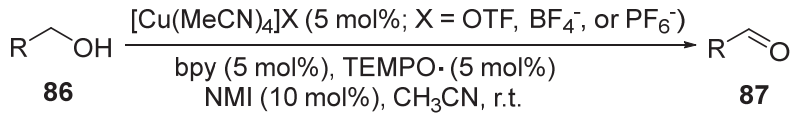

Scheme 34 Copper/TMEPO catalyzed aerobic oxidation reactions of alcohols

Based on the work described above, in 2013, Stahl's group launched a further study and reported a scalable, continuous-flow aerobic oxidation of primary alcohols, also under the same copper/TEMPO catalyst system (Scheme 35). ${ }^{[90]}$ Dilute oxygen source $\left(9 \% \mathrm{O}_{2}\right.$ in $\left.\mathrm{N}_{2}\right)$ was used as oxidant in this work. This method allows a larger scale application with lower catalyst loading.

Later, they also developed an efficient chemoselective metal-free aerobic oxidation for secondary benzylic alcohol 


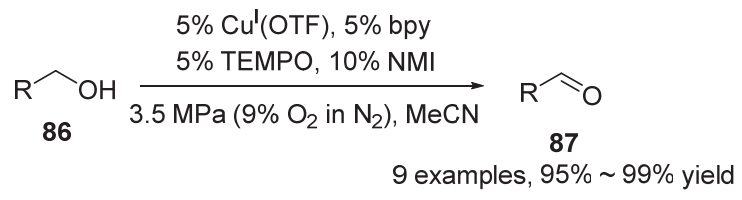

Scheme 35 Scalable, continuous-flow aerobic oxidation of primary alcohols

in lignin, which is a biopolymer and represents a potential for the production of organic chemicals as it is one of the few natural sources of high-volume aromatics (Scheme $36) .{ }^{[91]}$ In this work, they adapt their conventional catalyst system to a new one consist of 4-acetamido-TEMPO- in combination with $\mathrm{HNO}_{3}$ and $\mathrm{HCl}$. This method provides a highly effective way for the chemoselective aerobic oxidation of benzylic $2^{\circ}$ alcohols. Moreover, by combining this catalyst strategy to a subsequent oxidation step, a $\mathrm{C}-\mathrm{C}$ bond cleavage proceed could be achieved in the model compound of the biomass like lignin.

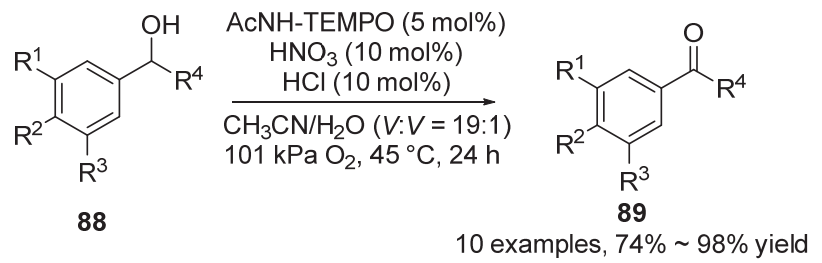

Subsequent oxidation:

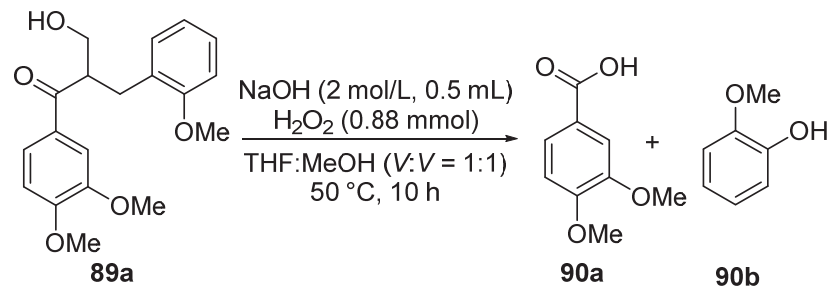

Scheme 36 Chemoselective metal-free aerobic oxidation for secondary benzylic alcohol in lignin

Besides TEMPO•, Stahl's group employed ABNO as analogous catalyst as well. In 2015, they reported an improved copper/nitroxyl catalyst system for the oxidation of functionalized primary and secondary alcohols to corresponding aldehydes and ketones (Scheme 37). ${ }^{\left[{ }^{[2]}\right.}$ This improved catalyst system consists of copper/ABNO/NMI, among which the ABNO works as an organic nitroxyl redox catalyst. Compared to their previously developed copper/TEMPO• catalyst system which is particularly effective for primary alcohols, this copper/ABNO system displays chemoselectivity for both primary and secondary alcohols. This method also exhibits a broad substrate scope and good functional group compatibility, and undergoes a similar mechanism to that of TEMPO•. To be specific, $\mathrm{CuI}$ is used to be the copper catalyst in this protocol, which is less expensive than traditional copper(I) salts.

In 2018, Stahl's group applied this TEMPO• catalyst into the electrochemical oxidation of alcohols and aldehydes to the generation of carboxylic acids (Scheme 38). ${ }^{[93]}$ Com-

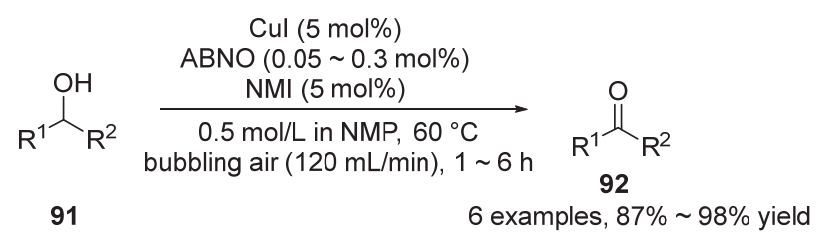

Scheme 37 Improved copper/nitroxyl catalyst system for the oxidation of functionalized primary and secondary alcohols

pared to their traditional catalyst system which include TEMPO• and copper complex, this newly developed method requires no metal reagents. Moreover, this reaction proceeds under mild conditions, which enables the retention of stereochemistry adjacent to the site of oxidation. This strategy provides an effective and scalable route for the preparation of carboxylic acids via a simple and environmentally friendly way.
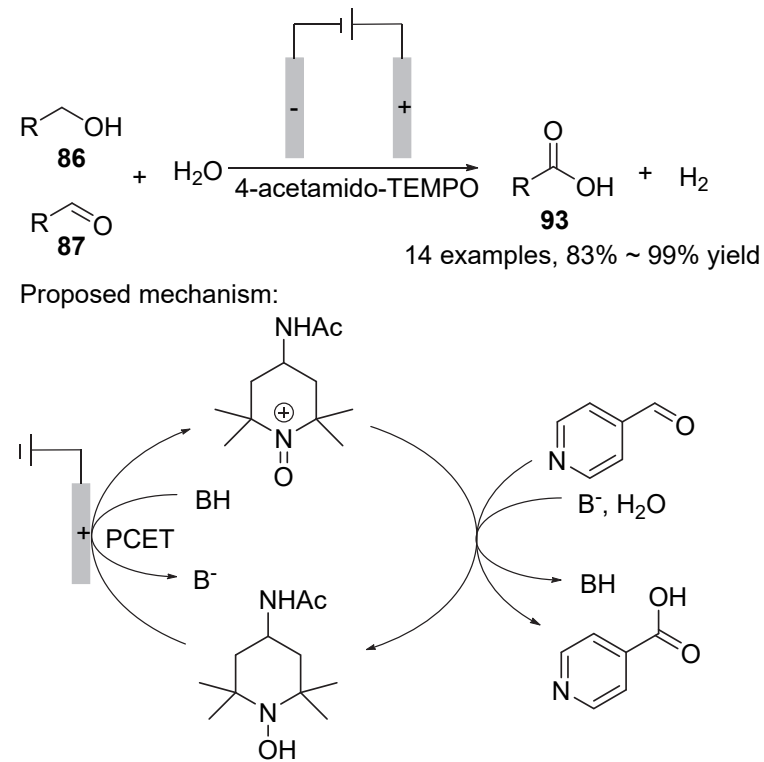

Scheme 38 Electrochemical oxidation of alcohols and aldehydes catalyzed by TEMPO•

In 2019, Stahl's group further achieved the oxidation of primary alcohols in lignin via an electrochemical aminoxyl-mediated and metal-free way (Scheme 39). ${ }^{[94]}$ This protocol employs TEMPO• and 4-acetamido-TEMPO• as catalytic mediators, and exhibits a high chemoselectivity for oxidation of primary alcohols groups. Notably, the modified lignin generated from this method is adapted to be susceptible to depolymerization, allowing its conversion to the monomeric aromatic compounds in good yields.

Photocatalytic systems can also oxidize $\mathrm{O}-\mathrm{H}$ bond via PCET processes. At the same year, Knowles' group reported a general ring-cleavage and oxidative method for the light-driven isomerization of cyclic aliphatic alcohols to linear carbonyl compounds (Scheme 40). ${ }^{[5]}$ This method promotes isomerizations to proceed against an energetic gradient via a photochemical approach. 


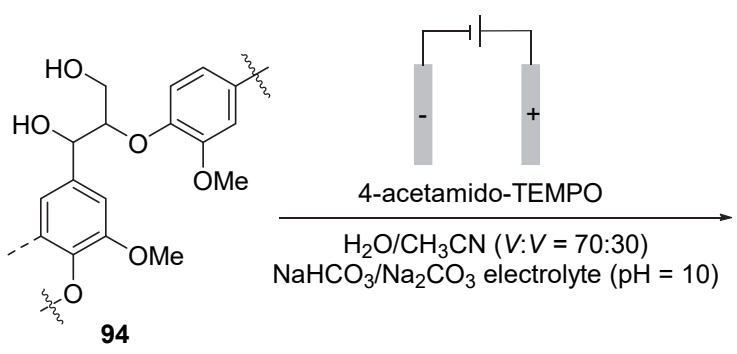<smiles>COc1cc(C(C)(C)C)ccc1OC(C(=O)O)C(O)c1cc(C)c(OC(C)(C)C)c(OC)c1</smiles>

95

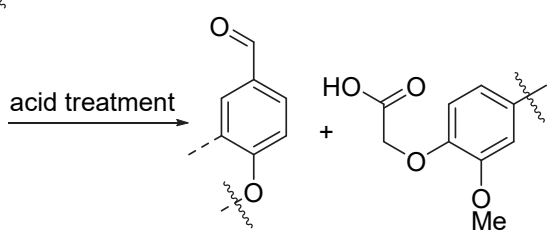

96b
Scheme 39 Oxidation of primary alcohols in lignin via electrochemical aminoxyl-mediated pathway

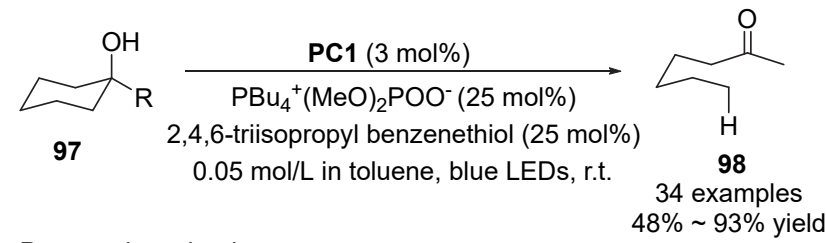

Proposed mechanism:

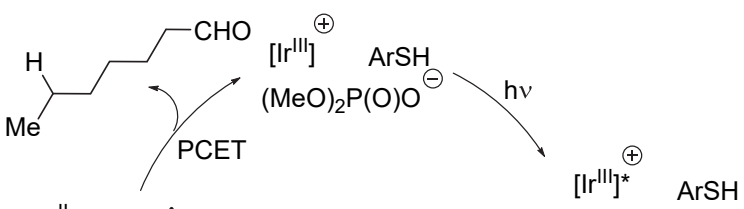

$\left[\mathrm{Ir}^{\| \prime}\right] \quad \mathrm{ArS}^{\circ}$

$(\mathrm{MeO})_{2} \mathrm{P}(\mathrm{O}) \mathrm{OH}$
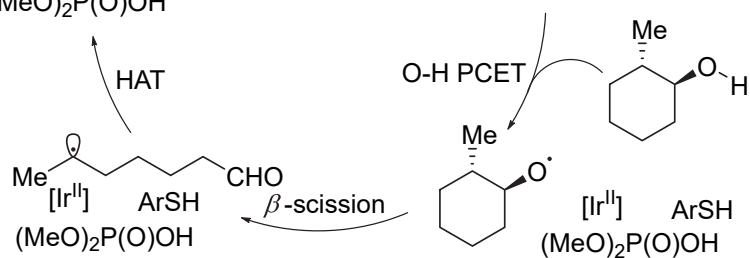

Scheme 40 Ring-cleavage and oxidative method for the isomerization of cyclic aliphatic alcohols

They later reported a catalytic method for the modular ring expansion of cyclic aliphatic alcohols, in which activations of alcohol $\mathrm{O}-\mathrm{H}$ bond via PCET mechanism are involved (Scheme 41). ${ }^{[96]}$ The substituted ethenyl group located on the $\alpha$-position of the hydroxyl enables a $\mathrm{C}-\mathrm{C}$ $\beta$-scission after the generation of alkoxy radical, furnishing a carbonyl group and alkyl radical, and the alkyl radical can therefore attack the ethenyl group to form a new $\mathrm{C}-\mathrm{C}$ bond, resulting into the ring expansion product. With different substituent groups on the ethenyl, the reacting position of alkyl radical would be different and lead to the $n+1$ or $n+2$ expansion.

With this well-established strategy in which $O$-centered radical is generated via PCET activation of $\mathrm{O}-\mathrm{H}$ bond in
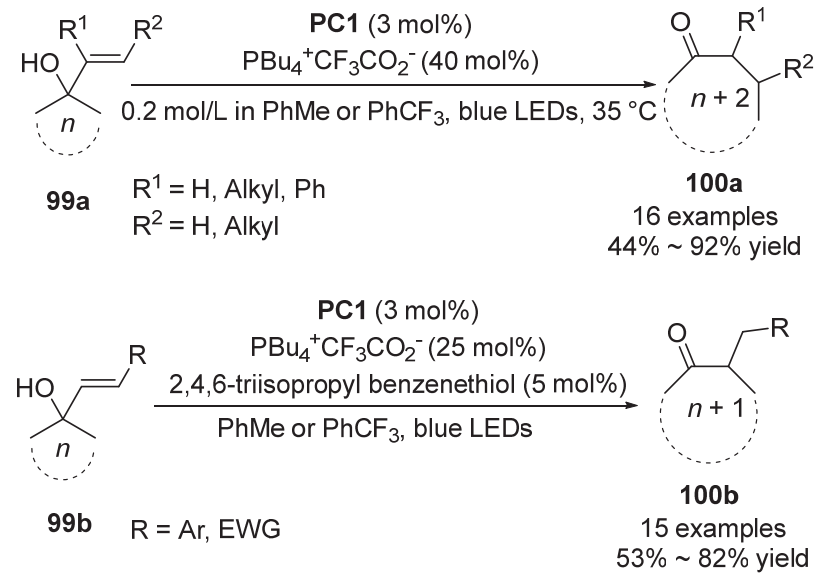

Scheme 41 Modular ring expansion of cyclic aliphatic alcohols

alcohol, and followed by a $\mathrm{C}-\mathrm{C} \beta$-scission, soon they reported a catalytic, light-driven method for the redox-neutral depolymerization of native lignin at room temperature (Scheme 42). ${ }^{[97]}$ This method is promoted only by visible light and with no requirements for stoichiometric chemical reagents, and produces no waste. This one-step protocol proceeds under mild reaction conditions, leading to the synthesis of a well-defined and isolable set of monomeric arene products.

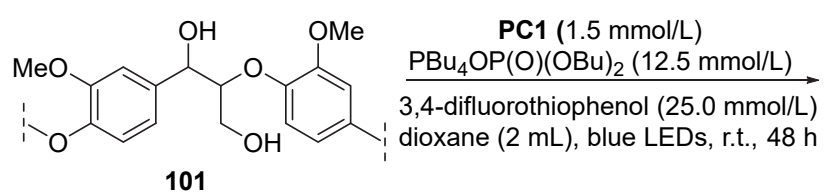<smiles>COc1cc(C=O)ccc1OCCO</smiles>

Scheme 42 Catalytic and light-driven method for the redoxneutral depolymerization of native lignin

To use this strategy of PCET introduced fragment, the transition metal catalyzed cross-coupling to from $\mathrm{C}-\mathrm{C}$ bond can be achieved. In 2020, Rueping and co-workers ${ }^{[98]}$ reported a general visible-light-mediated strategy for the activation of cyclic alcohols in order to access the remote arylation of ketones via photoredox-mediated multisite PCET (MS-PCET) combined with nickel catalysis (Scheme 43). In the reaction path way, the cyclic alcohols are broken via a PCET reaction and subsequently form carbonyl intermediates, followed by the reactions with aryl halides to lead to the remote site-specific arylation of ketones and access the final products. This $\mathrm{C}-\mathrm{C}$ bond activation cross coupling strategy has a mild condition, and can be utilized in a diverse set of cyclic alcohols substrates.

Besides the catalytic system above, the oxidative PCET processes of $\mathrm{O}-\mathrm{H}$ bond can also make annulation reactions. The Knowles' group applied their ternary catalyst 

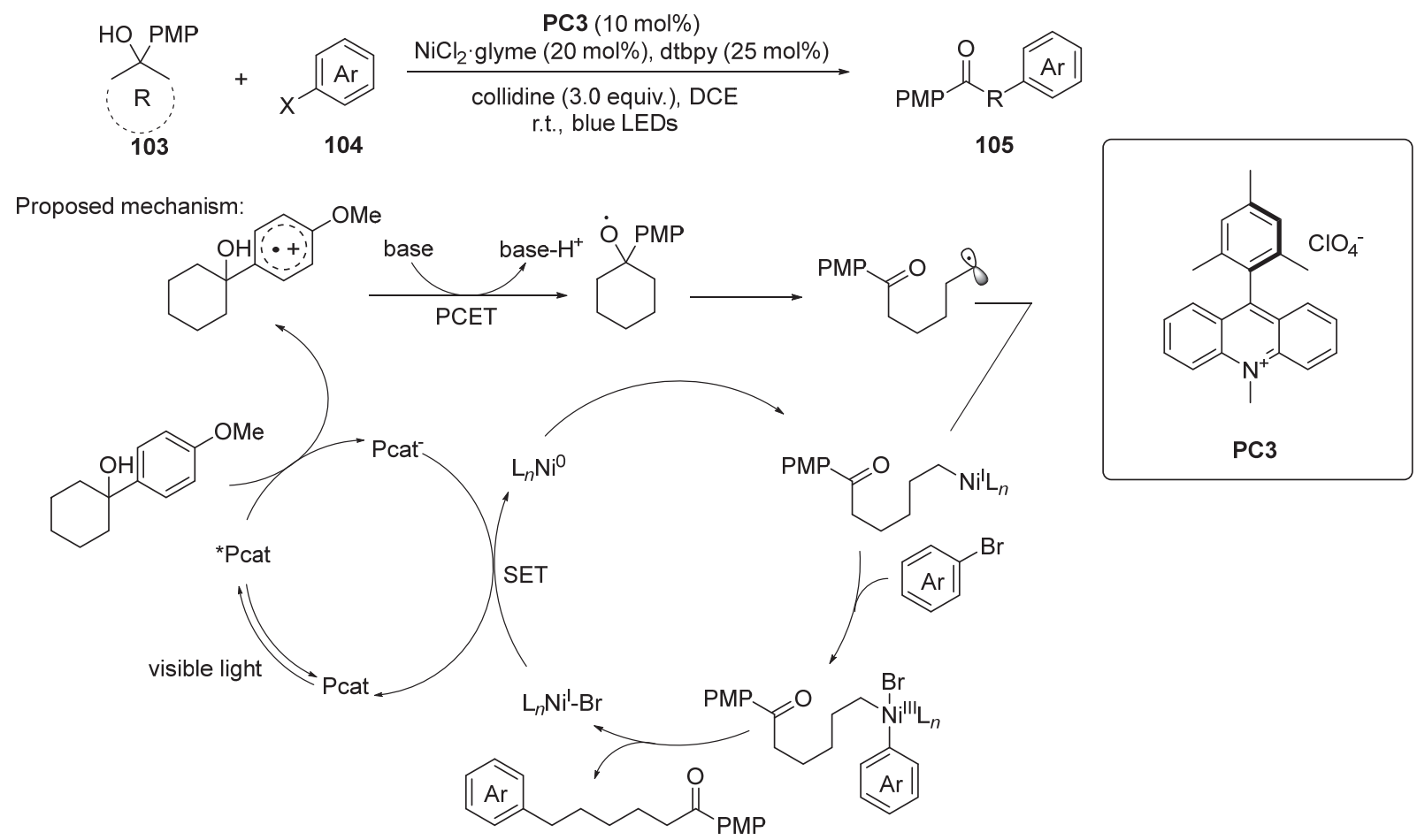

Scheme 43 Remote arylation of ketones via photoredox-mediated multisite PCET

system involving an $\mathrm{Ir}^{\mathrm{III}}$-based photoredox catalyst, a Brønsted base catalyst, and a hydrogen-atom transfer (HAT) co-catalyst, into the activation of $\mathrm{O}-\mathrm{H}$ bond via PCET mechanism. In 2020, they reported a catalytic, light-driven method for the intramolecular hydroetherification of unactivated alkenols (Scheme 44) ${ }^{[99]}$ This method can lead to the construction of oxygen-containing heterocycles which are common structures in a various of natural products.
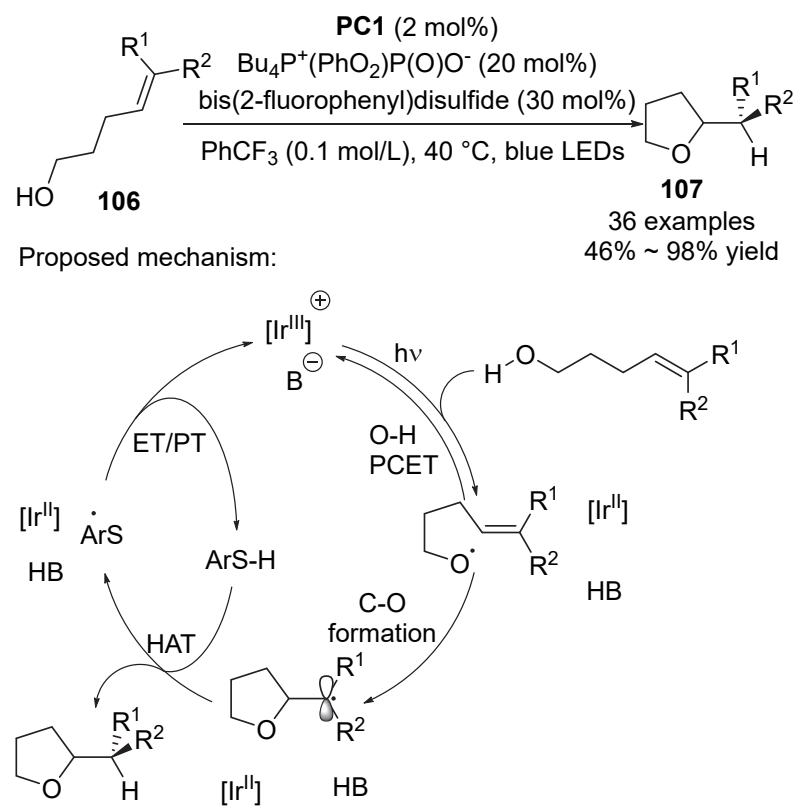

Scheme 44 Catalytic and light-driven method for the intramolecular hydroetherification of unactivated alkenols

\section{$3.4 \mathrm{P}-\mathrm{H}$ bond}

Recently, the bond-dissociation free-energy (BDFE) of $\mathrm{P}-\mathrm{H}$ bond was examined by Cheng's group ${ }^{[46]}$ via a stepwise proton transfer and electron transfer, which could evaluate their hydrogen atom donabilities. One example of the PCET-promoted $\mathrm{P}-\mathrm{H}$ bond activation is the construction of $Z$-alkenes, which are fundamental structures in many biologically active natural products. ${ }^{[100-103]}$ Addition to alkynes is a straightforward, atom-economical, and environmentally friendly method to access the synthesis. ${ }^{[104-108]}$ However, the formation of $Z$-alkenes via addition to alkynes is still vacant but highly desirable. In 2018, Aiwen Lei and co-workers reported the direct synthesis of $Z$-alkenylphosphine oxides via visible-light-induced radical addition to alkynes in water (Scheme 45). ${ }^{[109]}$ In their strategy, with the photoexcited catalysts, a phosphinoyl radical (I) is formed via PCET oxidation. This method requires no transition metal reagents, and can synthesize the desired $Z$-alkenylphosphine oxide products via radical addition to alkynes at room temperature in a high stereoselectivity. Moreover, the reaction features a broad functional-groups tolerance and gram-scale synthesis. This work offers a highly efficient way to the construction of $Z$-alkenylphosphine oxides.

In 2021, Bing Yu and co-workers developed a visible-light-induced PCET strategy for the generation of phosphorus-centered radicals (Scheme 46). ${ }^{[110]}$ 2,4,5,6Tetrakis(3,6-di-tert-butyl-9H-carbazol-9-yl)isophthalonitrile (4CzIPN-tBu) (PC4) was used as photocatalyst in this strategy. With the catalysis of 4CzIPN-tBu, which was be used as an efficient photocatalyst for the phosphorusradical- 


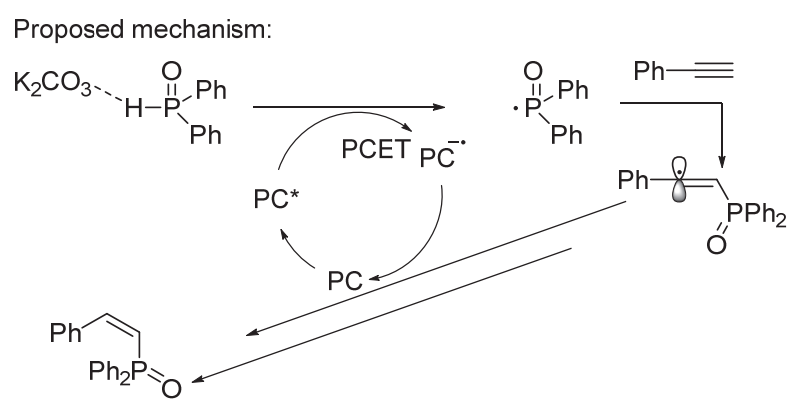

Scheme 45 Synthesis of $Z$-alkenylphosphine oxides via visible-light-induced radical addition
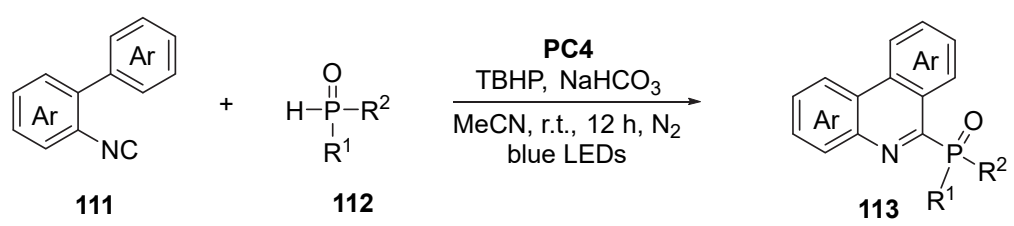

24 examples, trace $\sim 80 \%$ yield

Proposed mechanism:

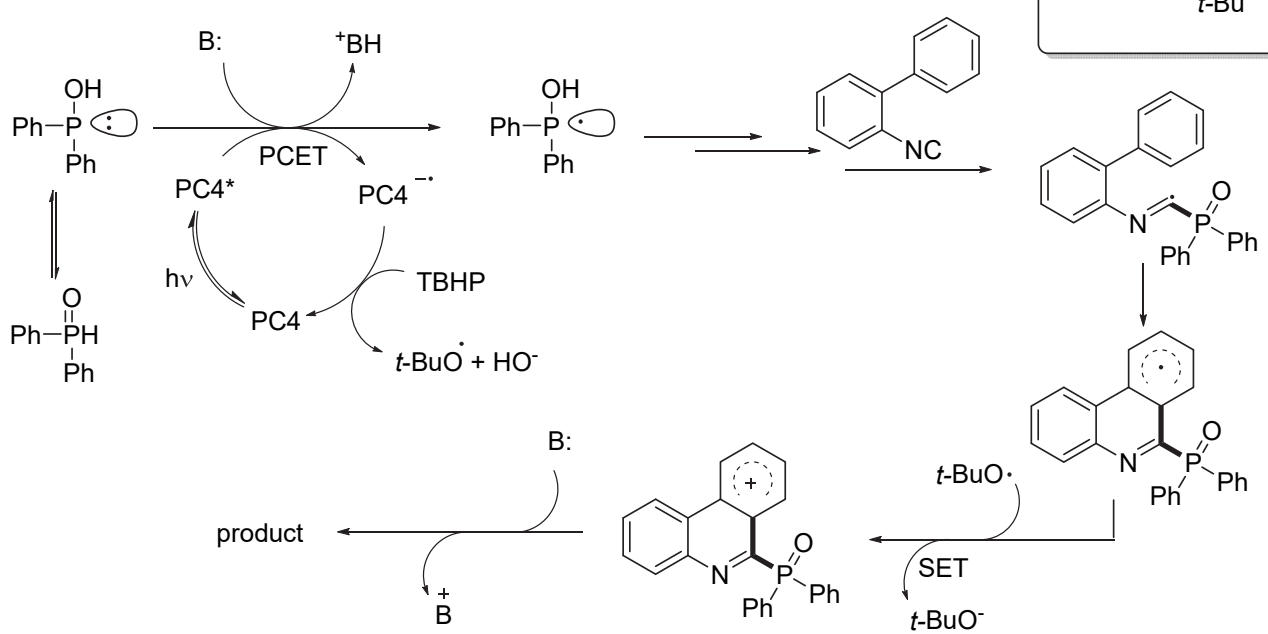

Scheme 46 Visible-light-induced PCET strategy for the generation of phosphorus-centered radicals

initiated cascade cyclization reaction of isocyanides, a diverse range of phosphorylated aromatics such as quinolines, phenanthridines, as well as benzothiazoles could be accessed successfully. This could also be a valuable guide for the development of transition-metal-free photocatalyst strategies in order to the efficient organic synthesis in the future.

\section{$3.5 \mathrm{~S}-\mathrm{H}$ bond}

A series of studies on the generation of thiyl radicals promoted by the activation of $\mathrm{S}-\mathrm{H}$ bond in thiol substrates via PCET mechanism have been reported. Thiols are conventionally used as HAT agents in free radical chemistry, while recent research shows they can also be applied in MS-PCET chemistry. In 2013, Oliver S. Wenger and co-workers investigated the PCET mechanism with thiophenols (Scheme 47).$^{[111]}$ To get a further exploration of this process, Martin Kuss-Petermann and co-workers launched a suite of PCET reactions using a series of thiophenols, and with a photoexcited $\mathrm{Ru}(\mathrm{II})$ complex that acts as a cooperated electron or proton acceptor. Depending on the exact nature of the thiophenol, PCET occurs through three different reaction mechanisms, namely, ET-PT (stepwise electron transfer-proton transfer, for $\mathrm{Me}_{3} \mathrm{PhSH}$ ), PT-ET (stepwise proton transfer-electron transfer, for $\mathrm{F}_{5} \mathrm{PhSH}$ ), and CPET or CEPT (concerted proton-electron transfer, for $\mathrm{Cl}_{3} \mathrm{PhSH}$, although not completely clear). This work demonstrates that the mechanistic possibilities may lead to phenylthiyl radicals.

The thiol radical intermediates generated from the homolysis of $\mathrm{S}-\mathrm{H}$ bond via PCET mechanism have been applied to achieve the activations of some other bonds for a long time. ${ }^{[112-120]}$ In 2014, W. C. MacMillan and co-workers accomplished the $\mathrm{C}-\mathrm{H}$ functionalization of benzyl ethers by using the radical generated from the PCET-promoted 


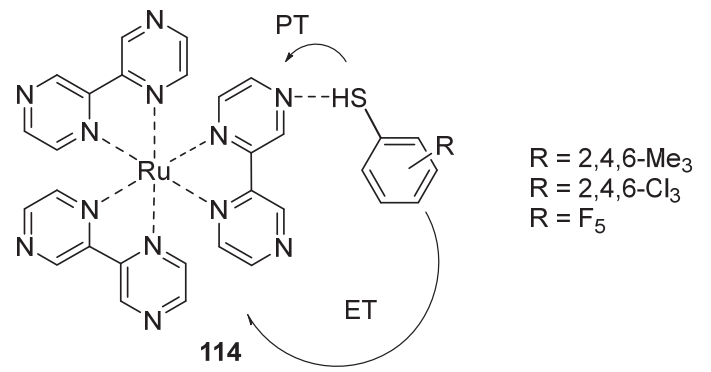

Scheme 47 PCET reactions between a photoexcited $\mathrm{Ru}(\mathrm{II})$ complex and thiophenols

breaking of $\mathrm{S}-\mathrm{H}$ bond (Scheme 48 ) ${ }^{[121]}$ This method leads to the efficient and direct synthesis of $\mathrm{C}-\mathrm{C}$ coupling products from a large range of ether and aryl components, and has mild reaction conditions.

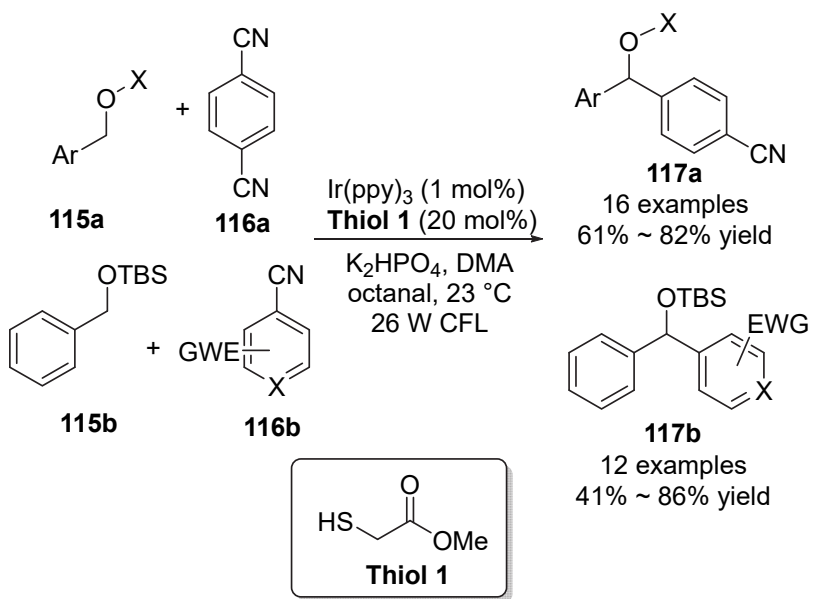

Proposed mechanism:

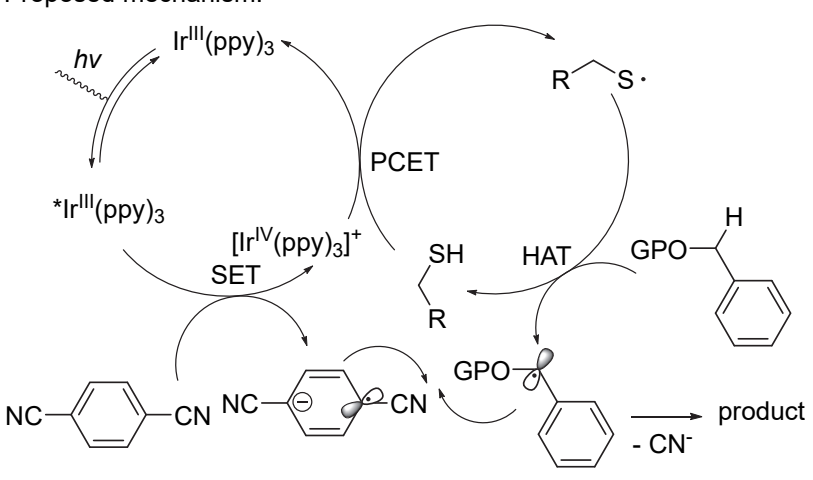

Scheme $48 \mathrm{C}-\mathrm{H}$ functionalization of benzyl ethers

With the similar conditions, they also reported another $\mathrm{C}-\mathrm{H}$ functionalization protocol in which ethers and imines are the coupling partners (Scheme 49). ${ }^{[122]}$ Through this strategy, a various of $\beta$-amino ether products could be attained in moderate to good yield.

Yoon's ${ }^{[123]}$ group reported a photocatalyst method for the efficient conjugation of thiols and alkenes (Scheme 50). This method uses $p$-toluidine as the redox mediator, and has a large substrate scope as well as the mild aqueous reaction conditions.

Rencently, in 2020, Wei-Min He and co-workers developed a cascade coupling and cyclization reactions of aro-

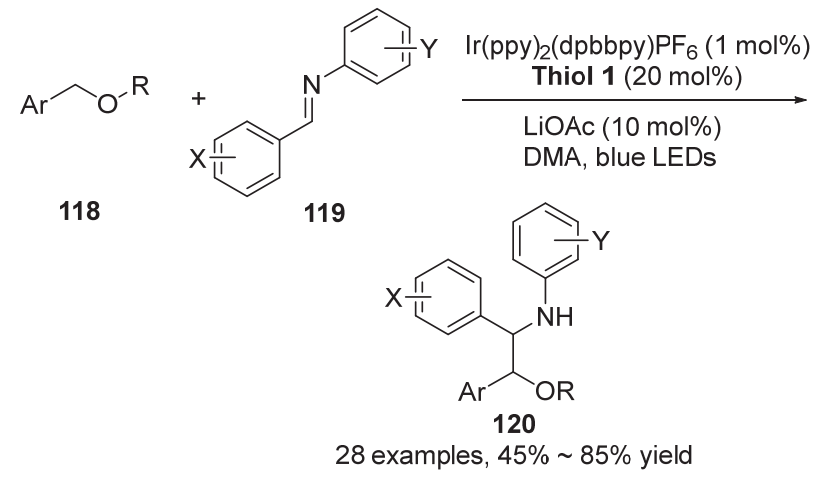

Scheme 49 Synthesis of $\beta$-amino ether from $\mathrm{C}-\mathrm{H}$ functionalization of ethers with imines

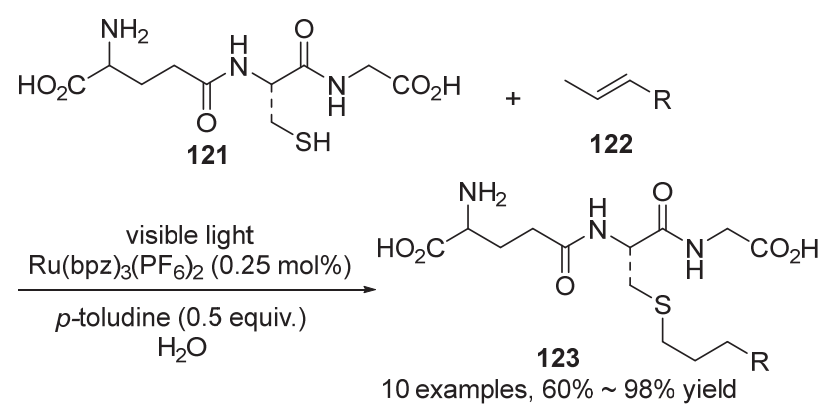

Scheme 50 Photocatalyst conjugation of thiols and alkenes

matic amines to form 2-aminobenzothiazoles (Scheme 51). The reactions are promoted by visible-light-initiated malic acid and without the requirement of photocatalyst. Ambient are employed as oxidant in this method. ${ }^{[124]}$

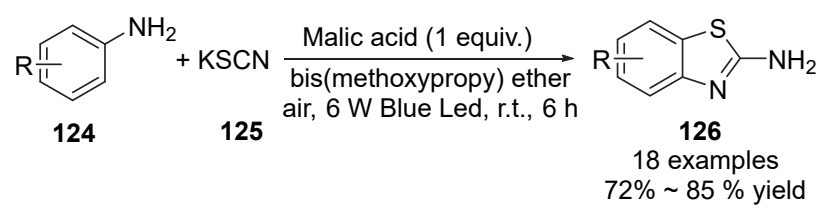

Proposed mechanism:

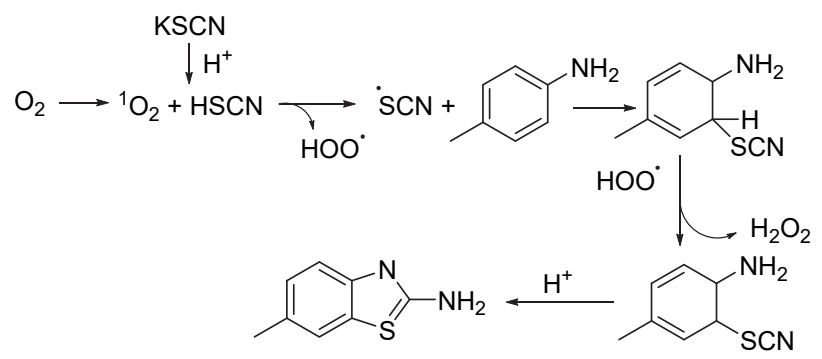

Scheme 51 Cascade coupling/cyclization reactions of aromatic amines

\subsection{Oxidative decarboxylation}

Oxidative decarboxylation is a brand new application of PCET processes in organic synthesis in recent years. ${ }^{[125-129]}$ Compared to traditional decarboxylation reactions, such as Barton decarboxylation, these PCET-involving decarboxylation reactions employ carboxylic acids instead of metal carboxylates or carboxylic ester as substrates, which leads to a better atom economy. In 2019 , Jiang's group ${ }^{[125]}$ re- 
ported asymmetric aerobic decarboxylative Povarov reactions, in which $N$-aryl $\alpha$-amino acids and methylenephthalimidines are substrates (Scheme 52). This method applied a dual catalyst system consists of photoredox DPZ and chiral phosphoric Brønsted acid C3, and without the requirement of metal reagents. In this work, the $\alpha$-aminoalkyl radical species would be generated via deprotonation, proton transfer, and decarboxylation, or called an oxidative decarboxylation, and followed by a further electron transfer.

This sort of $\alpha$-aminoalkyl radical species generated from an oxidative decarboxylation is further applied to other reactions. In the same year, Jiang's group ${ }^{[126]}$ developed a $[3+2]$ cycloaddition of $N$-aryl $\alpha$-amino acids with isoquinoline $N$-oxides via photoredox catalysis (Scheme 53). Similarly, an $\alpha$-aminoalkyl radical is generated from the
$N$-aryl $\alpha$-amino acids via PCET process, followed by a $[3+$ 2] cycloaddition in the presence of 1,3-dipoles to afford fundamental $N$-heterocyclic compounds.

Difunctionalization of alkenes also achieved in the presence of $N$-aryl $\alpha$-amino acids via analogous oxidative decarboxylation mechanism under visible light by Jiang's group, leading to the synthesis of isoxazolidines, which are important for their bioactivities and synthetic value, in up to $85 \%$ yield (Scheme 54$){ }^{[127]}$

Later, they successfully afforded a variety of chiral 3aminomethylene-3-substituted oxindoles with quaternary carbon stereocenters from 3-substituted 3-chlorooxindoles, also in the presence of $N$-aryl $\alpha$-amino acids, under the dual catalyst system containing photoredox DPZ and Brønsted acid catalyst SPINOL-CPA (Scheme 55). ${ }^{[128]}$

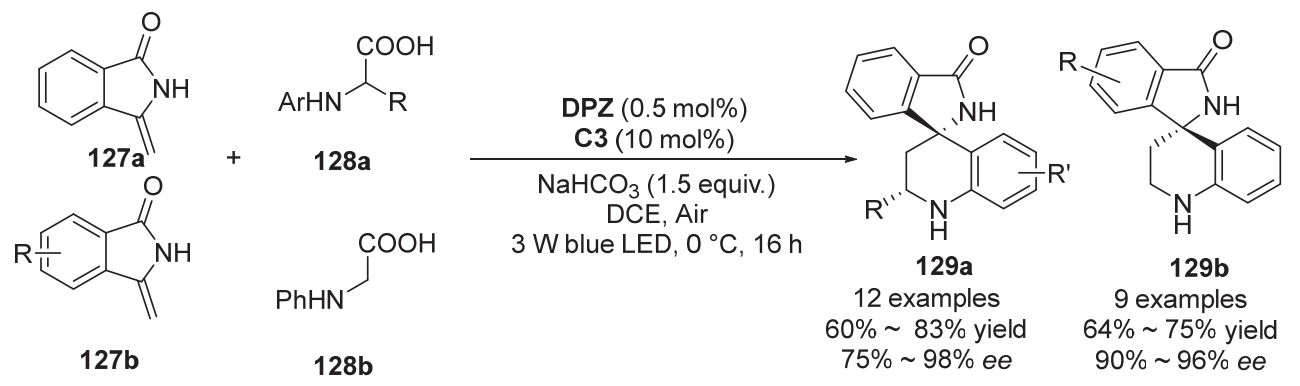

Proposed mechanism:

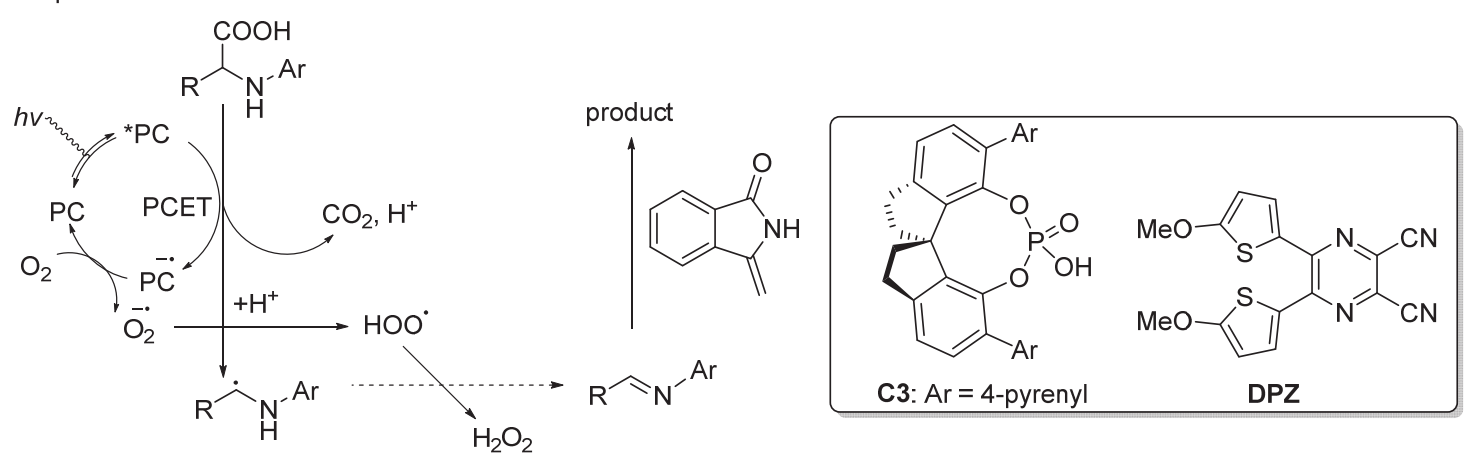

Scheme 52 Asymmetric aerobic decarboxylative Povarov reactions

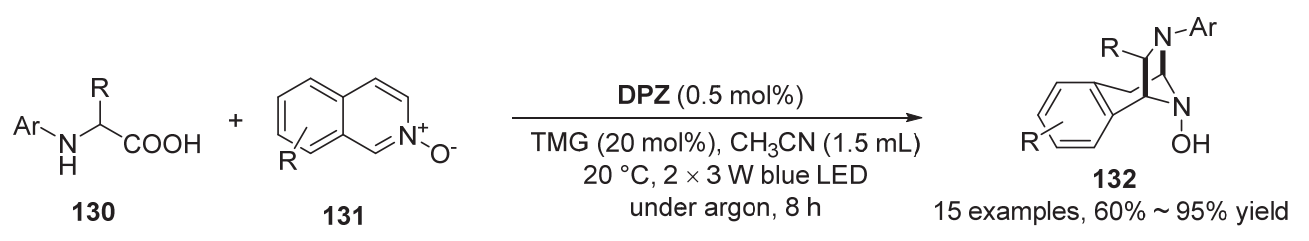

Scheme $53[3+2]$ cycloaddition of $N$-aryl $\alpha$-amino acids with isoquinoline $N$-oxides

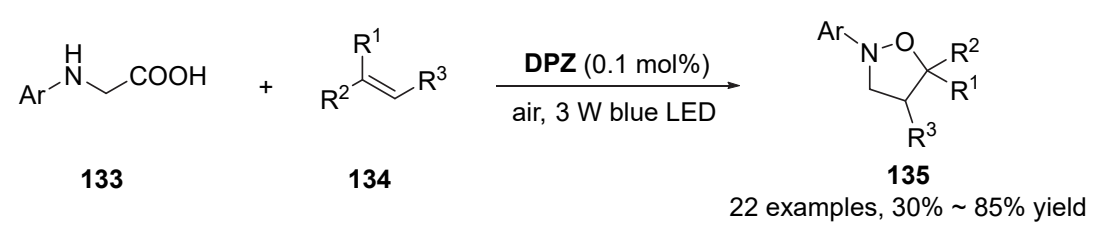

Scheme 54 Difunctionalization of alkenes in the presence of $N$-aryl $\alpha$-amino acids 
<smiles>[R]N1C(=[OH+])C([R])(Cl)c2ccccc21</smiles>

$136 a$ $137 a$

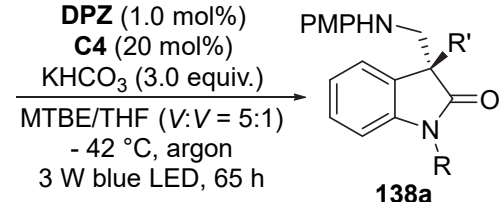

20 examples, $53 \% \sim 93 \%$ yield $80 \% \sim 98 \%$ ee<smiles>CN1C(=[OH+])C(Cl)(Cl)c2cc[R7]cc21</smiles>
$121 b$

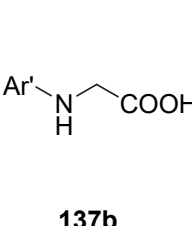

$137 b$

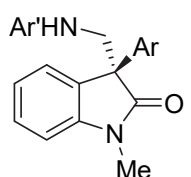

$138 \mathrm{~b}$

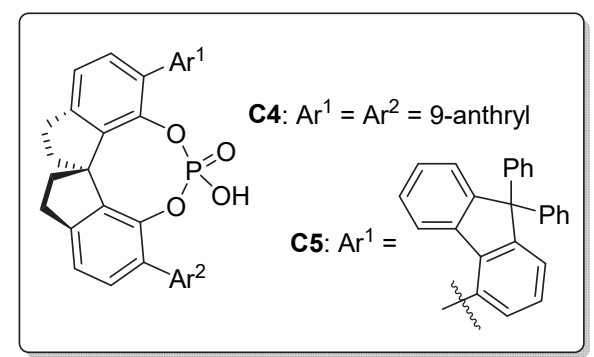

23 examples, 53\% 93\% yield $86 \% \sim 95 \%$ ee

Scheme 55 Synthesis of chiral 3-aminomethylene-3-substituted oxindoles with quaternary carbon stereocenters

\section{Reduction of $\mathrm{C}=\mathrm{Y}$ bond}

\section{1 $\mathrm{C}=\mathrm{C}$ bond}

Selective reduction of $\mathrm{C}=\mathrm{C}$ bond in $\alpha, \beta$-unsaturated carbonyl compounds can be accomplished by several ways, but all of them have their demerits. On the other hand, although reductive PCET has been employed for the reduction of alkenes, its selectivity is still not well-determined. To develop a simple, efficient method, in 2019, Sekar's group $^{[130]}$ developed a highly chemoselective reduction of $\alpha, \beta$-unsaturated ketones to saturated ketones through PCET under a transition-metal-free condition (Scheme 56). In the reduction of $\alpha, \beta$-unsaturated ketones, a proton transfers from formic acid mixture to the $\alpha, \beta$-unsaturated ketones substrates, and an electron transfers from xanthate also to the substrates.

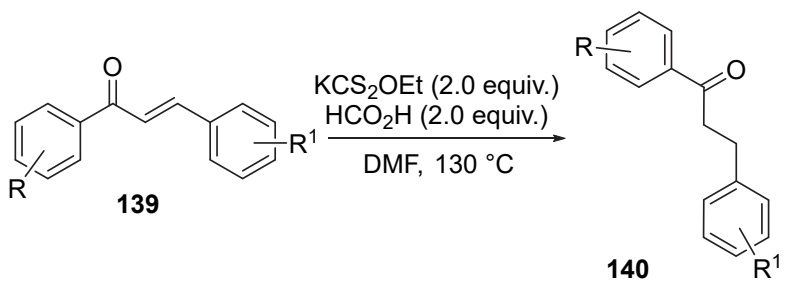

Proposed mechanism: 24 examples, $43 \% \sim 99 \%$ yield

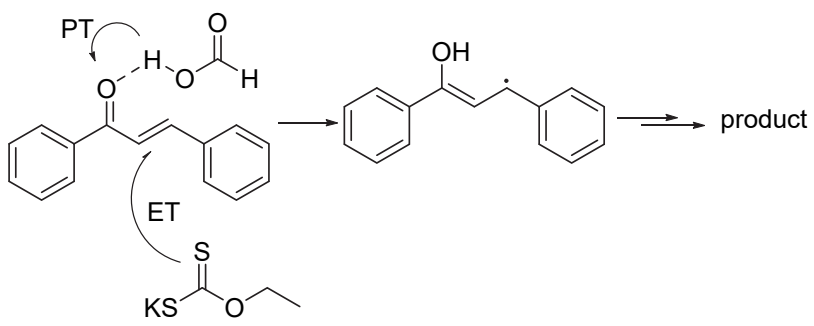

Scheme 56 Chemoselective reduction of $\alpha, \beta$-unsaturated ketones to saturated ketones

The reductive PCET of $\mathrm{C}=\mathrm{C}$ bonds in $\alpha, \beta$-unsaturated carbonyl compounds can also be followed by the formation of a new $\mathrm{C}-\mathrm{C}$ bond via a radical addition to another alkenes. In 2021, Jiang's group ${ }^{[131]}$ reported a novel strategy in order to access the enantioselective protonation that is triggered by the reductive cross coupling of olefin substrates (Scheme 57). Through this method, under the reaction conditions consist of cooperative photoredox and chiral hydrogen-bonding catalyst, in addition of a terminal reductant, a diverse range of enantioenriched azaarene products with tertiary stereocenters at their $\delta$-position, which are important structures commonly exist in many natural products, could be generated from the corresponding $\alpha$-branched vinylketones in both high yield and ee.

For electrochemical hydrogenation of activated hydrocarbons, these reactions often undergo a cathodic reduction of the activated olefins. Combining with the following protonation step, they can be considered as a stepwise PCET process. Compared to traditional approaches for the hydrogenation of unsaturated hydrocarbons, these electrochemical methods require no hydrogen gas, which makes them safer, less costly, and be of considerable value for the green chemistry. Several examples of the hydrogenation of activated olefins via electrochemical have been reported. One example was developed in 2019 by $\mathrm{Li}$ and co-workers, ${ }^{[132]}$ in which ammonia serves as the hydrogen source (Scheme 58). This metal-free electrochemical method leads to the hydrogenation of alkenes, alkynes, and ketones, and features a large scope of substrates. What's more, the employment of ammonia in an electrochemical system is safe and environmentally friendly.

Some old-school reactions may also proceed through PCET pathways. For instance, Birch reduction can be considered to undergo a stepwise PCET process. ${ }^{[133]}$ Reductive hydrogenation of unsaturated hydrocarbons via electrochemical Birch reactions has been existed for a long time. ${ }^{[134-138]}$ And recently in 2019, inspired by the development of lithium-ion battery materials, electrolytes and additives, Rodriguez and co-workers ${ }^{[139]}$ reported a scalable and safe method for the synthesis of a large range of pharmaceutical building blocks by electrochemical Birch reduction (Scheme 59). Their further study demonstrated the practical potential of this method in other metal-type 


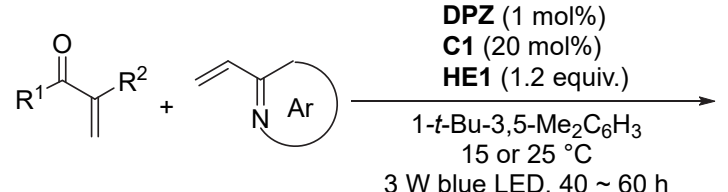

142

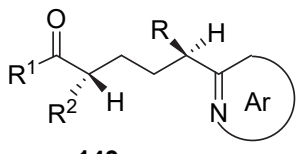

143

57 examples, $35 \% \sim 95 \%$ yield $62 \% \sim 99 \%$ ee

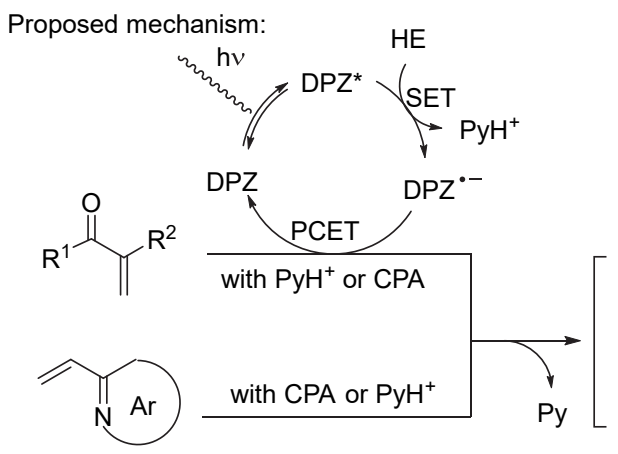

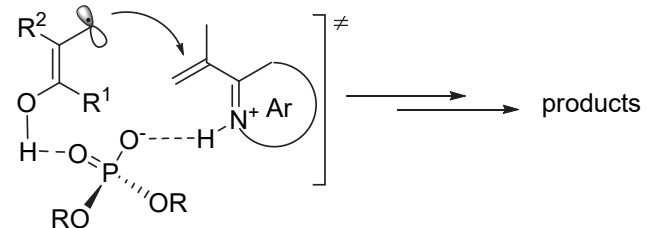

Scheme 57 Enantioselective protonation triggered by the reductive cross coupling of olefin substrates

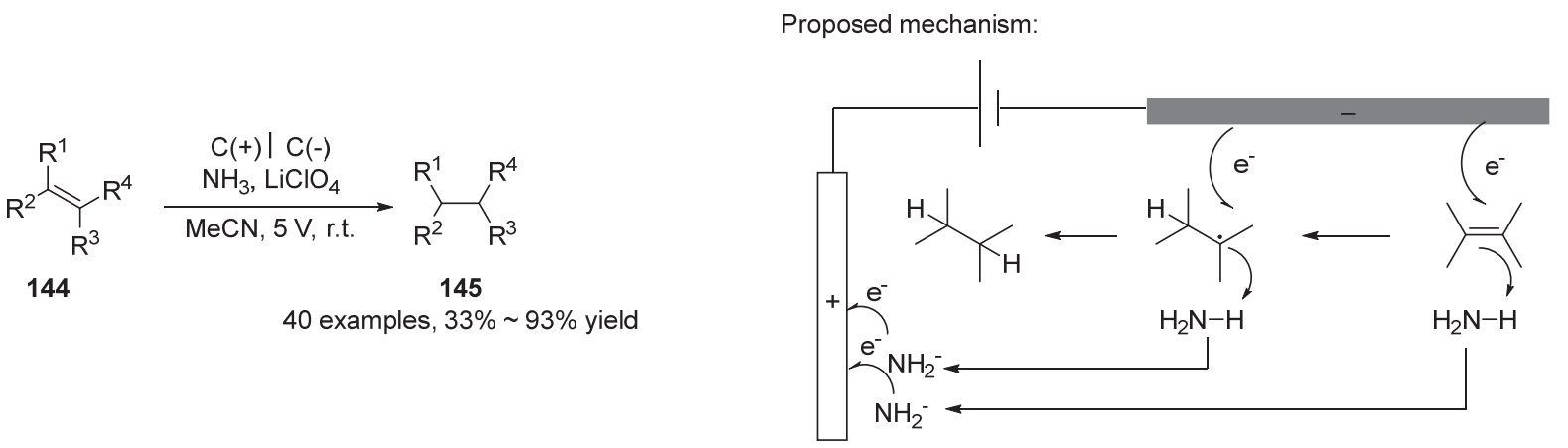

Scheme 58 Electrochemical hydrogenation of activated olefins

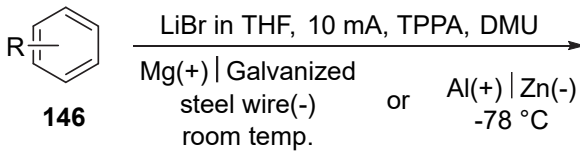

Proposed mechanism:

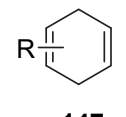

147

34 examples, $39 \% \sim 85 \%$ yield

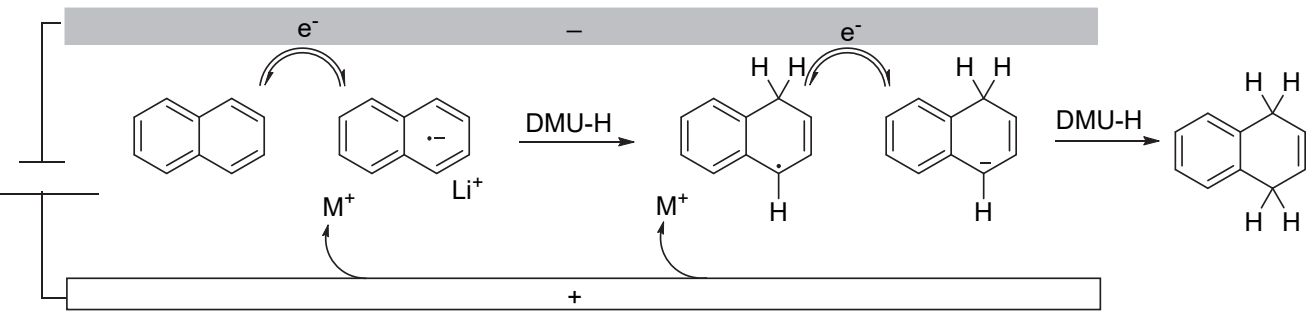

Other electroreductive transformations:

${ }_{T_{n}}^{\mathrm{R}^{1} \widehat{\mathrm{X}} \widehat{\mathrm{R}^{1}} \text { or }}$ or ${ }_{\mathrm{R}^{1}}^{\mathrm{148}}$

$148 b$

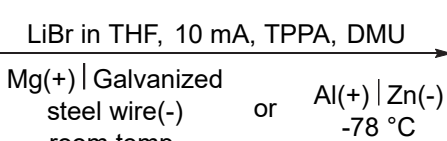

room temp.

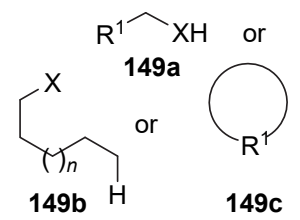

149c

Scheme 59 Hydrogenation via electrochemical Birch reduction 
reductive transformations, such as McMurry couplings and so on.

In 2020, Huang and co-workers ${ }^{[140]}$ reported a chemoselective method for the hydrogenation of unsaturated $\mathrm{C}-\mathrm{C}$ bond (Scheme 60). This method proceeds under mild metaland external-reductant-free conditions, and can deliver a large range of reductive products from substituted alkenes, unsaturated acids, esters, and amides. Notably, both DMSO and $\mathrm{H}_{2} \mathrm{O}$ can serve as the hydrogen source.

In the same year, with $\mathrm{D}_{2} \mathrm{O}$ as hydrogen source, Cheng and co-workers ${ }^{[141]}$ also reported a reductant- and catalystfree electrochemical protocol for the reductive hydrogenation of $\alpha, \beta$-unsaturated carbonyl compounds (Scheme 61). This deuteration reaction can afford the hydrogenated products in moderate to good yield, and can also achieve the reduction of triple $\mathrm{C}-\mathrm{C}$ bond to single $\mathrm{C}-\mathrm{C}$ bond. Through this method, a variety of deuterated pharmaceutical compounds could be prepared.

\section{$4.2 \mathrm{C}=\mathrm{N}$ bond}

Compounds with carbon-heteroatom double bonds such as $\mathrm{C}=\mathrm{O}$ bond (ketones and aldehydes) and $\mathrm{C}=\mathrm{N}$ bond (imines) are important in organic synthesis for the formation of new $\mathrm{C}-\mathrm{C}$ bond, as well as the preparation of some synthetically intermediates and valuable building blocks including alcohols and amines. For reduction of $\mathrm{C}=$ $\mathrm{N}$ bond, several novel strategies have been reported via a PCET mechanism.

In 2018, Dixon's group ${ }^{[142]}$ described the synthesis of 1,3-diamines from in situ-generated imines and dehydroalanine derivatives with a visible-light-mediated photocatalytic and umpolung method (Scheme 62). Before their
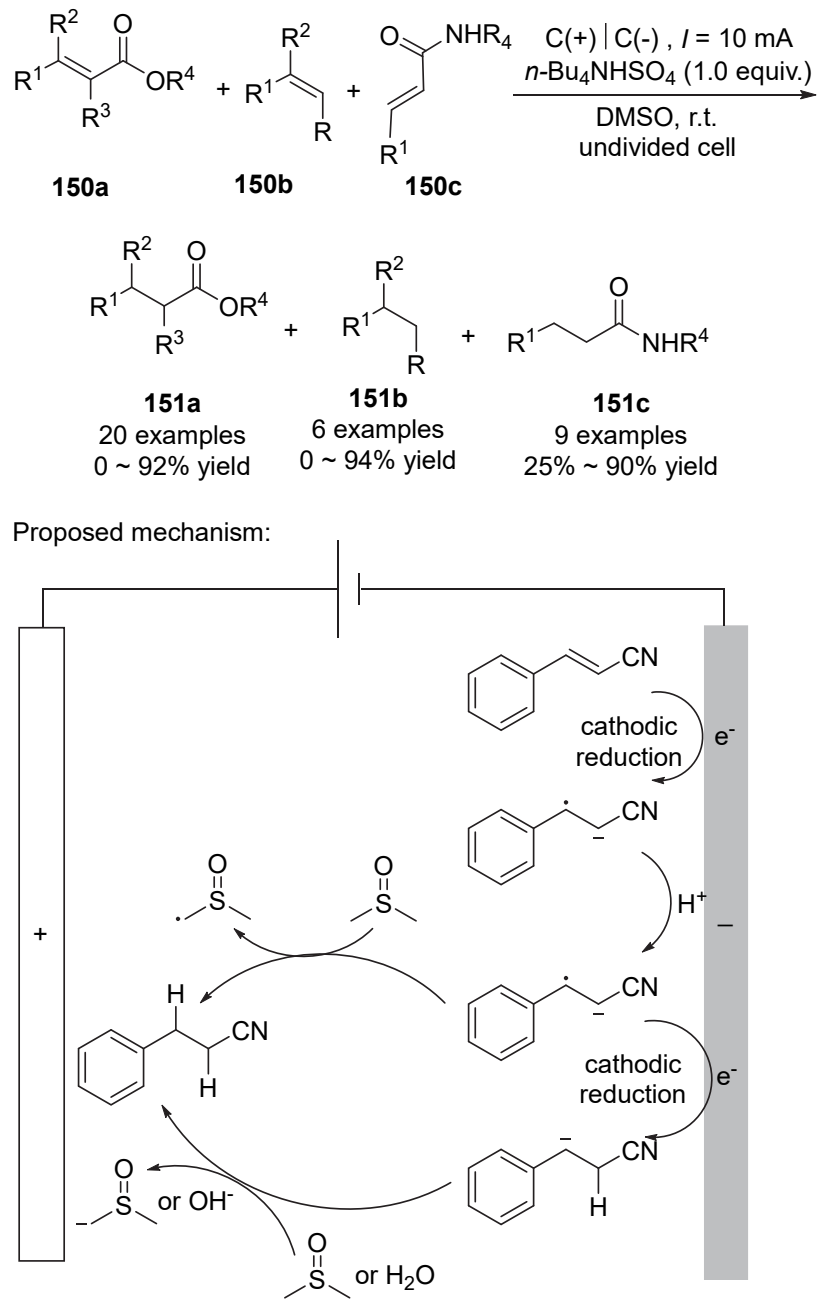

Scheme 60 Chemoselective hydrogenation of unsaturated C$\mathrm{C}$ bond

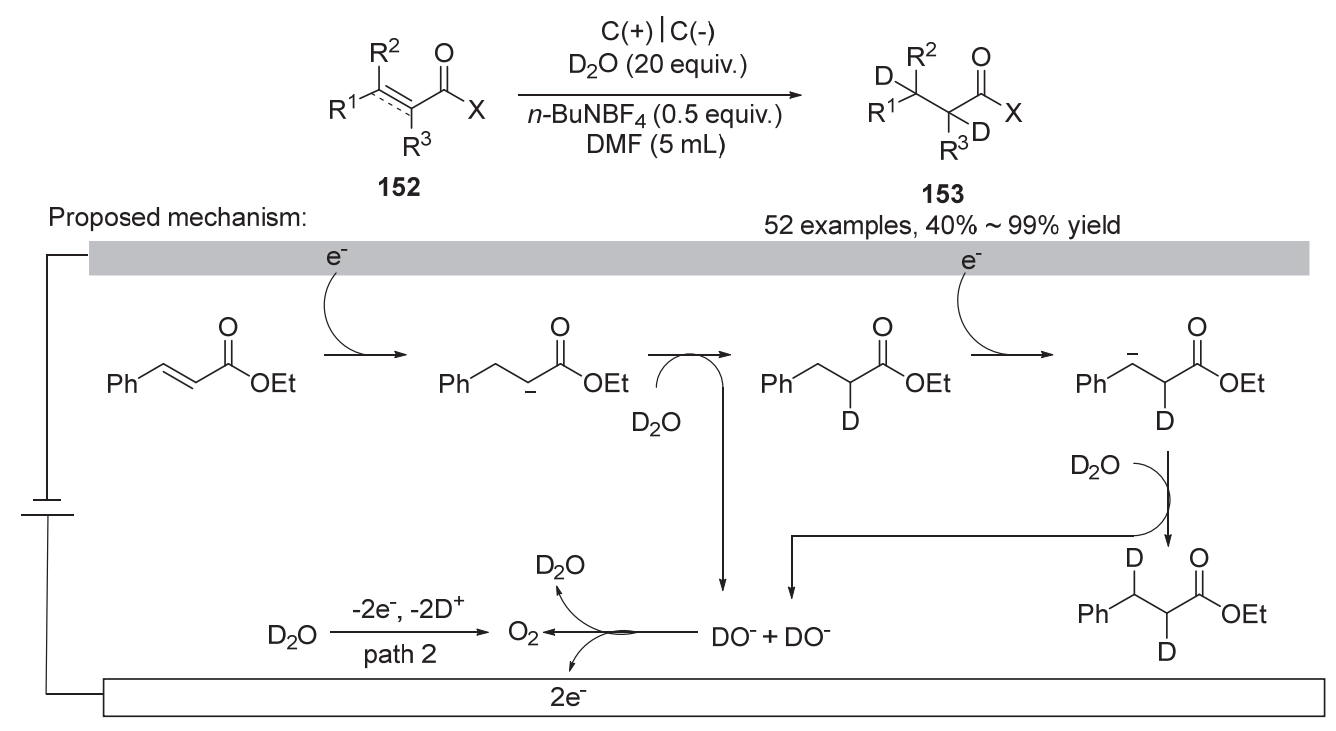

Scheme 61 Electrochemical reductive hydrogenation of $\alpha, \beta$-unsaturated carbonyl compounds 


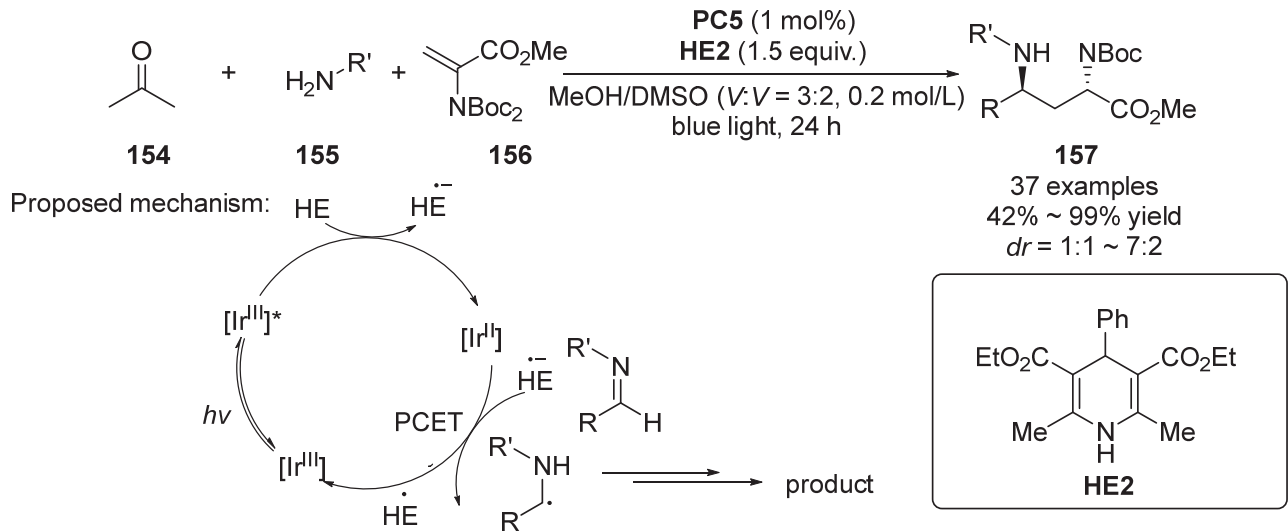

Scheme 62 Synthesis of 1,3-diamines with a visible-light-mediated photocatalytic and umpolung method

work, development of visible-light photoredox catalysis had been made enabled by the in-situ generated free-radical intermediates, which led to a various of novel methods for the $\mathrm{C}-\mathrm{C}$ and $\mathrm{C}-\mathrm{X}$ bond-forming. On the other hand, significant achievements of umpolung transformations in coupling reactions had been made, among which the reductive coupling of imine and electrophile via an $\alpha$-amino radical is one of the most notable. Combining the two strategies, with Hantzsch ester (HE2) as H-atom donors, $\left[\operatorname{Ir}\left(\mathrm{dF}\left(\mathrm{CF}_{3}\right) \text { ppy }\right)_{2}(\mathrm{dtbbpy})\right]^{+}\left[\mathrm{PF}_{6}\right]^{-}$as photocatalyst and $\mathrm{MeOH} / \mathrm{DMSO}(3: 2, \quad V: V)$ as solvent, they achieved a large aldehyde and aniline substrates scope of the synthesis of 1,3-diamines. In their previous reports, they proposed that partially oxidized Hantzsch esters can donate a proton in PCET mechanisms, which achieve the reduction of $\mathrm{C}=\mathrm{N}$ bond by the iridium species.

And immediately after that, in 2019, they reported a general synthetic platform for the construction of primary $\alpha$-tertiary amines in quinone mediator, with primary a-branched amine as starting materials (Scheme 63). ${ }^{[143]}$ In this method, an imine structure is formed by the amine substrates in the presence of quinone derivatives. With the polarity reverse enabled by the photoredox catalysis according to their previous study described above, they found a new reactivity for this synthetic platform. As a result, a PCET reduction of imine generating a nucleophilic $\alpha$-amino radical which can be intercepted by electrophiles was employed, and this could expand the capability of their method.

Methods for the direct synthesis of primary amines with substituted quaternary $\alpha$-carbon were rare. In 2020, Rovis' group $^{[144]}$ developed a method coupling readily available $O$-benzoyl oximes (method A) or iminium chloride (method B) with cyanoarenes to achieve primary hindered amines with fully substituted $\alpha$-carbons by utilizing photoredox catalysis (Scheme 64). Besides, they also accomplished the synthesis of $\alpha$-trifluoromethyl amines through this method. This method provides a rapid way for the formation of fully $\alpha$-substituted primary amines without the requirement for subsequent deprotection.

Concurrently, they also achieved the synthesis of both hindered primary and secondary amines through an opera-<smiles>[R]C([R16])=Nc1ccc(C([R16])(C)C)cc1O</smiles>

(1) PC5 (1 mol \%), HEH (1.5 equiv.), DMSO, $16 \mathrm{~h}$

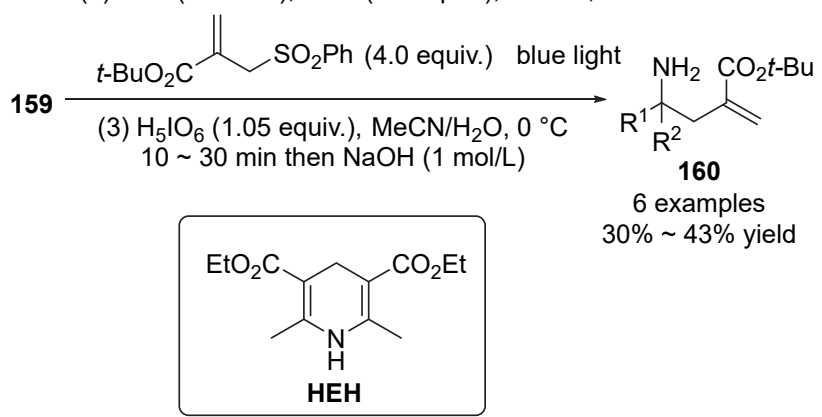

Scheme 63 Synthesis of primary $\alpha$-tertiary amines in quinone mediator

method A

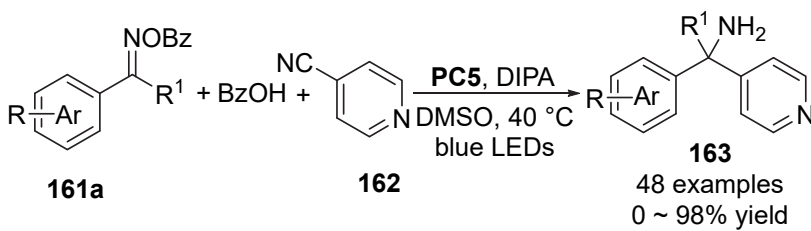

method B

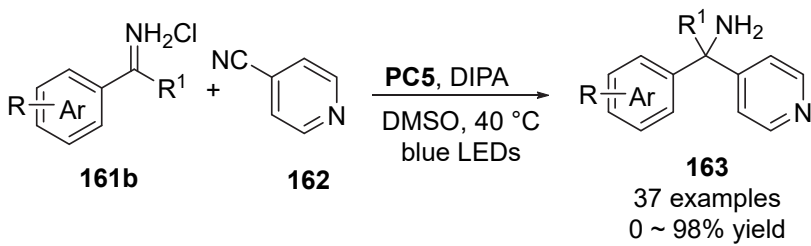

Scheme 64 Synthesis of primary hindered amines with fully substituted $\alpha$-carbons by utilizing photoredox catalysis

tionally simple electrochemical method via PCET mechanism using iminium chloride as starting material. Optimized conditions consists of $\mathrm{Et}_{4} \mathrm{NCl}$ as optimal electrolyte, DMSO 
as solvent, $\mathrm{Zn}$ as cathode and graphite as anode (Scheme $65) .{ }^{[145]}$ The reaction path ways proceed through a PCET process followed by a heteroradical-radical coupling, after that there would be a re-aromatization to generate products.

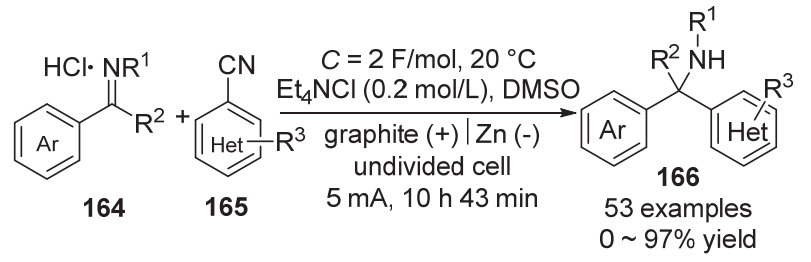

Scheme 65 Synthesis of both hindered primary and secondary amines

\section{3 $\mathrm{C}=\mathrm{O}$ bond}

Ketyls are intermediates that are of great importance for numerous important bond-forming and bond-breaking processes, and they are generally derived from one-electron reduction of carbonyl compounds, which require strong negative potentials, resulting to the limitation on the scope of redox partners. In 2013, Knowles' group ${ }^{[41]}$ reported a catalytic protocol for ketyl-olefin coupling to achieve the synthesis of lactones, in which concerted PCET was proposed to be the operative mechanism for the formation of ketyl radicals (Scheme 66).

In 2016, based on the previous works we have described above, Knowles' group considered concerted PCET as an effective synthesis platform for the asymmetric catalysis of radical reactions, and the further development had been made by introducing a pre-equilibrium hydrogen-bonding event into this platform, leading to new reactivity and a lager range of application (Scheme 67). ${ }^{[7]}$ By using similar reaction conditions to the PCET-mediated ketyl-olefin coupling described above, they were able to accomplish the synthesis for a wide range of cis-amino alcohol products in good to excellent yields and enantioselectivities.

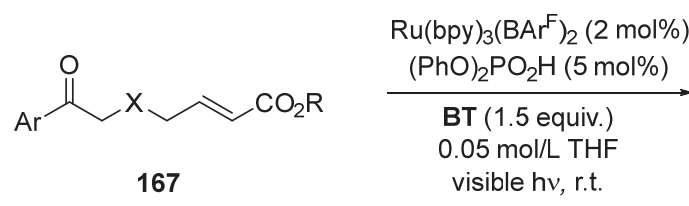

Proposed mechanism:

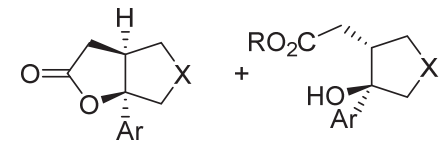

$$
\text { cis 168a trans 168b }
$$

10 examples, $64 \% \sim 96 \%$ yield, cis:trans $=1.2: 1 \sim 1: 0$
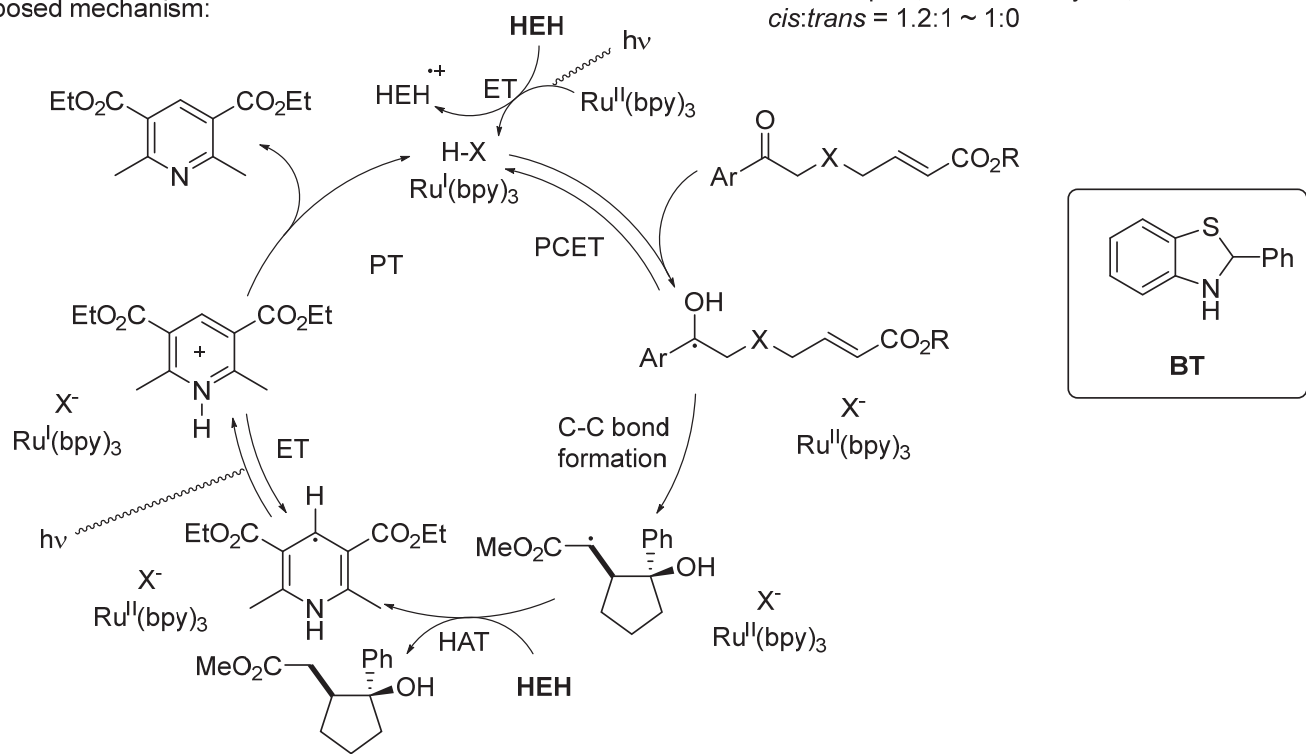

Scheme 66 Synthesis of lactones by ketyl-olefin coupling<smiles>[X]CCC=NN(C)CCC(=O)c1ccccc1</smiles>

169

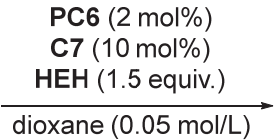

r.t., blue LEDs

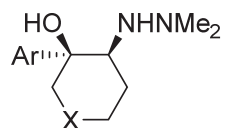

170

8 examples $58 \% \sim 96 \%$ yield, $77 \% \sim 95 \%$ ee

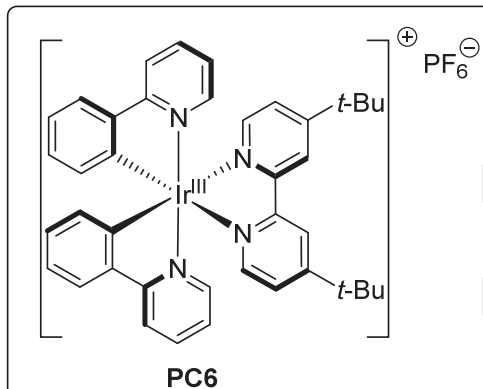

$\mathrm{PF}_{6}^{\ominus}$

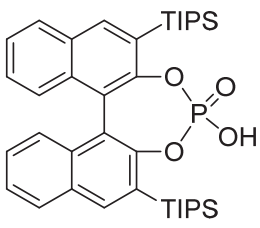

C7

Scheme 67 Synthesis for $c i s$-amino alcohols via concerted PCET 
In 2017, Xia and co-workers ${ }^{[146]}$ reported the first direct arylation for carbonyl/iminyl derivatives enabled by visible-light-mediated radical-radical cross-coupling methods, which lead to the synthesis of a large range of chemical compounds such as secondary/tertiary alcohols and amines (Scheme 68). They described the reduction of both $\mathrm{C}=\mathrm{O}$ bond and $\mathrm{C}=\mathrm{N}$ bond via PCET processes, and accessed the alcohol and amine products, respectively. They launched their initial work by using benzaldehyde as model substrate to form the target alcohol product under the reaction conditions of $\left[\operatorname{Ir}(\mathrm{ppy})_{2}(\mathrm{dtbbpy})\right]^{+} \mathrm{PF}_{6}^{-}$as photocatalyst with the presence of triethylamine in $\mathrm{MeOH}$. To extend the scope of their method, they further chose aromatic imines as new substrates to ensure the compatibility of this methology, and found that a various of imine derivatives were enabled to form corresponding products under the same reaction conditions.

In 2019, Jiang's group ${ }^{[147]}$ reported a method for the formation of $\gamma$-functionalized vinylpyridines by catalytic addition of prochiral radicals (Scheme 69). This method is operated under conditions including photoredox, asymmetric catalysis and visible light with a large scope, high yields and stereoselectivities, and the reduction can realize not only $\mathrm{C}=\mathrm{O}$ bond in aldehydes and ketones, but also $\mathrm{C}=\mathrm{N}$ bond in imines. According to their mechanism studies via control experiments and DFT calculations, it is suggested that there would be two plausible ways, both of which undergo a PCET process after the initiation of the photoredox catalytic cycle (Scheme 70).

Reduction of $\mathrm{C}=\mathrm{O}$ bond can also be achieved through electrochemical ways. In 2019, Xia and co-workers ${ }^{[148]}$ reported an electrochemical method for the 1,4-hydrogenation of $\alpha, \beta$-unsaturated ketones (Scheme 71). Ammonium chloride $\left(\mathrm{NH}_{4} \mathrm{Cl}\right)$ is the only additive in this system, which is used as the hydrogen source. In addition, methanol is also applied as the hydrogen source as well as the organic solvent. This user-friendly method was proven to be suitable for diverse substrates, and can be operated under mild reaction conditions.

\section{New frontiers of PCET theory and its application}

There are several new types of PCET reactions which both organic chemists and physical chemists are intrigued in. First, the inner-sphere PCET involving an inner-sphere electron transfer coupled with a proton transfer is a new emerging type of PCET which has been successfully applied in some organic reactions. In 2017, Das and coworkers developed a cobalt-catalyzed ortho-selective nitration of aromatic $\mathrm{C}\left(\mathrm{sp}^{2}\right)-\mathrm{H}$ bond in the presence of chelation-assisted vicinal diamine directing groups on the aromatic rings, and the reaction pathway involves an inner-sphere electron transfer and intramolecular proton transfer to generate the intermediate (179b) from the $\mathrm{Co}(\mathrm{III})$ species (179a) (Scheme 72). ${ }^{[149]}$ This intermediate leads to the product of a various of ortho-nitro derivatives $\mathbf{1 8 0}$
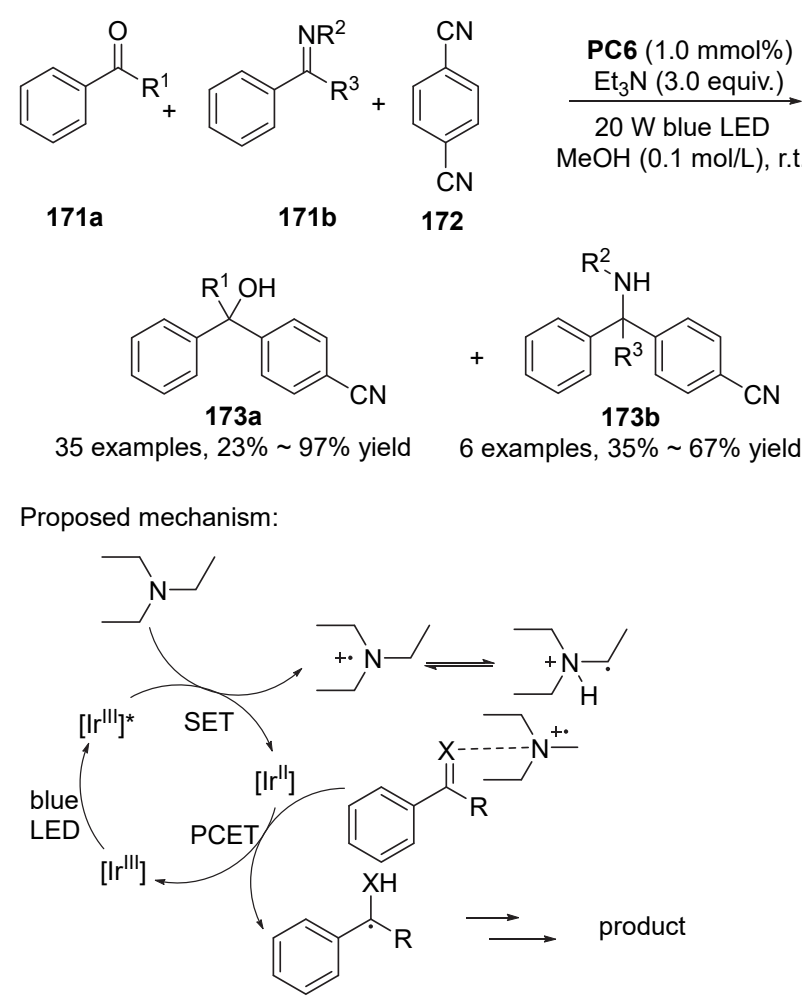

Scheme 68 Arylation for carbonyl/iminyl derivatives enabled by visible-light-mediated cross-coupling methods

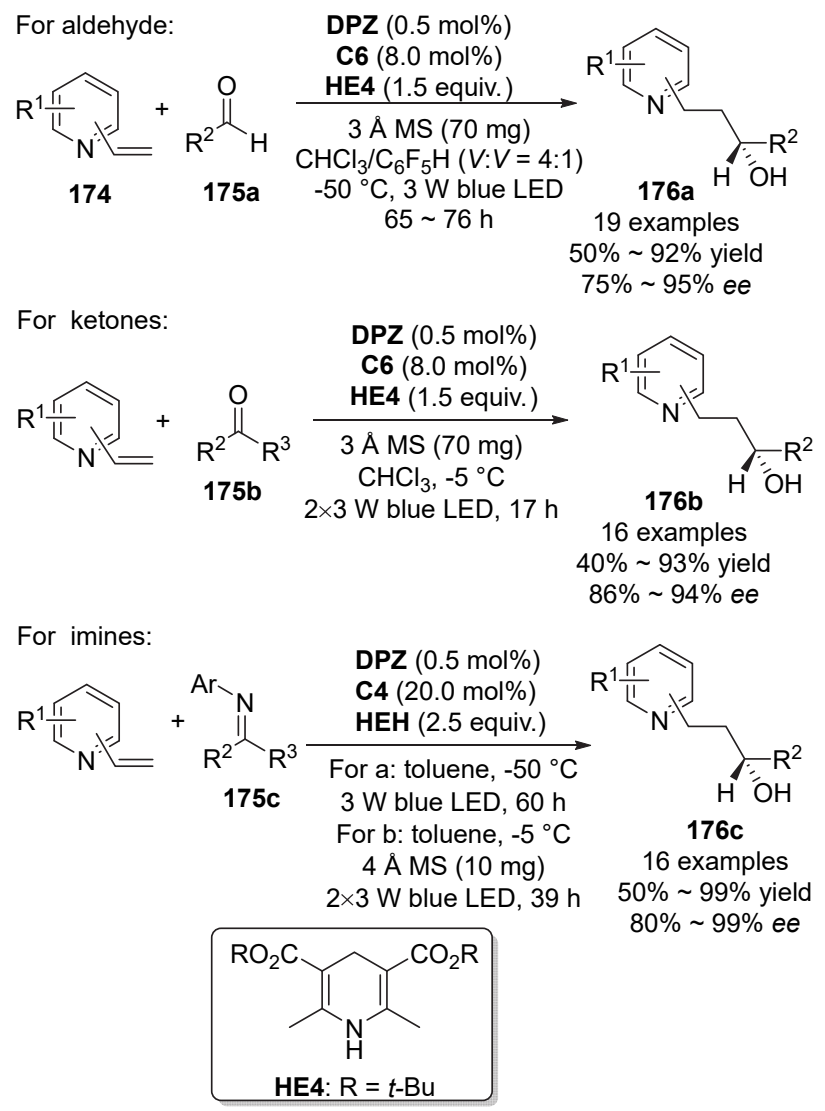

Scheme 69 Formation of $\gamma$-functionalized vinylpyridines by catalytic addition of prochiral radicals 


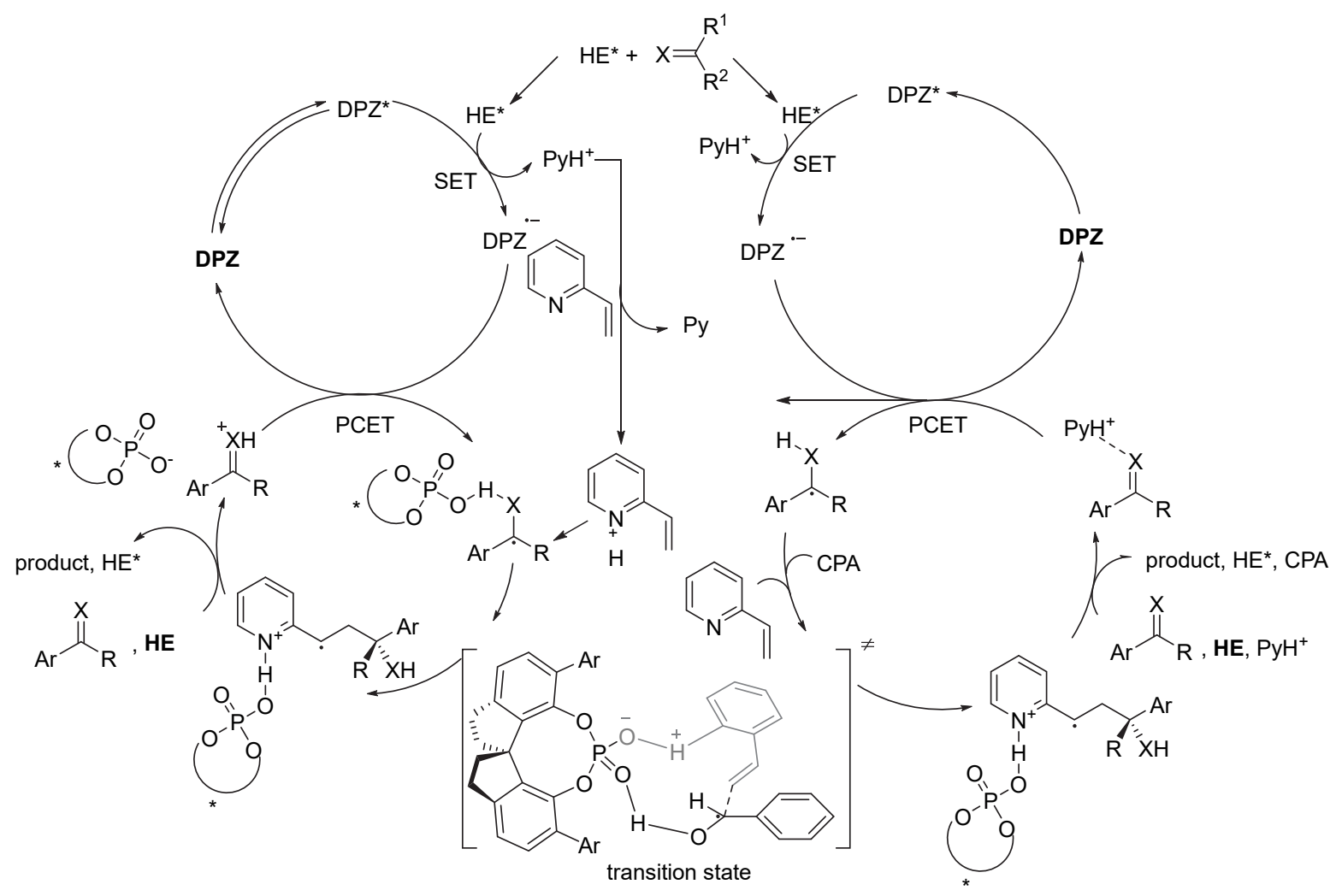

Scheme 70 Two plausible mechanisms for the formation of $\gamma$-functionalized vinylpyridines

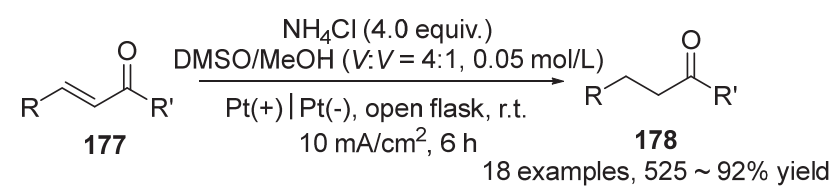

Proposed mechanism:

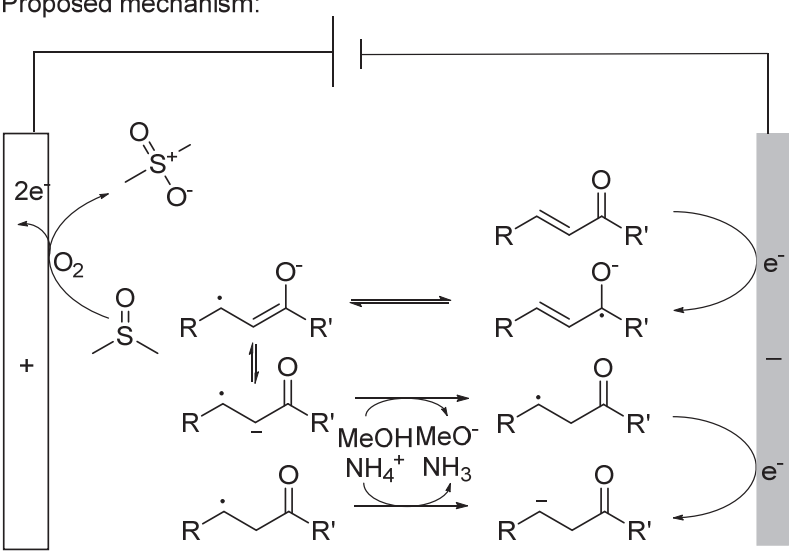

Scheme 71 Electrochemical 1,4-hydrogenation of $\alpha, \beta$-unsaturated ketones

with good functional group tolerance and regioselectivities.

In 2019, Liu's group ${ }^{[150]}$ reported a $\mathrm{Cu}(\mathrm{I})$-catalyzed enantioselective phosphinocyanation of styrenes via cascade radical relay strategy (Scheme 73 ). They proposed that $t$-BuOOSiMe $\mathrm{Bu}_{3}$ serves as a radical initiator to trigger $t$-butoxy radical production by inner-sphere PCET reaction with $\mathrm{L}^{*} \mathrm{Cu}(\mathrm{I})$ species, and then the phosphinoyl and benzyl radical are formed sequentially. This strategy offers the effective synthesis of alkylnitriles in both good yields and enatioselectivities under mild reaction conditions. Because of this new type of PCET process, new questions have arisen both experimentally and theoretically to the chemists, waiting for them to explore the fundamental rules. ${ }^{[6]}$

In 2018, Zuo and co-workers reported the development of photocatalytic $\mathrm{C}-\mathrm{H}$ functionalization, including amination, alkylation and arylation, for methane, ethane and alkanes. This method applies easily available cerium salts as the photocatalyst, leading to the high catalytic efficiency as well as good chemoselectivity of the reactions. The reaction pathways involve a ligand-to-metal charge transfer (LMCT) excitation, which is a photoexcitation manifold and may generate a hydroxyl radical serving as the HAT catalyst, enabling the functionalization of $\mathrm{C}-\mathrm{H}$ bond (Scheme 74).

The excited-state PCET (ES-PCET) is another important topic on PCET mechanism which is attracting chemists' attentions in recent years (Figure 4). ${ }^{[151-166]}$ The experimental studies on ES-PCET have been published by the leading groups like Ming-Tian Zhang ${ }^{[151]}$ and others, however, the ES-PCET theory is still under developing right now. ES-PCET can directly use the energy of photons to drive the reaction, and electrons and protons transfer directly in the excited state of substrate, which greatly improves the energy conversion efficiency. ${ }^{[165]}$ The design and development of more ES-PCET reactions will help to enrich the excited state proton-coupled electron transfer theory. In 2019, with the help of ES-PCET, Hammarström, Mayer and 
<smiles>[18F]c1ccccc1Nc1ccccn1</smiles>

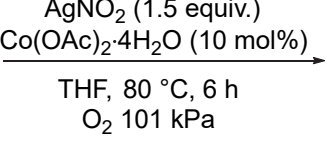<smiles>O=[N+]([O-])c1ccccc1Nc1ccccn1</smiles>

180

24 examples, $68 \% \sim 92 \%$

(1) $\mathrm{Pd} / \mathrm{C}, \mathrm{H}_{2}(101 \mathrm{kPa}), \mathrm{HCl}$ i-PrOH, r.t., $16 \mathrm{~h}$

(2) $\mathrm{NH}_{2} \mathrm{NH}_{2} \cdot \mathrm{H}_{2} \mathrm{O}, \mathrm{AcOH}$ $\mathrm{EtOH}, 120^{\circ} \mathrm{C}, 30 \mathrm{~min}$<smiles>Nc1ccccc1N</smiles>

182

Proposed mechanism:

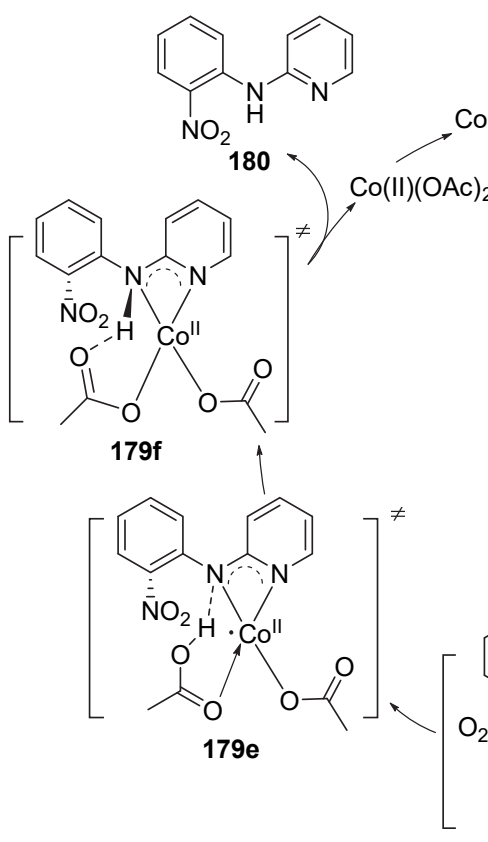

180 $\mathrm{H}_{2}$<smiles>c1ccc(Nc2ccccn2)cc1</smiles>
$\mathrm{Co}(\mathrm{III})(\mathrm{OAc})_{2} \mathrm{NO}_{2}$

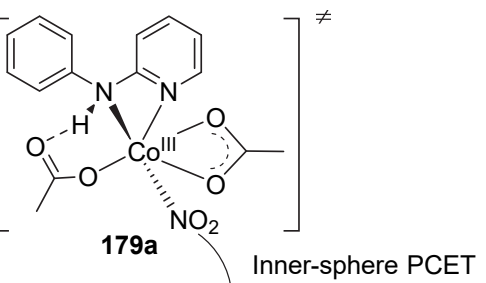

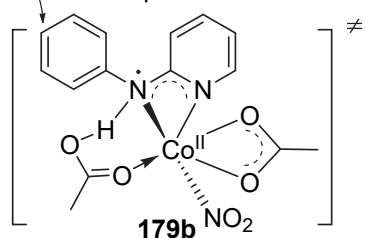

$179 b$<smiles>CC(C)C(=O)O</smiles>

$\longrightarrow \mathrm{AcOH}$

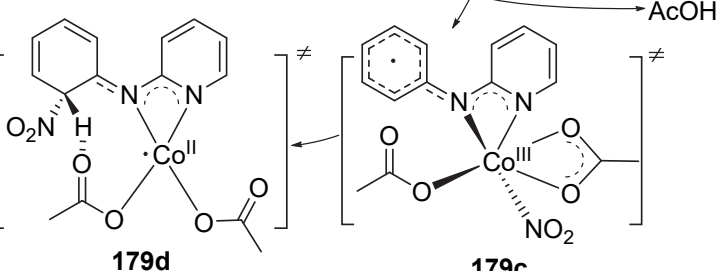

$179 c$

Scheme 72 Cobalt-catalyzed ortho-specific regioselective nitration of aromatic $\mathrm{C}\left(\mathrm{sp}^{2}\right)-\mathrm{H}$ bond

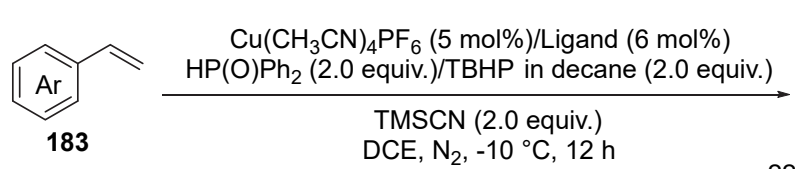

Proposed mechanism:

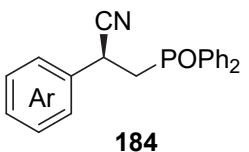

22 examples, $60 \% \sim 96 \%$ yield $76 \% \sim 97 \%$ ee

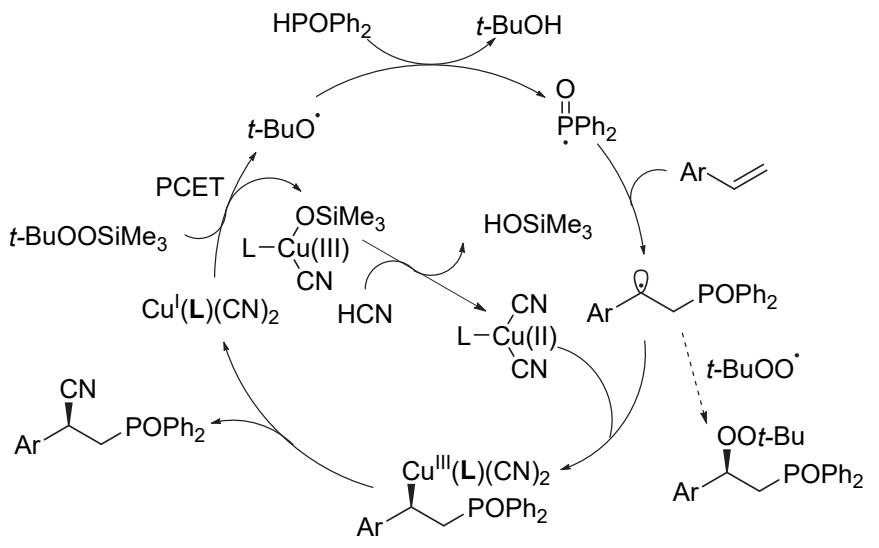

Scheme $73 \mathrm{Cu}(\mathrm{I})$-catalyzed enantioselective phosphinocyanation of styrenes 


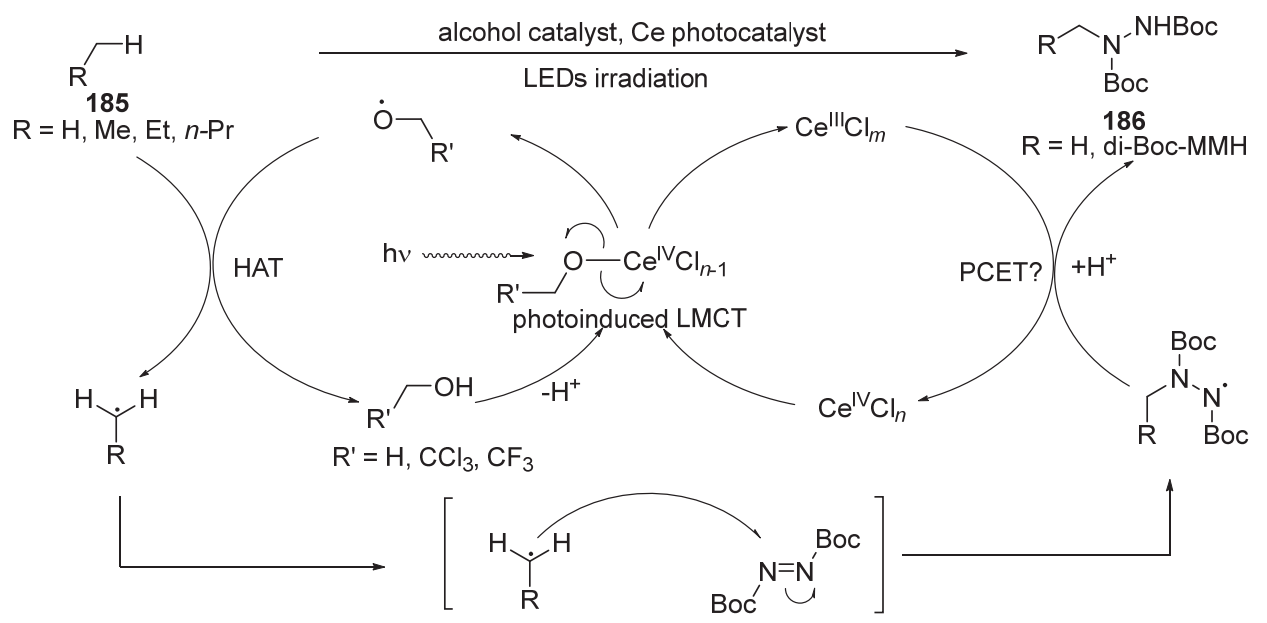

Scheme 74 Proposed mechanism for the cerium-catalyzed $\mathrm{C}-\mathrm{H}$ functionalization

(A)

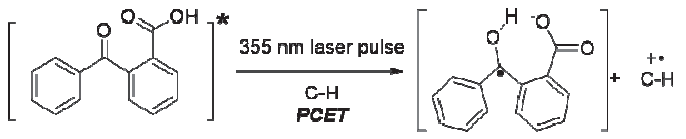

(B)

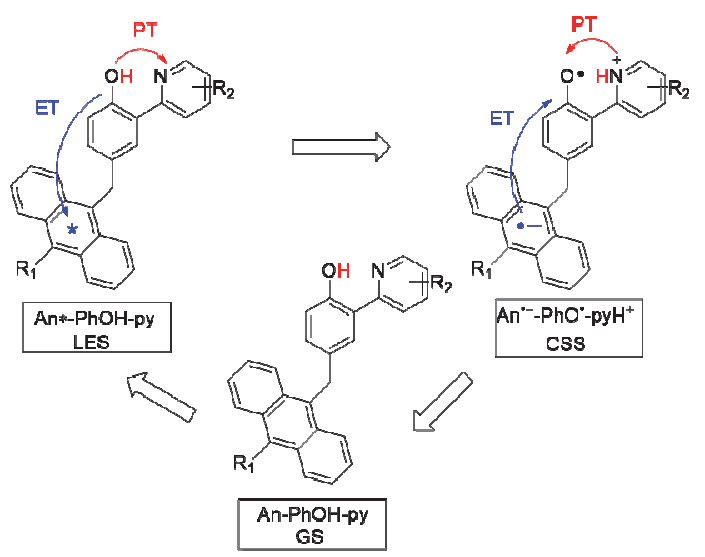

(C)

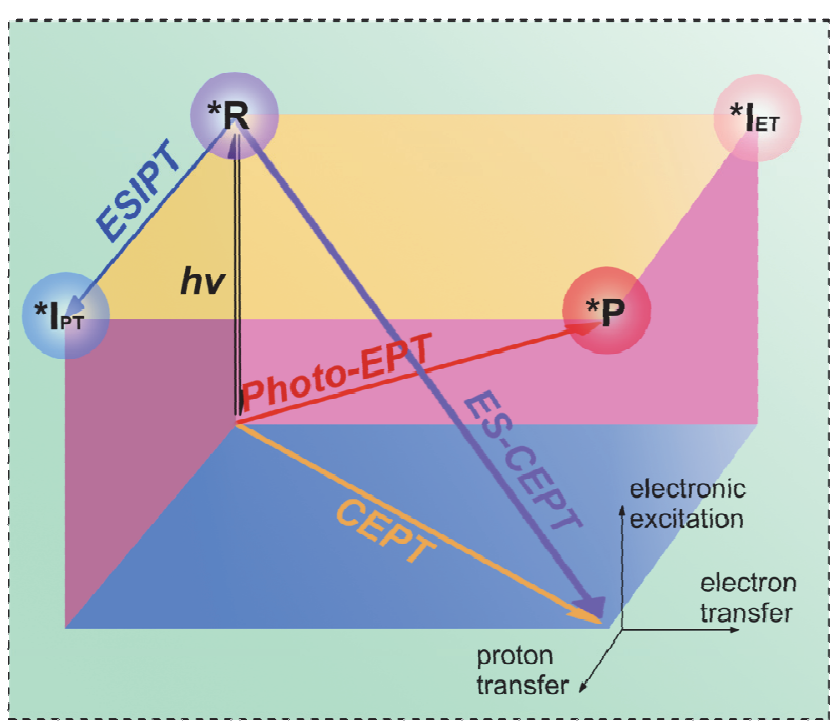

Figure 4 (A) A representative work of ES-PCET; ${ }^{[151]}$ (B) ES-PCET in the research of the Marcus inverted region; ${ }^{[166]}(\mathrm{C})$ electronic ground- and excited-state square schemes of PCET (redrawn from J. Am. Chem. Soc. 2021, 143, 560. ${ }^{[6]}$ )

Hammes-Schiffer jointly reported experimental evidence that the Marcus inverted region exists in the PCET reaction. ${ }^{[166]}$ After excitation, the excited state anthracene (LES) undergoes ES-PCET to get a charge separated state (CSS) and then goes through charge recombination back to ground state (GS). By changing the substituents of anthracene-phenol-pyridine triads and solvents, in the process of charge recombination, it is observed that the more favorable the thermodynamics of the reaction is, the lower the reaction rate will be, which is the behavior of the inverted region in Marcus theory. Further study of ES-PCET will help to understand the relationship between electron and proton transfer, and it may not only provide new ideas and methods for synthetic chemistry, but also promote the understanding of photosynthesis in biological systems so as to provide inspiration for efficient use of solar energy.

$\mathrm{Wu}$ and Tung's group recently reported a series of reac- tions which achieve the $\mathrm{C}-\mathrm{X}$ coupling with hydrogen evolution via electrocatalyst or photocatalyst methods. Through this kind of reactions (Scheme 75), they achieved $\mathrm{C}-\mathrm{H}$ bond functionalization, ${ }^{[167]}$ enamine phosphorylation, ${ }^{[168]}$ and so on. ${ }^{[169-171]}$ In most cases, they proposed that these reactions may undergo one or several single electron transfer (SET) processes (Scheme 76). However, the pathways of their photocatalytic reactions might involve ES-PCET or other PCET related processes, which is unique and interesting. Therefore, we propose further studies on the detailed mechanism of these coupling reactions with hydrogen evolution could be of great interests.

\section{Summary and outlook}

In summary, proton-coupled electron transfer has been widely applied in various aspects of organic synthesis, including the oxidation of single bond such as $\mathrm{C}-\mathrm{H}, \mathrm{N}-\mathrm{H}$, 
Examples of coupling reactions with hydrogen evolution: Enamine phosphorylation:

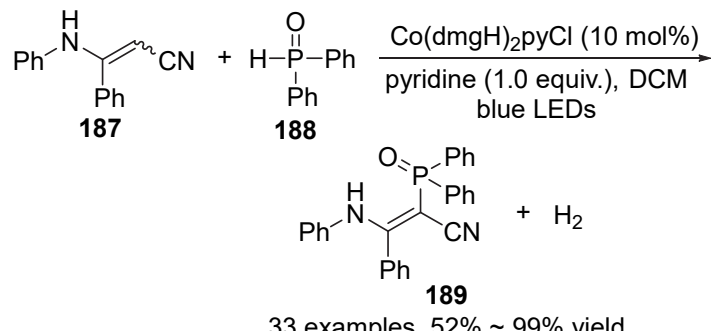

33 examples, $52 \% \sim 99 \%$ yield

$\mathrm{C}-\mathrm{H}$ bond functionalization:

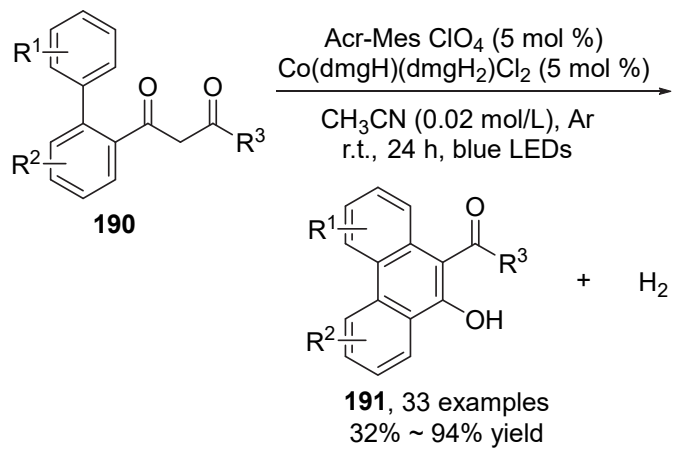

Scheme 75 Coupling reactions with hydrogen evolution

Proposed mechanism (for enamine phosphorylation):

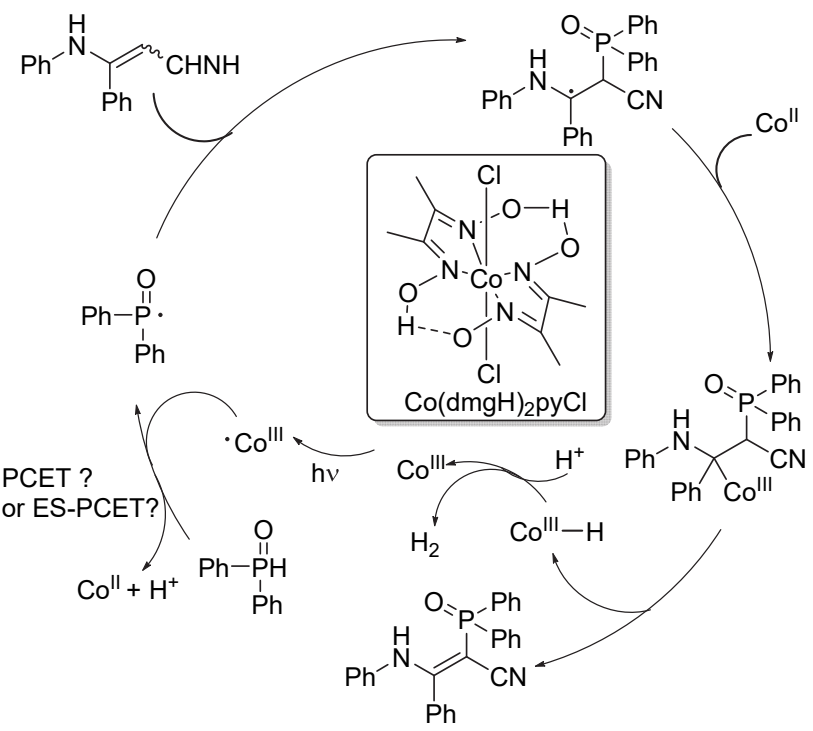

Scheme 76 Proposed mechanism of coupling reactions with hydrogen evolution which may involve ES-PCET

$\mathrm{O}-\mathrm{H}, \mathrm{P}-\mathrm{H}, \mathrm{S}-\mathrm{H}$ bonds, and the reduction of double bonds like $\mathrm{C}=\mathrm{C}, \mathrm{C}=\mathrm{N}, \mathrm{C}=\mathrm{O}$ bonds. Nowadays, new types of PCET like inner-sphere PCET reactions and excited-state PCET reactions have attracted the attentions from chemists of both experimental and theoretical backgrounds. We can forecast that the development of these new areas will flourish both experimentally and theoretically, and it should stimulate the application of PCET and also expand the frontiers of organic synthesis.

Compared with the blooming publications on the organic synthetic methodologies promoted by PCET, the professional and deep experimental studies on PCET mechanism for revealing its fundamental rules are relatively rare in China, ${ }^{[151,172-175]}$ however, we are glad to see that more publications on theoretical studies have been published from Chinese groups in last five year. ${ }^{[173-180]}$ Further explorations of PCET mechanism including how substrates and reaction conditions influence and regulate the PCET pathways, and so on, may provide novel ideas and methods for current synthetic methodology, and even help chemists discover unexpected patterns of chemical bond forming and breaking. Therefore, future works are supposed to focus on not only the synthetic applications, but also mechanism studies of PCET reactions, including new forms of PCET processes, which should benefit the development of other branches of chemistry.

\section{Acknowledgement}

This paper is dedicated to the $100^{\text {th }}$ anniversary of chemistry at Nankai University, and it is also dedicated to Prof Thomas J. Meyer at The University of North Carolina at Chapel Hill for his $80^{\text {th }}$ birthday. We are very grateful to the supports from State Key Laboratory of Elementoorganic Chemistry at Nankai University and Key Laboratory of Organofluorine Chemistry at Shanghai Institute of Organic Chemistry, Chinese Academy of Sciences. We appreciate the startup fund from Nankai University.

\section{References}

[1] Hammes-Schiffer, S.; Iordanova, N. Biochim. Biophys. Acta 2004, $1655,29$.

[2] Hammes-Schiffer, S. Acc. Chem. Res. 2009, 42, 1881.

[3] Mayer, J. M. Annu. Rev. Phys. Chem. 2004, 55, 363.

[4] Mayer, J. M.; Rhile, I. J. Biochim. Biophys. Acta 2004, 1655, 51.

[5] Rhile, I. J.; Markle, T. F.; Nagao, H.; DiPasquale, A. G.; Lam, O. P.; Lockwood, M. A.; Rotter, K.; Mayer, J. M. J. Am. Chem. Soc. 2006, 128,6075 .

[6] Tyburski, R.; Liu, T.; Glover, S. D.; Hammarström, L. J. Am. Chem. Soc. 2021, 143, 560.

[7] Gentry, E. C.; Knowles, R. R. Acc. Chem. Res. 2016, 49, 1546.

[8] Yang, J.-D.; Ji, P.; Xue, X.-S.; Cheng, J.-P. J. Am. Chem. Soc. 2018 140,8611

[9] Wu, X.; Zhu, C. Chin. J. Chem. 2019, 37, 171.

[10] Symes, M. D.; Surendranath, Y.; Lutterman, D. A.; Nocera, D. G. J. Am. Chem. Soc. 2011, 133, 5174.

[11] Guo, Y.; Liu, Y.; Qi, J.; Li, H.; He, L.; Lu, L.; Liu, C.; Gong, L.; Zhao, D.; Yang, Z. Acta Chim. Sinica 2017, 75, 914 (in Chinese). (郭宇, 刘瑜, 戚娟娟, 李慧, 赫兰兰, 卢丽男, 刘翠, 宫利东, 赵东霞, 杨忠志, 化学学报, 2017, 75, 914.)

[12] Odella, E.; Mora, S. J.; Wadsworth, B. L.; Huynh, M. T.; Goings, J. J.; Liddell, P. A.; Groy, T. L.; Gervaldo, M.; Sereno, L. E.; Gust, D.; Moore, T. A.; Moore, G. F.; Hammes-Schiffer, S.; Moore, A. L. J. Am. Chem. Soc. 2018, 140, 15450.

[13] Liu, T.; Guo, M.; Orthaber, A.; Lomoth, R.; Lundberg, M.; Ott, S.; Hammarström, L. Nat. Chem. 2018, 10, 881.

[14] Liu, T.; Tyburski, R.; Wang, S.; Fernandez-Teran, R.; Ott, S.; Hammarström, L. J. Am. Chem. Soc. 2019, 141, 17245.

[15] Bourrez, M.; Steinmetz, R.; Ott, S.; Gloaguen, F.; Hammarström, L. Nat. Chem. 2015, 7, 140 .

[16] Jackson, M. N.; Pegis, M. L.; Surendranath, Y. ACS Cent. Sci. 2019, 5,831 .

[17] Wenger, O. S. Chem.-Eur. J. 2011, 17, 11692. 
[18] Wenger, O. S. Acc. Chem. Res. 2013, 46, 1517.

[19] Chen, Z.; Wang, T.; Sun, T.; Chen, Z.; Sheng, T.; Hong, Y.-H.; Nan, Z.-A.; Zhu, J.; Zhou, Z.-Y.; Xia, H.; Sun, S.-G. Chin. J. Chem. 2018, 36, 1161 .

[20] Binstead, R. A.; Moyer, B. A.; Samuels, G. J.; Meyer, T. J. J. Am. Chem. Soc. 1981, 103, 2897.

[21] Liu, F.; Concepcion, J. J.; Jurss, J. W.; Cardolaccia, T.; Templeton, J. L.; Meyer, T. J. Inorg. Chem. 2008, 47, 1727.

[22] Bonin, J.; Robert, M. Photochem. Photobiol. 2011, 87, 1190.

[23] Hoffmann, N. Eur. J. Org. Chem. 2017, 2017, 1982.

[24] Hammes-Schiffer, S.; Soudackov, A. V. J. Phys. Chem. B 2008, 112, 14108.

[25] Sanderson, R. T. Polar Covalence, Academic Press, New York, 1983, p. 46.

[26] Sanderson, R. T. Chemical Bonds and Bond Energy, Academic Press, New York, 1976, p. 185.

[27] Marcus, R. A.; Sutin, N. Biochim. Biophys. Acta 1985, 811, 265.

[28] Bolton, J. R.; Archer, M. D. In Electron Transfer in Inorganic, Organic, and Biological Systems, American Chemical Society, Washington, 1991, pp. 7 23 .

[29] Marcus, R. A. J. Chem. Phys. 1956, 24, 966.

[30] Borgis, D.; Hynes, J. T. J. Phy. Chem. 1996, 100, 1118.

[31] Krishtalik, L. I. Biochim. Biophys. Acta 2000, 1458, 6.

[32] Soudackov, A.; Hammes-Schiffer, S. J. Chem. Phys. 1999, 111, 4672.

[33] Soudackov, A.; Hammes-Schiffer, S. J. Chem. Phys. 2000, 113, 2385.

[34] Hammes-Schiffer, S.; Stuchebrukhov, A. A. Chem. Rev. 2010, 110, 6939.

[35] Huynh, M. H. V.; Meyer, T. J. Chem. Rev. 2007, 107, 5004.

[36] Warren, J. J.; Tronic, T. A.; Mayer, J. M. Chem. Rev. 2010, 110, 6961.

[37] Weinberg, D. R.; Gagliardi, C. J.; Hull, J. F.; Murphy, C. F.; Kent, C. A.; Westlake, B. C.; Paul, A.; Ess, D. H.; McCafferty, D. G.; Meyer, T. J. Chem. Rev. 2012, 112, 4016.

[38] Waidmann, C. R.; Miller, A. J. M.; Ng, C.-W. A.; Scheuermann, M. L.; Porter, T. R.; Tronic, T. A.; Mayer, J. M. Energy Environ. Sci. 2012, 5, 7771 .

[39] Darcy, J. W.; Koronkiewicz, B.; Parada, G. A.; Mayer, J. M. Acc. Chem. Res. 2018, 51, 2391.

[40] Morton, C. M.; Zhu, Q.; Ripberger, H.; Troian-Gautier, L.; Toa, Z. S. D.; Knowles, R. R.; Alexanian, E. J. J. Am. Chem. Soc. 2019, $141,13253$.

[41] Tarantino, K. T.; Liu, P.; Knowles, R. R. J. Am. Chem. Soc. 2013, $135,10022$.

[42] Skone, J. H.; Soudackov, A. V.; Hammes-Schiffer, S. J. Am. Chem. Soc. 2006, 128, 16655.

[43] Liu, T.; Tyburski, R.; Wang, S.; Fernández-Terán, R.; Ott, S.; Hammarström, L. J. Am. Chem. Soc. 2019, 141, 17245.

[44] Huang, T.; Rountree, E. S.; Traywick, A. P.; Bayoumi, M.; Dempsey, J. L. J. Am. Chem. Soc. 2018, 140, 14655.

[45] Darcy, J. W.; Kolmar, S. S.; Mayer, J. M. J. Am. Chem. Soc. 2019, 141, 10777.

[46] Zhang, J.; Yang, J.-D.; Cheng, J.-P. Chem. Sci. 2020, 11, 3672.

[47] Chen, X.; Engle, K. M.; Wang, D. H.; Yu, J. Q. Angew. Chem., Int. Ed. 2009, 48, 5094.

[48] Giri, R.; Shi, B. F.; Engle, K. M.; Maugel, N.; Yu, J. Q. Chem. Soc. Rev. 2009, 38, 3242 .

[49] Engle, K. M.; Mei, T.-S.; Wasa, M.; Yu, J.-Q. Acc. Chem. Res. 2012, 45, 788 .

[50] Sun, C. L.; Li, B. J.; Shi, Z. J. Chem. Rev. 2011, 111, 1293

[51] Markle, T. F.; Darcy, J. W.; Mayer, J. M. Sci. Adv. 2018, 4, eaat5776.

[52] Sayfutyarova, E. R.; Goldsmith, Z. K.; Hammes-Schiffer, S. J. Am. Chem. Soc. 2018, 140, 15641.

[53] Sayfutyarova, E. R.; Lam, Y. C.; Hammes-Schiffer, S. J. Am. Chem. Soc. 2019, 141, 15183.

[54] Uraguchi, D.; Torii, M.; Ooi, T. ACS Catal. 2017, 7, 2765.
[55] Shevchenko, G. A.; Oppelaar, B.; List, B. Angew. Chem., Int. Ed. 2018, 57, 10756.

[56] Leng, L.; Ready, J. M. ACS Catal. 2020, 10, 13196.

[57] Wu, Z. J.; Xu, H. C. Angew. Chem., Int. Ed. 2017, 56, 4734

[58] Lennox, A. J. J.; Goes, S. L.; Webster, M. P.; Koolman, H. F.; Djuric, S. W.; Stahl, S. S. J. Am. Chem. Soc. 2018, 140, 11227.

[59] Zhang, Z.; Zhang, L.; Cao, Y.; Li, F.; Bai, G.; Liu, G.; Yang, Y.; Mo, F. Org. Lett. 2019, 21, 762 .

[60] Sharma, S.; Roy, A.; Shaw, K.; Bisai, A.; Paul, A. J. Org. Chem. 2020, $85,14926$.

[61] He, M. X.; Mo, Z. Y.; Wang, Z. Q.; Cheng, S. Y.; Xie, R. R.; Tang, H. T.; Pan, Y. M. Org. Lett. 2020, 22, 724

[62] Tanwar, L.; Borgel, J.; Ritter, T. J. Am. Chem. Soc. 2019, 141, 17983.

[63] Wang, Z.; Liu, Q.; Ji, X.; Deng, G.-J.; Huang, H. ACS Catal. 2019, 10,154 .

[64] Wu, Y.; Chen, J.-Y.; Ning, J.; Jiang, X.; Deng, J.; Deng, Y.; Xu, R.; He, W.-M. Green Chem. 2021, 23, 3950.

[65] Peng, S.; Lin, Y.; He, W.-M. Chin. J. Org. Chem. 2020, 40, 541 (in Chinese). (彭莎，林英武，何卫民，有机化学, 2020, 40, 541.)

[66] Luo, Y. R. Handbook of Bond Dissociation Energies in Organic Compounds, CRC Press, Boca Raton, 2003.

[67] Choi, G. J.; Knowles, R. R. J. Am. Chem. Soc. 2015, 137, 9226.

[68] Miller, D. C.; Choi, G. J.; Orbe, H. S.; Knowles, R. R. J. Am. Chem. Soc. 2015, 137, 13492.

[69] Tarantino, K. T.; Miller, D. C.; Callon, T. A.; Knowles, R. R. J. Am. Chem. Soc. 2015, 137, 6440.

[70] Choi, G. J.; Zhu, Q.; Miller, D. C.; Gu, C. J.; Knowles, R. R. Nature 2016, 539, 268.

[71] Gentry, E. C.; Rono, L. J.; Hale, M. E.; Matsuura, R.; Knowles, R. R. J. Am. Chem. Soc. 2018, 140, 3394.

[72] Zhu, Q.; Graff, D. E.; Knowles, R. R. J. Am. Chem. Soc. 2018, 140, 741.

[73] Nguyen, S. T.; Zhu, Q.; Knowles, R. R. ACS Catal. 2019, 9, 4502.

[74] Roos, C. B.; Demaerel, J.; Graff, D. E.; Knowles, R. R. J. Am. Chem. Soc. 2020, 142, 5974.

[75] Zhou, Z.; Li, Y.; Han, B.; Gong, L.; Meggers, E. Chem. Sci. 2017, 8,5757 .

[76] Yuan, W.; Zhou, Z.; Gong, L.; Meggers, E. Chem. Commun. 2017, 53,8964 .

[77] Jia, J.; Ho, Y. A.; Bulow, R. F.; Rueping, M. Chem.-Eur. J. 2018, 24,14054 .

[78] Moon, Y.; Jang, E.; Choi, S.; Hong, S. Org. Lett. 2018, 20, 240.

[79] Zheng, S.; Gutierrez-Bonet, A.; Molander, G. A. Chem 2019, 5, 339.

[80] Zhou, C.; Lei, T.; Wei, X. Z.; Ye, C.; Liu, Z.; Chen, B.; Tung, C. H.; Wu, L. Z. J. Am. Chem. Soc. 2020, 142, 16805.

[81] Zhu, L.; Xiong, P.; Mao, Z. Y.; Wang, Y. H.; Yan, X.; Lu, X.; Xu, H. C. Angew. Chem., Int. Ed. 2016, 55, 2226.

[82] Xiong, P.; Xu, H. H.; Xu, H. C. J. Am. Chem. Soc. 2017, 139, 2956.

[83] Hu, X.; Zhang, G.; Bu, F.; Nie, L.; Lei, A. ACS Catal. 2018, 8, 9370.

[84] Wang, F.; Gerken, J. B.; Bates, D. M.; Kim, Y. J.; Stahl, S. S. J. Am. Chem. Soc. 2020, 142, 12349.

[85] Xu, Z.; Huang, Z.; Li, Y.; Kuniyil, R.; Zhang, C.; Ackermann, L.; Ruan, Z. Green Chem. 2020, 22, 1099.

[86] Taylor, R. J. K.; Reid, M.; Foot, J.; Raw, S. A. Acc. Chem. Res. 2005, 38, 851 .

[87] Uyanik, M.; Ishihara, K. Chem. Commun. 2009, 2086.

[88] Ciriminna, R.; Pagliaro, M. Org. Process Res. Dev. 2010, 14 245-251.

[89] Hoover, J. M.; Stahl, S. S. J. Am. Chem. Soc. 2011, 133, 16901.

[90] Greene, J. F.; Hoover, J. M.; Mannel, D. S.; Root, T. W.; Stahl, S. S. Org. Process Res. Dev. 2013, 17, 1247.

[91] Rahimi, A.; Azarpira, A.; Kim, H.; Ralph, J.; Stahl, S. S. J. Am. Chem. Soc. 2013, 135, 6415.

[92] Steves, J. E.; Preger, Y.; Martinelli, J. R.; Welch, C. J.; Root, T. W.; Hawkins, J. M.; Stahl, S. S. Org. Process Res. Dev. 2015, 19, 1548. 
[93] Rafiee, M.; Konz, Z. M.; Graaf, M. D.; Koolman, H. F.; Stahl, S. S. ACS Catal. 2018, 8, 6738.

[94] Rafiee, M.; Alherech, M.; Karlen, S. D.; Stahl, S. S. J. Am. Chem. Soc. 2019, 141, 15266.

[95] Ota, E.; Wang, H.; Frye, N. L.; Knowles, R. R. J. Am. Chem. Soc. 2019, 141, 1457.

[96] Zhao, K.; Yamashita, K.; Carpenter, J. E.; Sherwood, T. C.; Ewing, W. R.; Cheng, P. T. W.; Knowles, R. R. J. Am. Chem. Soc. 2019, $141,8752$.

[97] Nguyen, S. T.; Murray, P. R. D.; Knowles, R. R. ACS Catal. 2019, 10,800 .

[98] Huang, L.; Ji, T.; Rueping, M. J. Am. Chem. Soc. 2020, 142, 3532.

[99] Tsui, E.; Metrano, A. J.; Tsuchiya, Y.; Knowles, R. R. Angew. Chem., Int. Ed. 2020, 59, 11845

[100] Tang, W.; Zhang, X. Chem. Rev. 2003, 103, 3029.

[101] Piou, T.; Rovis, T. Nature 2015, 527, 86.

[102] Bayeh, L.; Le, P. Q.; Tambar, U. K. Nature 2017, 547, 196.

[103] Koh, M. J.; Nguyen, T. T.; Lam, J. K.; Torker, S.; Hyvl, J.; Schrock, R. R.; Hoveyda, A. H. Nature 2017, 542, 80.

[104] Alonso, F.; Beletskaya, I. P.; Yus, M. Chem. Rev. 2004, 104, 3079.

[105] Xi, Y.; Dong, B.; McClain, E. J.; Wang, Q.; Gregg, T. L.; Akhmedov, N. G.; Petersen, J. L.; Shi, X. Angew. Chem., Int. Ed. 2014, 53, 4657.

[106] Hopkinson, M. N.; Tlahuext-Aca, A.; Glorius, F. Acc. Chem. Res. 2016, 49, 2261.

[107] Patel, M.; Saunthwal, R. K.; Verma, A. K. Acc. Chem. Res. 2017, $50,240$.

[108] Wang, J.; Zhang, S.; Xu, C.; Wojtas, L.; Akhmedov, N. G.; Chen, H.; Shi, X. Angew. Chem., Int. Ed. 2018, 57, 6915.

[109] Wang, H.; Li, Y.; Tang, Z.; Wang, S.; Zhang, H.; Cong, H.; Lei, A. ACS Catal. 2018, 8, 10599.

[110] Liu, Y.; Chen, X. L.; Li, X. Y.; Zhu, S. S.; Li, S. J.; Song, Y.; Qu, L. B.; Yu, B. J. Am. Chem. Soc. 2021, 143, 964.

[111] Kuss-Petermann, M.; Wenger, O. S. J. Phys. Chem. Lett. 2013, 4, 2535 .

[112] Griesbaum, K. Angew. Chem., Int. Ed. 1970, 9, 273.

[113] Heiba, E.-A. I.; Dessau, R. M. J. Org. Chem. 1967, 32, 3837.

[114] Huyser, E. S.; Kellogg, R. M. J. Org. Chem. 1966, 31, 3366.

[115] Zhao, R.; Lind, J.; Merenyi, G.; Eriksen, T. E. J. Am. Chem. Soc. 1994, 116, 12010 .

[116] Dang, H.-S.; Roberts, B. P. Tetrahedron Lett. 1999, 40, 8929.

[117] Fujisawa, H.; Hayakawa, Y.; Sasaki, Y.; Mukaiyama, T. Chem. Lett. 2001, 30, 632 .

[118] Benati, L.; Leardini, R.; Minozzi, M.; Nanni, D.; Scialpi, R.; Spagnolo, P.; Strazzari, S.; Zanardi, G. Angew. Chem., Int. Ed. 2004, 43, 3598 .

[119] Kemper, J.; Studer, A. Angew. Chem., Int. Ed. 2005, 44, 4914.

[120] Dénès, F.; Pichowicz, M.; Povie, G.; Renaud, P. Chem. Rev. 2014, 1142587.

[121] Qvortrup, K.; Rankic, D. A.; MacMillan, D. W. J. Am. Chem. Soc. 2014, 136, 626 .

[122] Hager, D.; MacMillan, D. W. J. Am. Chem. Soc. 2014, 136, 16986.

[123] Tyson, E. L.; Niemeyer, Z. L.; Yoon, T. P. J. Org. Chem. 2014, 79, 1427.

[124] He, W.-B.; Gao, L.-Q.; Chen, X.-J.; Wu, Z.-L.; Huang, Y.; Cao, Z.; Xu, X.-H.; He, W.-M. Chin. Chem. Lett. 2020, 31, 1895.

[125] Li, J.; Gu, Z.; Zhao, X.; Qiao, B.; Jiang, Z. Chem. Commun. 2019, $55,12916$.

[126] Liu, X.; Yin, Y.; Jiang, Z. Chem. Commun. 2019, 55, 11527.

[127] Yang, H.; Wei, G.; Jiang, Z. ACS Catal. 2019, 9, 9599.

[128] Zeng, G.; Li, Y.; Qiao, B.; Zhao, X.; Jiang, Z. Chem. Commun. 2019, 55, 11362 .

[129] Shi, J.; Wei, W. Chin. J. Org. Chem. 2020, 40, 2170 (in Chinese) (时建伟, 魏伟, 有机化学, 2020, 402170 .)

[130] Prasanna, R.; Guha, S.; Sekar, G. Org. Lett. 2019, 21, 2650.

[131] Kong, M.; Tan, Y.; Zhao, X.; Qiao, B.; Tan, C. H.; Cao, S.; Jiang, Z. J. Am. Chem. Soc. 2021, 143, 4024.

[132] Li, J.; He, L.; Liu, X.; Cheng, X.; Li, G. Angew. Chem., Int. Ed.
2019, 58, 1759.

[133] Zimmerman, H. E. Acc. Chem. Res. 2012, 45, 164.

[134] Benkeser, R. A.; Kaiser, E. M. J. Am. Chem. Soc. 1963, 85, 2858.

[135] Swenson, K. E.; Zemach, D.; Nanjundiah, C.; Kariv-Miller, E. J. Org. Chem. 1983, 48, 1777.

[136] Chaussard, J.; Combellas, C.; Thiebault, A. Tetrahedron Lett. 1987, $28,1173$.

[137] Zhou, F.; Jehoulet, C.; Bard, A. J. J. Am. Chem. Soc. 1992, 114, 11004

[138] Ishifune, M.; Yamashita, H.; Kera, Y.; Yamashita, N.; Hirata, K.; Murase, H.; Kashimura, S. Electrochim. Acta 2003, 48, 2405.

[139] Peters, B. K.; Rodriguez, K. X.; Reisberg, S. H.; Beil, S. B.; Hickey, D. P.; Kawamata, Y.; Collins, M.; Starr, J.; Chen, L.; Udyavara, S.; Klunder, K.; Gorey, T. J.; Anderson, S. L.; Neurock, M.; Minteer, S. D.; Baran, P. S. Science 2019, 363, 838.

[140] Qin, Y.; Lu, J.; Zou, Z.; Hong, H.; Li, Y.; Li, Y.; Chen, L.; Hu, J.; Huang, Y. Org. Chem. Front. 2020, 7, 1817.

[141] Liu, X.; Liu, R.; Qiu, J.; Cheng, X.; Li, G. Angew. Chem., Int. Ed. 2020, 59, 13962

[142] Rossolini, T.; Leitch, J. A.; Grainger, R.; Dixon, D. J. Org. Lett. 2018, 20, 6794.

[143] Vasu, D.; Fuentes de Arriba, A. L.; Leitch, J. A.; de Gombert, A.; Dixon, D. J. Chem. Sci. 2019, 10, 3401.

[144] Nicastri, M. C.; Lehnherr, D.; Lam, Y. H.; DiRocco, D. A.; Rovis, T. J. Am. Chem. Soc. 2020, 142, 987.

[145] Lehnherr, D.; Lam, Y. H.; Nicastri, M. C.; Liu, J.; Newman, J. A.; Regalado, E. L.; DiRocco, D. A.; Rovis, T. J. Am. Chem. Soc. 2020, 142, 468.

[146] Chen, M.; Zhao, X.; Yang, C.; Xia, W. Org. Lett. 2017, 19, 3807.

[147] Cao, K.; Tan, S. M.; Lee, R.; Yang, S.; Jia, H.; Zhao, X.; Qiao, B.; Jiang, Z. J. Am. Chem. Soc. 2019, 141, 5437.

[148] Huang, B.; Li, Y.; Yang, C.; Xia, W. Chem. Commun. 2019, 55, 6731.

[149] Nageswar Rao, D.; Rasheed, S.; Raina, G.; Ahmed, Q. N.; Jaladanki, C. K.; Bharatam, P. V.; Das, P. J. Org. Chem. 2017, 82, 7234.

[150] Zhang, G.; Fu, L.; Chen, P.; Zou, J.; Liu, G. Org. Lett. 2019, 21, 5015.

[151] Li, H; Zhang, M, T. Angew. Chem., Int. Ed. 2016, 55, 13132.

[152] Schrauben, J. N.; Cattaneo, M.; Day, T. C.; Tenderholt, A. L.; Mayer, J. M. J. Am. Chem. Soc. 2012, 134, 16635.

[153] Sirimanne, C. T.; Kerrigan, M. M.; Martin, P. D.; Kanjolia, R. K.; Elliott, S. D.; Winter, C. H. Inorg. Chem. 2015, 54, 7.

[154] Canteenwala, T.; Padmawar, P. A.; Chiang, L. Y. J. Am. Chem. Soc. 2005, 127,26

[155] Irebo, T.; Reece, S. Y.; Sjödin, M.; Nocera, D. G.; Hammarström, L. J. Am. Chem. Soc. 2007, 129, 15462.

[156] Pizano, A. A.; Yang, J. L.; Nocera, D. G. Chem. Sci. 2012, 3, 2457.

[157] Kuss-Petermann, M.; Wolf, H.; Stalke, D.; Wenger, O. S. J. Am. Chem. Soc. 2012, 134, 12844.

[158] Kuss-Petermann, M.; Wenger, O. S. J. Phys. Chem. A 2013, 117, 5726.

[159] Chen, J.; Kuss-Petermann, M.; Wenger, O. S. Chem.-Eur. J. 2014, 20, 4098.

[160] Bronner, C.; Wenger, O. S. Phys. Chem. Chem. Phys. 2014, 16 3617.

[161] Bowring, M. A.; Bradshaw, L. R.; Parada, G. A.; Pollock, T. P.; Fernández-Terán, R. J.; Kolmar, S. S.; Mercado, B. Q.; Schlenker, C. W.; Gamelin, D. R.; Mayer, J. M. J. Am. Chem. Soc. 2018, 140, 7449.

[162] Sayfutyarova, E. R.; Hammes-Schiffer, S. J. Phys. Chem. Lett. 2020, 11, 7109.

[163] Eisenhart, T. T.; Dempsey, J. L. J. Am. Chem. Soc. 2014, 136, 12221.

[164] Swords, W. B.; Meyer, G. J.; Hammarström, L. Chem. Sci. 2020, 11, 3460 .

[165] Lennox, J. C.; Kurtz, D. A.; Huang, T.; Dempsey, J. L. ACS Energy Lett. 2017, 2, 1246. 
[166] Parada, G. A.; Goldsmith, Z. K.; Kolmar, S.; Pettersson Rimgard, B.; Mercado, B. Q.; Hammarström, L.; Hammes-Schiffer, S.; Mayer, J. M. Science 2019, 364, 471.

[167] Guo, J. D.; Yang, X. L.; Chen, B.; Tung, C. H.; Wu, L. Z. Org. Lett. 2020, 22, 9627.

[168] Lei, T.; Liang, G.; Cheng, Y. Y.; Chen, B.; Tung, C. H.; Wu, L. Z. Org. Lett. 2020, 22, 5385.

[169] Wang, J. H.; Li, X. B.; Li, J.; Lei, T.; Wu, H. L.; Nan, X. L.; Tung, C. H.; Wu, L. Z. Chem. Commun. 2019, 55, 10376.

[170] Wang, J. H.; Lei, T.; Nan, X. L.; Wu, H. L.; Li, X. B.; Chen, B.; Tung, C. H.; Wu, L. Z. Org. Lett. 2019, 21, 5581.

[171] Huang, C.; Wang, J. H.; Qiao, J.; Fan, X. W.; Chen, B.; Tung, C. H.; Wu, L. Z. J. Org. Chem. 2019, 84, 12904.

[172] Li, H.; Guo, Y. H.; Wu, J. Y.; Zhang, M. T. Chem. Commun. 2019, 55,3465 .
[173] Chen, J.; Chen, J.; Liu, Y.; Zheng, Y.; Zhu, Q.; Han, G.; Shen, J.-R. J. Phys. Chem. Lett. 2019, 10, 3240.

[174] Ren, X.; Wang, X.; Sun, Y.; Chi, X.; Mangel, D.; Wang, H.; Sessler, J. L. Org. Chem. Front. 2019, 6, 584.

[175] Lin, Y.; Yan, Y.; Peng, W.; Qiao, X.; Huang, D.; Ji, H.; Chen, C.; Ma, W.; Zhao, J. J. Phys. Chem. Lett. 2020, 11, 3941.

[176] Zhang, X.; Ma, J.; Li, S.; Li, M.-D.; Guan, X.; Lan, X.; Zhu, R.; Phillips, D. L. J. Org. Chem. 2016, 81, 5330.

[177] Xu, Y.; Bao, P.; Song, K.; Shi, Q. J. Comput. Chem. 2019, 40, 1005.

[178] Zhong, W.; Wu, L.; Jiang, W.; Li, Y.; Mookan, N.; Liu, X. Dalton Trans. 2019, 48, 13711.

[179] Giret, Y.; Guo, P.; Wang, L.-F.; Cheng, J. J. Chem. Phys. 2020, 152, 124705.

[180] Song, K.; Shi, Q. J. Chem. Phys. 2017, 146, 184108. 